\title{
Biomimetic Synthesis Enables the Structure Revision of Littordials E and F,
}

and Drychampone B

Tomás Vieira de Castro, ${ }^{1}$ Oussama Yahiaoui, ${ }^{1}$ Ricardo A. Peralta, ${ }^{1}$ Thomas Fallon, ${ }^{1}$ Victor Lee, ${ }^{2}$ and Jonathan H. George*,1

${ }^{I}$ Department of Chemistry, University of Adelaide, Adelaide, SA 5005, Australia.

${ }^{2}$ Department of Chemistry, University of Oxford, Chemistry Research Laboratory, 12

Mansfield Road, Oxford, UK.

\section{Supporting Information}

\section{Table of Contents}

1. General methods 2

2. Experimental procedures 3

3. NMR spectra 11

4. Comparison of natural and synthetic NMR data 41

5. CD spectra 47

6. Single crystal X-ray data 48

7. Computational methods 51 


\section{General methods}

All chemicals were purchased from commercial suppliers and used as received. All reactions, unless otherwise stated, were performed under an inert atmosphere of $\mathrm{N}_{2}$. All organic extracts were dried over anhydrous magnesium sulfate. Thin layer chromatography was performed using aluminium sheets coated with silica gel $\mathrm{F}_{254}$. Visualization was aided by viewing under a UV lamp and staining with ceric ammonium molybdate or $\mathrm{KMnO}_{4}$ stain followed by heating. All $R_{f}$ values were measured to the nearest 0.05 . Flash column chromatography was performed using 40-63-micron grade silica gel. Melting points were recorded on a digital melting point apparatus and are uncorrected. Infrared spectra were recorded using an FT-IR spectrometer as the neat compounds. High field NMR spectra were recorded using either a $500 \mathrm{MHz}$ spectrometer $\left({ }^{1} \mathrm{H}\right.$ at $500 \mathrm{MHz},{ }^{13} \mathrm{C}$ at $\left.125 \mathrm{MHz}\right)$ or $600 \mathrm{MHz}$ spectrometer $\left({ }^{1} \mathrm{H}\right.$ at $600 \mathrm{MHz},{ }^{13} \mathrm{C}$ at $150 \mathrm{MHz}$ ). The solvent used for NMR spectra was $\mathrm{CDCl}_{3}$ unless otherwise specified. ${ }^{1} \mathrm{H}$ chemical shifts are reported in ppm on the $\delta$-scale relative to TMS $(\delta 0.0)$ or $\mathrm{CDCl}_{3}(\delta 7.26)$ and ${ }^{13} \mathrm{C}$ NMR chemical shifts are reported in ppm relative to $\mathrm{CDCl} 3(\delta 77.16)$. Multiplicities are reported as (br) broad, (s) singlet, (d) doublet, (t) triplet, (q) quartet, (quin) quintet, (sext) sextet, (hept) heptet and (m) multiplet. All $J$-values were rounded to the nearest $0.1 \mathrm{~Hz}$. ESI high resolution mass spectra were recorded on an ESI-TOF mass spectrometer. Circular dichroism (CD) spectra were measured using a JASCO J-715 spectropolarimeter. 


\section{Experimental procedures}
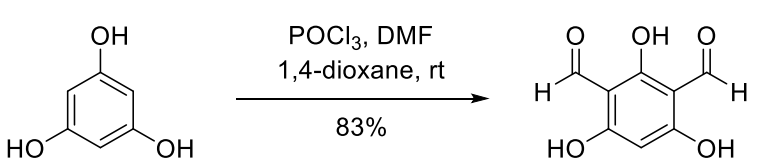

10

$\mathrm{POCl}_{3}(15.6 \mathrm{~mL}, 167 \mathrm{mmol})$ was added dropwise to DMF $(12.8 \mathrm{~mL}, 166 \mathrm{mmol})$ with vigorous stirring. The solution was stirred under a $\mathrm{N}_{2}$ atmosphere at room temperature for $30 \mathrm{~min}$. This Vilsmeier reagent was transferred dropwise, via cannula, to a stirred solution of anhydrous phloroglucinol (10.1 g, $79.9 \mathrm{mmol})$ in 1,4-dioxane $(50 \mathrm{~mL})$ under a $\mathrm{N}_{2}$ atmosphere. This mixture was vigorously stirred at room temperature for $16 \mathrm{~h}$. The resulting yellow solid was cooled to $0{ }^{\circ} \mathrm{C}$ and subsequently added to an ice-water slurry (approx. $400 \mathrm{~mL}$ ). The mixture was slowly warmed to room temperature and stirred for a further $4 \mathrm{~h}$. The resulting orange precipitate was filtered and washed with cold $\mathrm{H}_{2} \mathrm{O}$. This solid was dissolved in $\mathrm{H}_{2} \mathrm{O}(50 \mathrm{~mL})$, heated under reflux for $5 \mathrm{~min}$ and then cooled to $0{ }^{\circ} \mathrm{C}$. The resulting solid was filtered, washed with cold $\mathrm{H}_{2} \mathrm{O}$ and dried in an oven at $110{ }^{\circ} \mathrm{C}$ to constant weight to yield diformylphloroglucinol (10) as a red-orange powder (12.1 g, $66.4 \mathrm{mmol}, 83 \%)$. Data for 10 matched that of published literature. ${ }^{1}$

Rf: 0.15 (petrol/EtOAc, 1:1)

IR (neat): 2887, 1598, 1502, 1438, 1393, 1253, 1187, 1096, 947, $846 \mathrm{~cm}^{-1}$

${ }^{1}$ H NMR (500 MHz, d6-DMSO): $\delta 12.56$ (br s, 2H), 10.01 (s, 2H), 5.89 (s, 1H)

${ }^{13}$ C NMR (125 MHz, d6-DMSO): $\delta$ 191.4, 169.5, 169.2, 103.8, 93.9

\footnotetext{
${ }^{1}$ Dittmer, C.; Rabbe, G.; Hintermann, L. Eur. J. Org. Chem. 2007, 5886.
} 


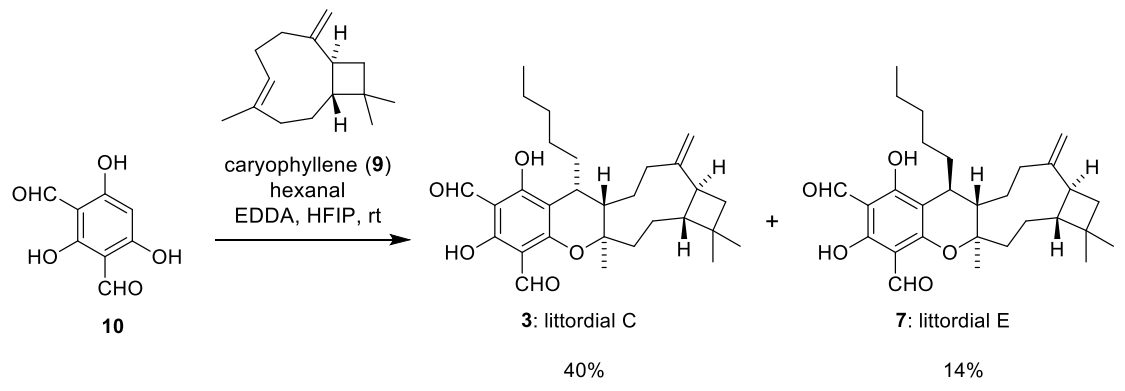

To a round-bottom flask open to air was added (-)-caryophyllene (9) (1.52 mL, $7.25 \mathrm{mmol})$, HFIP $(20 \mathrm{~mL})$, and hexanal $(0.85 \mathrm{~mL}, 6.81 \mathrm{mmol})$. To this biphasic mixture was added 10 (400 $\mathrm{mg}, 2.20 \mathrm{mmol}$ ). The reaction mixture was stirred vigorously for $5 \mathrm{~min}$ after which, EDDA (60 $\mathrm{mg}, 0.33 \mathrm{mmol}$ ) was added in a single portion. The mixture was stirred at room temperature for $4 \mathrm{~h}$. The solvent was removed under reduced pressure. Excess hexanal was removed on the rotary evaporator and on a high vacuum pump. Unreacted caryophyllene (9) was removed by eluting the reaction mixture through a pad of $\mathrm{SiO}_{2}$ using neat petrol as the eluent. Trituration of the resulting mixture with diethyl ether yielded a mixture of diastereomers as an orange gum. These diastereomers were separated by flash chromatography using a Biotage Isolera ${ }^{\mathrm{TM}}$ One Flash Chromatography System with iLOK ${ }^{\mathrm{TM}}$ Empty Solid Load Cartridges, and Carl Roth silica gel 60 (230 - 400 mesh grade), and 1\% $\mathrm{Et}_{2} \mathrm{O} /$ petrol as eluent, to give 3 (400 mg, 0.85 $\mathrm{mmol}, 40 \%)$ as a yellow powder and $7(139 \mathrm{mg}, 0.30 \mathrm{mmol}, 14 \%)$ as a yellow oil. To obtain single crystals suitable for X-ray analysis, littordial C (20 mg, $43 \mu \mathrm{mol})$ was dissolved in ethanol $(0.5 \mathrm{~mL})$ in a $5 \mathrm{~mL}$ vial and left at room temperature overnight to afford colourless prisms $(10 \mathrm{mg}, 50 \%)$.

\section{Data for 3:}

Mp: $105-108{ }^{\circ} \mathrm{C}$

R: 0.65 (5\% EtOAc/petrol)

$[\boldsymbol{\alpha}]_{D}^{25}=-50.4\left(\mathrm{c} 1.0, \mathrm{CH}_{2} \mathrm{Cl}_{2}\right)$, c.f. $[\boldsymbol{\alpha}]_{D}^{25}=+36.9\left(\mathrm{c} 0.1, \mathrm{CH}_{2} \mathrm{Cl}_{2}\right)$ from Xu et al.

IR (neat): 2951, 1633, 1441, 1382, 1304, 1178, $890 \mathrm{~cm}^{-1}$

${ }^{1}$ H NMR (600 MHz, CDCl $)$ ): $\boldsymbol{\delta} 13.25$ (s, 1H), 13.06 (s, 1H), 4.92 (s, 1H), 4.90 (s, 1H), 2.93 $(\mathrm{m}, 1 \mathrm{H}), 2.41(\mathrm{q}, J=8.7 \mathrm{~Hz}, 1 \mathrm{H}), 2.32(\mathrm{td}, J=13.1,11.9,3.2 \mathrm{~Hz}, 1 \mathrm{H}), 2.19(\mathrm{dd}, J=16.8,3.9$ $\mathrm{Hz}, 1 \mathrm{H}), 2.02$ (dd, $J=12.6,9.2 \mathrm{~Hz}, 1 \mathrm{H}$ ), $1.77-1.65$ (overlapped, m, 5H), $1.61-1.53$ (overlapped, m, 2H), 1.46 (s, 3H), $1.39-1.35$ (overlapped, m, 3H), 1.31 - 1.22 (overlapped, m, 8H), 1.17 (overlapped, m, 1H), 0.97 (s, 3H), $0.92(\mathrm{~s}, 3 \mathrm{H}), 0.85$ (t, $J=7.1 \mathrm{~Hz}, 3 \mathrm{H})$

${ }^{13}$ C NMR (150 MHz, CDCl $)$ : $\boldsymbol{\delta}$ 192.2, 192.0, 168.0, 167.5, 164.0, 150.9, 111.3, 111.2, 105.8, $104.3,88.2,59.5,47.8,42.5,40.7,36.1,35.6,34.8,32.3,29.7,29.6,29.5,28.6,24.8,23.6$, 23.3, 22.8, 21.8, 14.2

HRMS (ESI) m/z: $[\mathrm{M}+\mathrm{H}]^{+}$Calculated for $\mathrm{C}_{29} \mathrm{H}_{42} \mathrm{O}_{5} 469.2949$; found 469.2955

\section{Data for 7:}

$\mathbf{R}_{\mathbf{f}}: 0.60$ (5\% EtOAc/petrol)

$[\alpha]_{D}^{25}=+45.1\left(\right.$ c .7, $\left.\mathrm{CH}_{2} \mathrm{Cl}_{2}\right)$, c.f. $[\boldsymbol{\alpha}]_{D}^{25}=-16.7\left(\mathrm{c} 0.1, \mathrm{CH}_{2} \mathrm{Cl}_{2}\right)$ from Xu et al. 
IR (neat): 2953, 2926, 1631, 1440, 1382, 1306, 1177, $855 \mathrm{~cm}^{-1}$

${ }^{1}$ H NMR (600 MHz, CDCl $\left.\mathbf{~}_{3}\right): \delta 13.42$ (s, 1H), 13.33 (s, 1H), 4.94 (s, 1H), 4.90 (s, 1H), 2.51 (dt, $J=13.9,5.2 \mathrm{~Hz}, 1 \mathrm{H}), 2.46$ (ddd, $J=9.2,5.0,2.6 \mathrm{~Hz}, 1 \mathrm{H}), 2.43-2.38(\mathrm{~m}, 1 \mathrm{H}), 2.24-2.06$ (overlapped, m, 4H), 2.01 (ddd, $J=11.8,10.1,5.5 \mathrm{~Hz}, 1 \mathrm{H}$ ), 1.95 (ddd, $J=15.4,5.6,3.1 \mathrm{~Hz}$, $1 \mathrm{H}), 1.84$ (dddd, $J=15.1,12.6,5.5,3.1 \mathrm{~Hz}, 1 \mathrm{H}$ ), 1.71 (overlapped, m, 2H), 1.63 (dd, $J=10.7$, $7.7 \mathrm{~Hz}, 1 \mathrm{H}), 1.59-1.53(\mathrm{~m}, 1 \mathrm{H}), 1.48-1.37$ (overlapped, m, 2H), $1.14(\mathrm{~m}, 1 \mathrm{H}), 1.12(\mathrm{~s}, 3 \mathrm{H})$, 1.02 (overlapped, m, 1H), $1.01(\mathrm{~s}, 3 \mathrm{H}), 1.00(\mathrm{~s}, 3 \mathrm{H}), 0.84$ (t, J=7.1 Hz, 4H), 0.77 (m, 1H).

${ }^{13} \mathbf{C}$ NMR (150 MHz, $\left.\mathbf{C D C l}_{3}\right): \boldsymbol{\delta} 192.4,192.1,169.1,168.0,164.6,152.3,110.5,106.0,105.0$, 104.2 , 84.0, 53.4, 42.8, 38.8, 37.9, 36.4, 35.7, 35.1, 33.8, 33.4, 32.3, 30.7, 29.0, 24.6, 22.8, $22.7,22.3,21.6,14.3$

HRMS (ESI) m/z: $[\mathrm{M}+\mathrm{H}]^{+}$Calculated for $\mathrm{C}_{29} \mathrm{H}_{42} \mathrm{O}_{5} 469.2949$; found 469.2936 


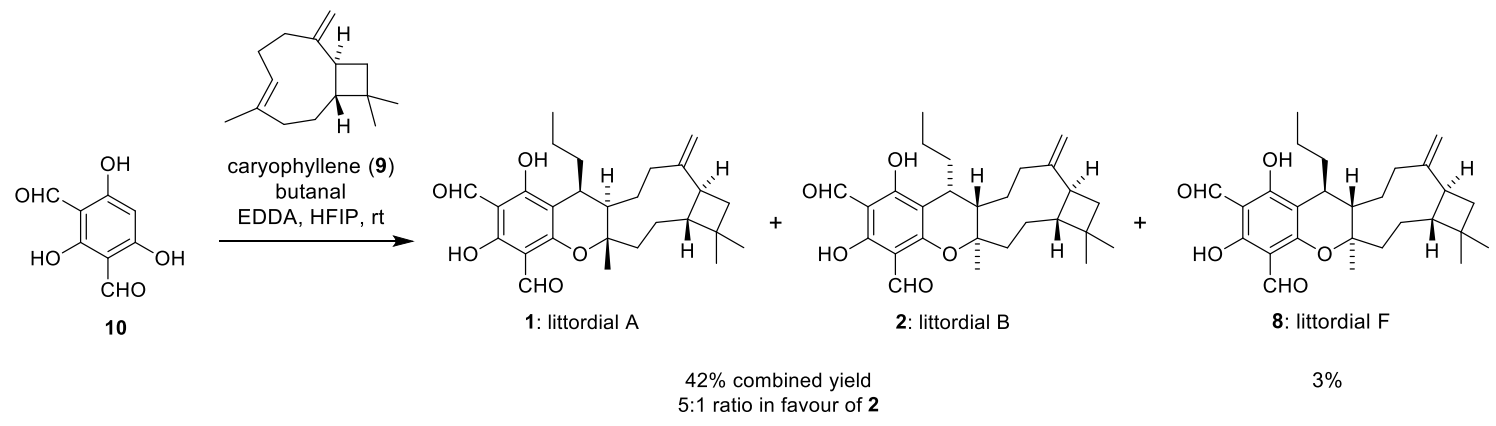

To a round-bottom flask open to air, was added (-)-caryophyllene $(9)(1.73 \mathrm{~mL}, 8.23 \mathrm{mmol})$, HFIP $(25 \mathrm{~mL})$, and butanal $(0.75 \mathrm{~mL}, 8.23 \mathrm{mmol})$. To this biphasic mixture was added 10 (500 $\mathrm{mg}, 2.75 \mathrm{mmol})$. The reaction mixture was stirred vigorously for $5 \mathrm{~min}$ after which EDDA (99.0 $\mathrm{mg}, 0.55 \mathrm{mmol}$ ) was added in a single portion. The mixture was stirred at room temperature for $4 \mathrm{~h}$. The solvent was removed under reduced pressure. Excess butanal was removed on the rotary evaporator and on a high vacuum pump. Unreacted caryophyllene (9) was removed by eluting the reaction mixture through a pad of $\mathrm{SiO}_{2}$ using petrol. Trituration of the resulting mixture with diethyl ether yielded a mixture of diastereomers as an orange gum. These compounds were separated by flash chromatography using a Biotage Isolera ${ }^{\mathrm{TM}}$ One Flash Chromatography System with iLOK ${ }^{\mathrm{TM}}$ Empty Solid Load Cartridges, and Carl Roth silica gel 60 (230 - 400 mesh grade), and with $1 \% \mathrm{Et}_{2} \mathrm{O} /$ petrol as eluent, to give 8 (40.8 mg, $0.09 \mathrm{mmol}, 3 \%$ ) as a pale yellow oil and an inseparable mixture of $\mathbf{1}$ and $\mathbf{2}$ (5:1 ratio in favour of littordial B, $492 \mathrm{mg}, 1.15 \mathrm{mmol}, 42 \%$ ) as a yellow powder.

\section{Data for 8:}

$\mathbf{R}_{\mathbf{f}}: 0.60$ (5\% EtOAc/petrol)

$[\boldsymbol{\alpha}]_{\boldsymbol{D}}^{\mathbf{2 5}}=+44.4\left(\mathrm{c} 0.62, \mathrm{CH}_{2} \mathrm{Cl}_{2}\right)$, c.f. $[\boldsymbol{\alpha}]_{D}^{25}=-42.0\left(\mathrm{c} 0.2, \mathrm{CH}_{2} \mathrm{Cl}_{2}\right)$ from Zhu et al.

IR (neat): 2954, 2929, 1631, 1441, 1383, 1306, 1178, $846 \mathrm{~cm}^{-1}$

${ }^{1}$ H NMR (600 MHz, CDCl 3 ): $\boldsymbol{\delta} 13.42$ (s, 1H), 13.32 (s, 1H), 10.16 (s, 1H), $10.01(\mathrm{~s}, 1 \mathrm{H}), 4.95$ (s, 1H), $4.90(\mathrm{~s}, 1 \mathrm{H}), 2.51(\mathrm{dt}, J=13.9,5.4,1 \mathrm{H}), 2.47(\mathrm{~m}, 1 \mathrm{H}), 2.41(\mathrm{~m}, 1 \mathrm{H}), 2.22-2.10$ (overlapped, 4H), 1.99 (m, 1H), 1.95 (ddd, $J=15.4,5.7,3.0 \mathrm{~Hz}, 2 \mathrm{H}), 1.84$ (dddd, $J=15.1$, 12.2, 5.3, 3.0 Hz, 1H), $1.72-1.69$ (overlapped, m, 2H), 1.63 (dd, $J=10.7,7.7 \mathrm{~Hz}, 1 \mathrm{H}), 1.57$ (m, 1H), $1.43(\mathrm{~m}$, overlapped, 1H), $1.12(\mathrm{~s}, 3 \mathrm{H}), 1.04(\mathrm{~m}, 1 \mathrm{H}), 1.01(\mathrm{~s}, 3 \mathrm{H}), 1.00(\mathrm{~s}, 3 \mathrm{H}), 0.83$ (overlapped, $\mathrm{m}, 1 \mathrm{H}$ ), 0.81 (overlapped, $\mathrm{m}, 3 \mathrm{H}$ )

${ }^{13} \mathbf{C}$ NMR (150 MHz, $\left.\mathbf{C D C l}_{3}\right): \delta$ 192.3, 192.1, 169.1, 168.0, 164.6, 152.4, 110.5, 106.1, 105.0, 104.2 , 84.1, 53.6, 42.7, 39.0, 38.1, 36.4, 35.8, 35.2, 33.8, 33.2, 31.7, 30.7, 22.7, 22.3, 21.5, $18.4,14.6$

HRMS (ESI) m/z: $[\mathrm{M}+\mathrm{H}]^{+}$Calculated for $\mathrm{C}_{27} \mathrm{H}_{38} \mathrm{O}_{5} 441.2636$; found 441.2640 


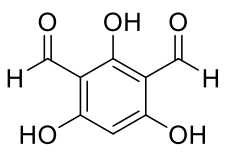

10
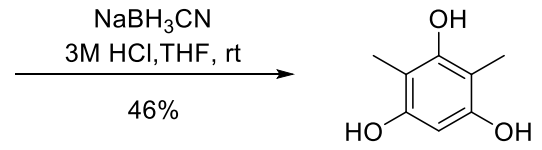

21

To a stirred solution of $\mathbf{1 0}(5.47 \mathrm{~g}, 30.0 \mathrm{mmol})$ and $\mathrm{NaBH}_{3} \mathrm{CN}(9.42 \mathrm{~g}, 150 \mathrm{mmol})$ in THF (85 $\mathrm{mL})$ was added $3 \mathrm{M} \mathrm{HCl}(50 \mathrm{~mL}, 150 \mathrm{mmol})$ dropwise. The reaction mixture was stirred at room temperature for $3 \mathrm{~h}$. Upon completion, the mixture was diluted with $\mathrm{H}_{2} \mathrm{O}$ and extracted with EtOAc ( $3 \times 150 \mathrm{~mL})$. The combined organic extracts were washed with $\mathrm{H}_{2} \mathrm{O}(150 \mathrm{~mL})$, brine $(2 \times 150 \mathrm{~mL})$, dried over anhydrous $\mathrm{MgSO}_{4}$, filtered and concentrated in vacuo. The crude residue was purified by flash column chromatography on $\mathrm{SiO}_{2}$ (petrol/EtOAc, 5:1) to afford 21 (2.12 g, $13.8 \mathrm{mmol}, 46 \%)$ as a pale orange solid. Data for 21 matched that of published literature. ${ }^{2}$

Mp: $158.6-161.0^{\circ} \mathrm{C}$

$\mathbf{R}_{\mathbf{f}}: 0.50$ (petrol/EtOAc, $1: 1$ )

IR (neat): 3525, 3423, 2942, 1611, 1511, 1460, 1280, 1249, $1150 \mathrm{~cm}^{-1}$

${ }^{1}$ H NMR (500 MHz, CD 3 OD): $\delta 5.97$ (s, 1H), 2.00 (s, 6H)

${ }^{13}$ C NMR (125 MHz, CD ${ }_{3}$ OD): $\delta$ 154.3, 104.2, 96.1, 8.5

\footnotetext{
${ }^{2}$ Jung, J. -W.; Damodar, K.; Kim, J. -K.; Jun, J. -G. Chin. Chem. Lett. 2017, 28, 1114.
} 


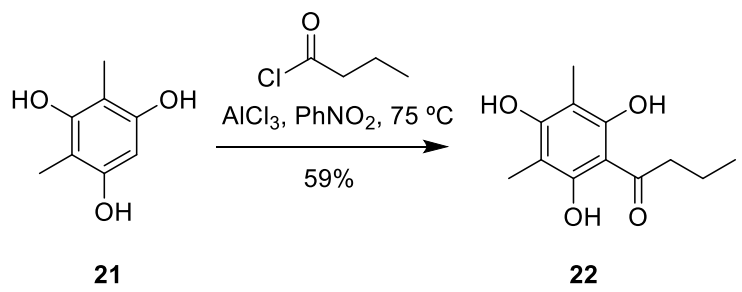

To a stirred of solution of $21(258 \mathrm{mg}, 1.67 \mathrm{mmol})$ in $\mathrm{PhNO}_{2}(6 \mathrm{~mL})$ at $0{ }^{\circ} \mathrm{C}$, was added $\mathrm{AlCl}_{3}$ $(885 \mathrm{mg}, 6.64 \mathrm{mmol})$. This mixture was stirred at $0{ }^{\circ} \mathrm{C}$ for $10 \mathrm{~min}$. Butyryl chloride $(0.19 \mathrm{~mL}$, $1.83 \mathrm{mmol}$ ) was added dropwise to the reaction which was then warmed to $75^{\circ} \mathrm{C}$ using an aluminium heating block and stirred for $3.5 \mathrm{~h}$. The resulting dark red solution was cooled to room temperature and diluted with cold water. This mixture was extracted with EtOAc $(3 \times 20$ $\mathrm{mL})$. The combined organic phases were extracted with $1 \mathrm{M} \mathrm{NaOH}(2 \times 15 \mathrm{~mL})$. The aqueous extracts were acidified by conc. $\mathrm{HCl}$. The aqueous layer was extracted with EtOAc $(3 \times 20$ $\mathrm{mL}$ ). The combined organic extracts were dried over $\mathrm{MgSO}_{4}$, filtered, and concentrated in vасио. The crude residue was purified by flash column chromatography on $\mathrm{SiO}_{2}$ (pet/EtOAc, 10:1 $\rightarrow$ 4:1 gradient elution) to afford $22(223 \mathrm{mg}, 0.99 \mathrm{mmol}, 59 \%)$ as a pale orange solid. Data for 22 matched that of published literature. ${ }^{3}$

Mp: $136.4-138.6{ }^{\circ} \mathrm{C}$

Rf: 0.30 (petrol/EtOAc, $4: 1$ )

IR (neat): 3468, 2964, 2875, 1612, 1432, 1379, 1320, 1284, 1238, 1199, $1159 \mathrm{~cm}^{-1}$

${ }^{1}$ H NMR (500 MHz, d6-DMSO): $\boldsymbol{\delta} 11.49$ (s, 2H), 9.20 (s, 1H), 3.04 (t, $\left.J=7.3 \mathrm{~Hz}, 2 \mathrm{H}\right), 1.96$ (s, 6H), $1.62(\mathrm{~h}, J=7.3 \mathrm{~Hz}, 1 \mathrm{H}), 0.91$ (t, $J=7.3 \mathrm{~Hz}, 3 \mathrm{H})$

${ }^{13}$ C NMR (125 MHz, d6-DMSO): $\boldsymbol{\delta}$ 206.0, 160.3, 158.4, 105.3, 102.6, 45.5, 18.0, 14.0, 8.6

HRMS (ESI) m/z: $[\mathrm{M}+\mathrm{H}]^{+}$Calculated for $\mathrm{C}_{12} \mathrm{H}_{17} \mathrm{O}_{4} 225.1121$; found 225.1116

\footnotetext{
${ }^{3}$ Wu, J.; Mu, R.; Sun, M.; Zhao, N.; Pan, M.; Li, H.; Dong, Y.; Sun, Z.; Bai, J.; Hu, M.; Nathan, C. F.; Javid, B.; Liu, G. ACS Infect. Dis. 2019, 5, 1087.
} 


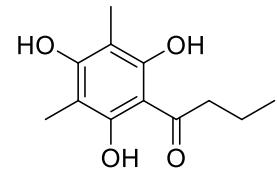

22
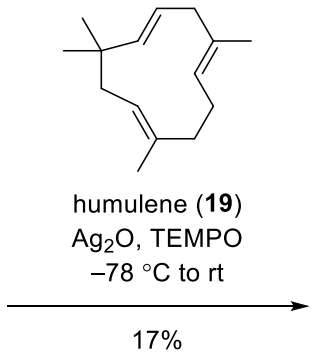

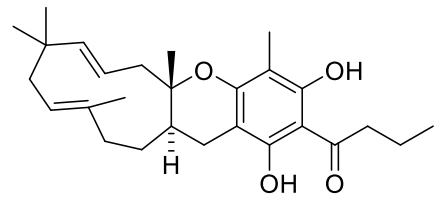

18: drychampone B

To a solution of $22(53.8 \mathrm{mg}, 0.24 \mathrm{mmol})$ and humulene $(19)(0.07 \mathrm{~mL}, 0.30 \mathrm{mmol})$ in $\mathrm{Et}_{2} \mathrm{O}(5$ $\mathrm{mL})$ at $-78{ }^{\circ} \mathrm{C}$ in a cooling bath of dry ice and acetone was added $\mathrm{Ag}_{2} \mathrm{O}(69 \mathrm{mg}, 0.30 \mathrm{mmol})$ and TEMPO $(78.3 \mathrm{mg}, 0.50 \mathrm{mmol})$. The reaction was stirred at $-78{ }^{\circ} \mathrm{C}$ for $1 \mathrm{~h}$, then slowly warmed to room temperature where it was stirred for a further $16 \mathrm{~h}$. The mixture was filtered and concentrated in vacuo. The crude residue was purified by flash column chromatography on $\mathrm{SiO}_{2}(1: 0 \rightarrow 30: 1$ petrol/EtOAc gradient elution) to yield 18 as a yellow gum (18.8 mg, 0.04 mmol, 17\%).

$\mathbf{R}_{\mathbf{f}}: 0.65$ (petrol/EtOAc, $5: 1$ )

IR (neat): 3520, 3422, 2932, 1610, 1421, 1379, 1202, 1159, $1106 \mathrm{~cm}^{-1}$

${ }^{1} \mathbf{H}$ NMR (600 MHz, CDCl $)$ ): $\boldsymbol{\delta} 5.18-5.14(\mathrm{~m}, 2 \mathrm{H}), 5.03(\mathrm{~m}, 1 \mathrm{H}), 3.00(\mathrm{td}, J=7.4,3.8 \mathrm{~Hz}$, 2H), $2.92(\mathrm{dd}, J=16.1,5.7 \mathrm{~Hz}, 1 \mathrm{H}), 2.60(\mathrm{~d}, J=14.5 \mathrm{~Hz}, 1 \mathrm{H}), 2.37$ (ddd, $J=14.5,7.1,3.0$ $\mathrm{Hz}, 1 \mathrm{H}), 2.19(\mathrm{t}, J=12.4 \mathrm{~Hz}, 1 \mathrm{H}), 2.16-2.09(\mathrm{~m}, 1 \mathrm{H}), 2.07$ (overlapped, 4H), $1.89-1.82$ (m, 2H), $1.77(\mathrm{dd}, J=12.9,4.4 \mathrm{~Hz}, 1 \mathrm{H}), 1.70(\mathrm{q}, J=7.4 \mathrm{~Hz}, 2 \mathrm{H}), 1.63(\mathrm{~s}, 3 \mathrm{H}), 1.34(\mathrm{~m}, 1 \mathrm{H}), 1.21$ $-1.15(\mathrm{~m}, 1 \mathrm{H}), 1.14(\mathrm{~s}, 3 \mathrm{H}), 1.06(\mathrm{~s}, 3 \mathrm{H}), 1.02(\mathrm{~s}, 3 \mathrm{H}), 0.97(\mathrm{t}, J=7.4 \mathrm{~Hz}, 3 \mathrm{H})$

${ }^{13}$ C NMR (150 MHz, $\left.\mathbf{C D C l}_{3}\right): \boldsymbol{\delta} 206.4,162.8,157.5,154.7,143.1,136.7,123.2,120.2,105.8$, $101.5,99.5,82.2,46.7,42.9,41.5,38.4,37.9,34.8,30.5,30.4,24.4,23.9,20.1,19.0,17.3$, $14.2,7.2$

HRMS (ESI) m/z: $[\mathrm{M}+\mathrm{H}]^{+}$Calculated for $\mathrm{C}_{27} \mathrm{H}_{40} \mathrm{O}_{4} 427.2843$; found 427.2837 
Optimization of the biomimetic synthesis of drychampone B:

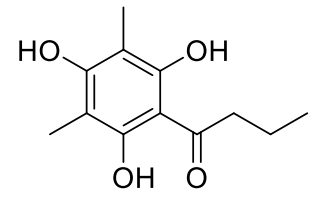

22

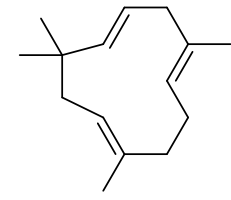

humulene (19)

Oxidant

Conditions

\begin{tabular}{|c|c|c|c|c|c|}
\hline Entry & $\begin{array}{c}\text { Humulene } \\
\text { equivalents }\end{array}$ & Oxidants (eq) & Solvent & Conditions & Result \\
\hline 1 & 1.2 & $\begin{array}{c}\mathrm{Ag}_{2} \mathrm{O}(1.2) \\
\operatorname{TEMPO}(2.0)\end{array}$ & $\mathrm{Et}_{2} \mathrm{O}$ & $-78^{\circ} \mathrm{C} \rightarrow \mathrm{rt}, 18 \mathrm{~h}$ & $17 \%$ \\
\hline 2 & 2.0 & $\begin{array}{c}\mathrm{Ag}_{2} \mathrm{O}(1.2) \\
\text { TEMPO (2.0) }\end{array}$ & $\mathrm{Et}_{2} \mathrm{O}$ & $-78^{\circ} \mathrm{C} \rightarrow \mathrm{rt}, 17 \mathrm{~h}$ & $15 \%$ \\
\hline 3 & 2.0 & $\begin{array}{c}\mathrm{Ag}_{2} \mathrm{O}(1.2) \\
\text { TEMPO (0.4) }\end{array}$ & $\mathrm{Et}_{2} \mathrm{O}$ & $-78^{\circ} \mathrm{C} \rightarrow \mathrm{rt}, 17 \mathrm{~h}$ & No reaction \\
\hline 4 & 2.0 & $\begin{array}{c}\mathrm{Ag}_{2} \mathrm{O}(1.2) \\
\operatorname{TEMPO}(1.1)\end{array}$ & $\mathrm{Et}_{2} \mathrm{O}$ & $-78^{\circ} \mathrm{C} \rightarrow \mathrm{rt}, 17 \mathrm{~h}$ & No reaction \\
\hline 5 & 2.0 & $\mathrm{Ag}_{2} \mathrm{O}(1.2)$ & Benzene & $\mathrm{rt}, 17 \mathrm{~h}$ & No reaction \\
\hline 6 & 2.0 & $\begin{array}{c}\text { PIDA (1.2) } \\
\text { TEMPO (2.0) }\end{array}$ & $\mathrm{Et}_{2} \mathrm{O}$ & $-78^{\circ} \mathrm{C} \rightarrow \mathrm{rt}, 4 \mathrm{~h}$ & Decomposition \\
\hline 7 & 2.0 & $\begin{array}{c}\text { PIFA (1.2) } \\
\text { TEMPO (2.0) }\end{array}$ & THF & $-78^{\circ} \mathrm{C} \rightarrow \mathrm{rt}, 2 \mathrm{~h}$ & Decomposition \\
\hline 8 & 2.0 & $\begin{array}{c}\text { CAN (1.2) } \\
\text { TEMPO (2.0) }\end{array}$ & $\mathrm{MeOH}$ & $-78^{\circ} \mathrm{C} \rightarrow \mathrm{rt}, 3 \mathrm{~h}$ & Decomposition \\
\hline 9 & 2.0 & $\begin{array}{c}\text { CAN (1.2) } \\
\text { TEMPO (1.2) }\end{array}$ & $\mathrm{MeOH}$ & $-78^{\circ} \mathrm{C} \rightarrow \mathrm{rt}, 3 \mathrm{~h}$ & Decomposition \\
\hline 10 & 2.0 & $\mathrm{MnO}_{2}(2.2)$ & Benzene & $\mathrm{rt}, 24 \mathrm{~h}$ & No reaction \\
\hline 11 & 2.0 & TEMPO (2.0) & $\mathrm{Et}_{2} \mathrm{O}$ & Air, rt, $5 \mathrm{~d}$ & No reaction \\
\hline
\end{tabular}


3. NMR Spectra
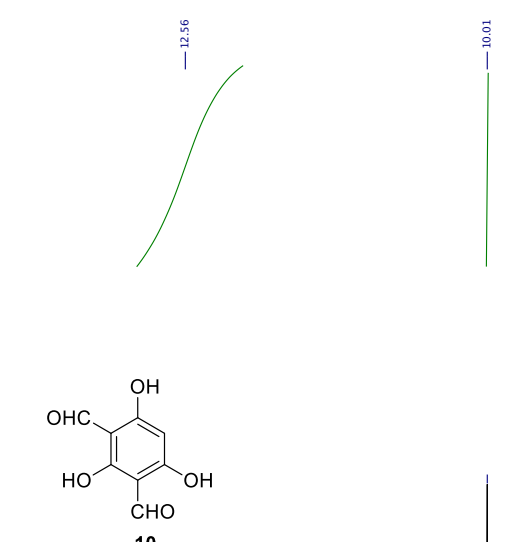

10

${ }^{1} \mathrm{H}$ NMR

$\mathrm{d}_{6}$-DMSO

$500 \mathrm{MHz}$
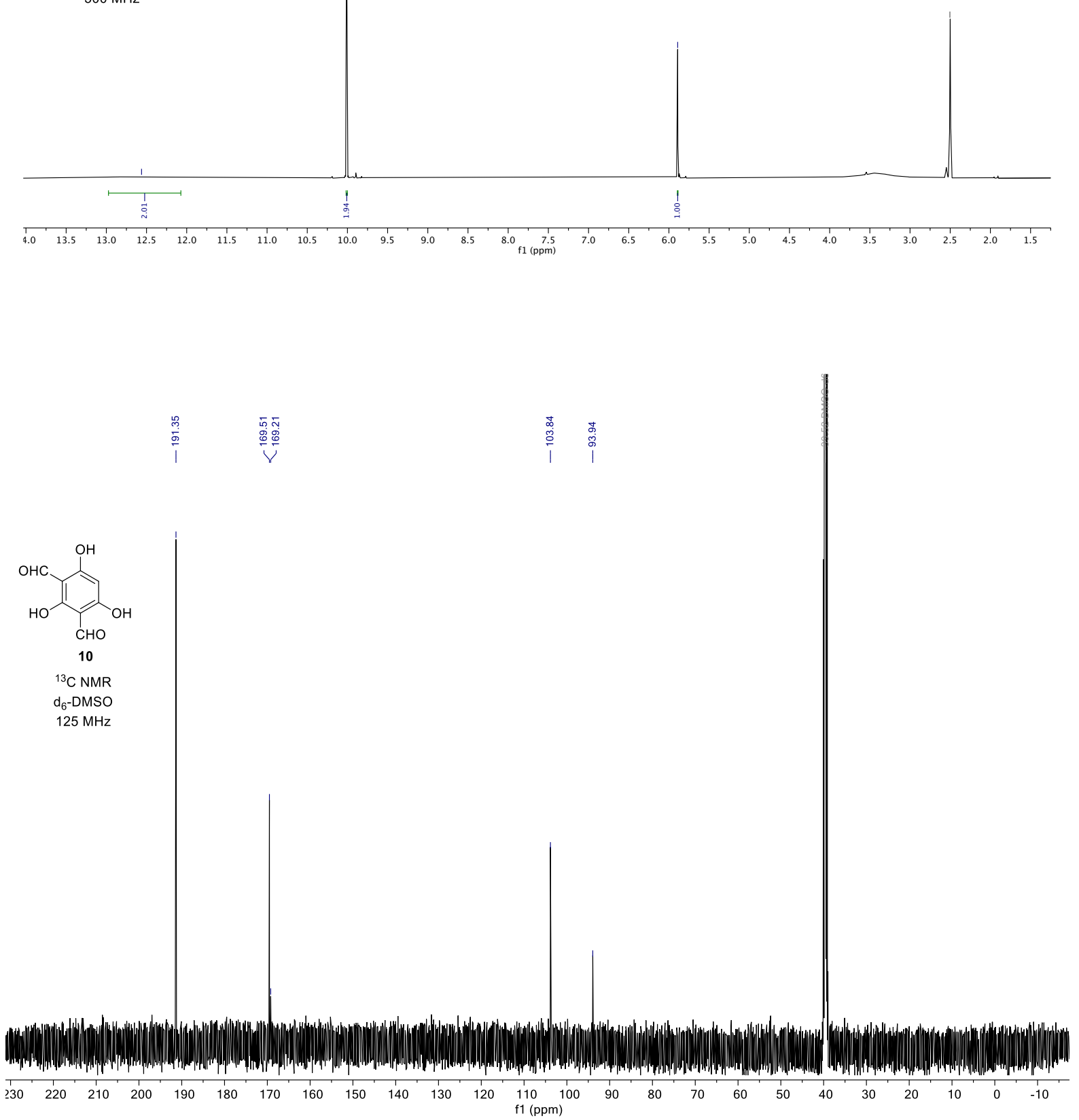

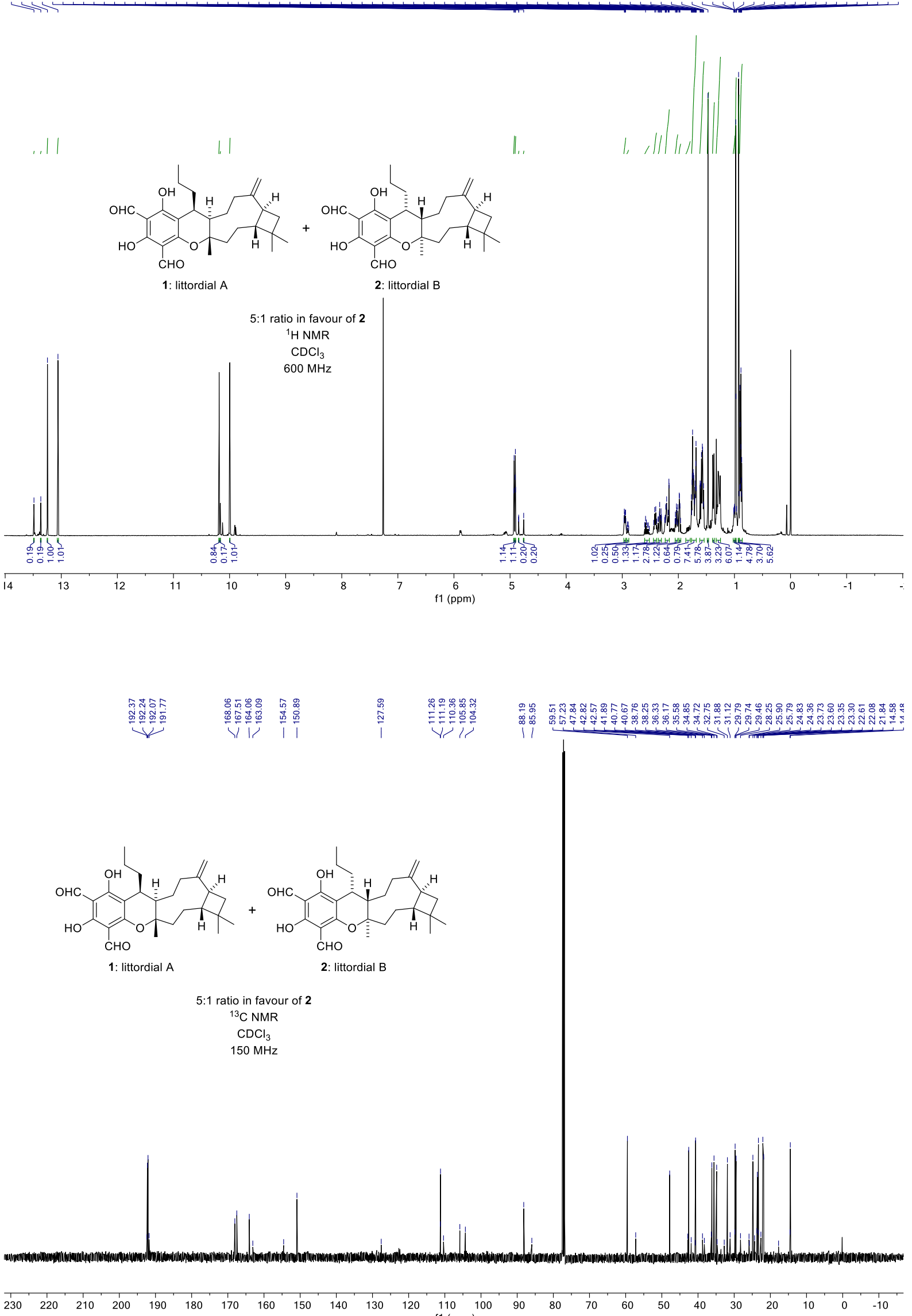


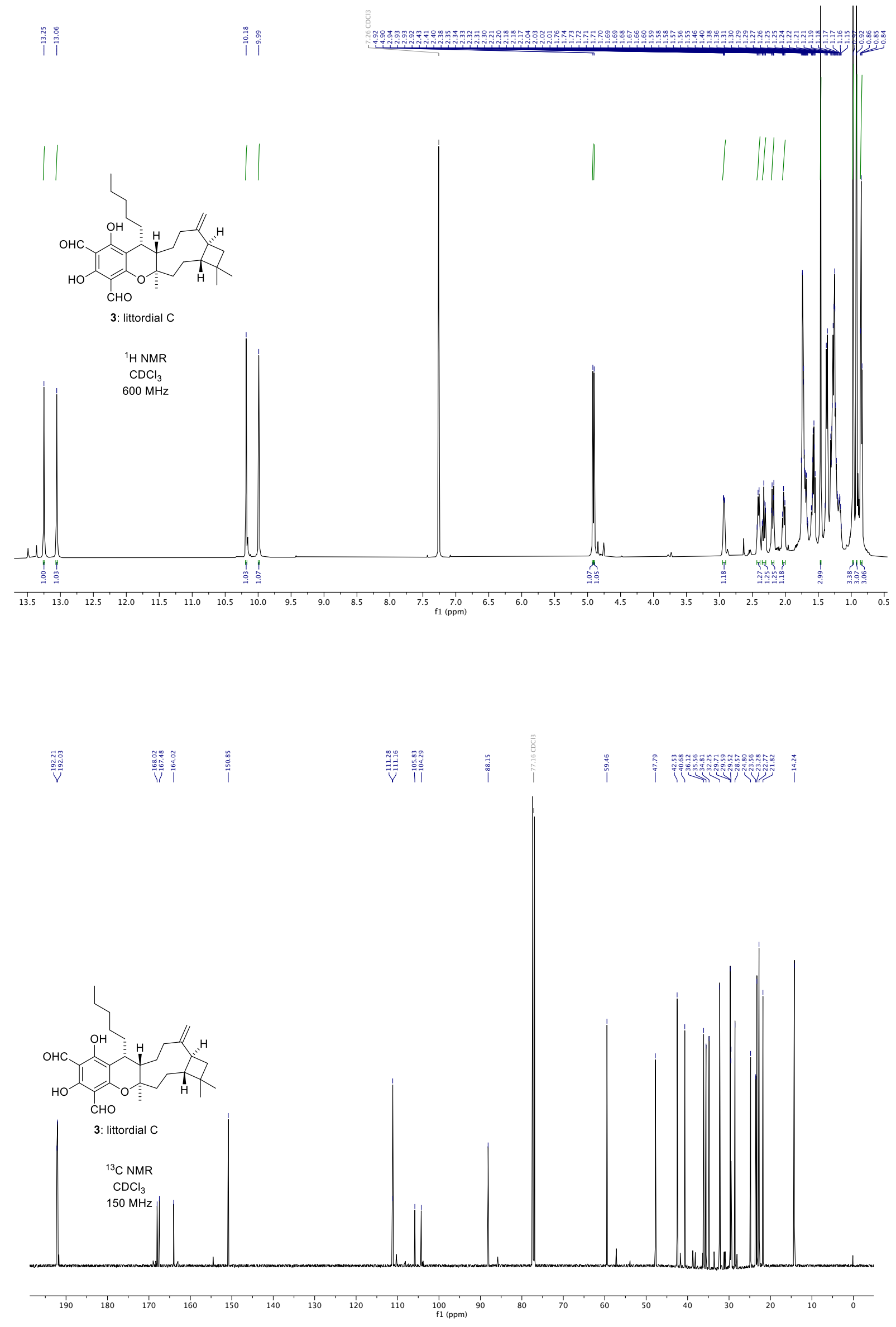



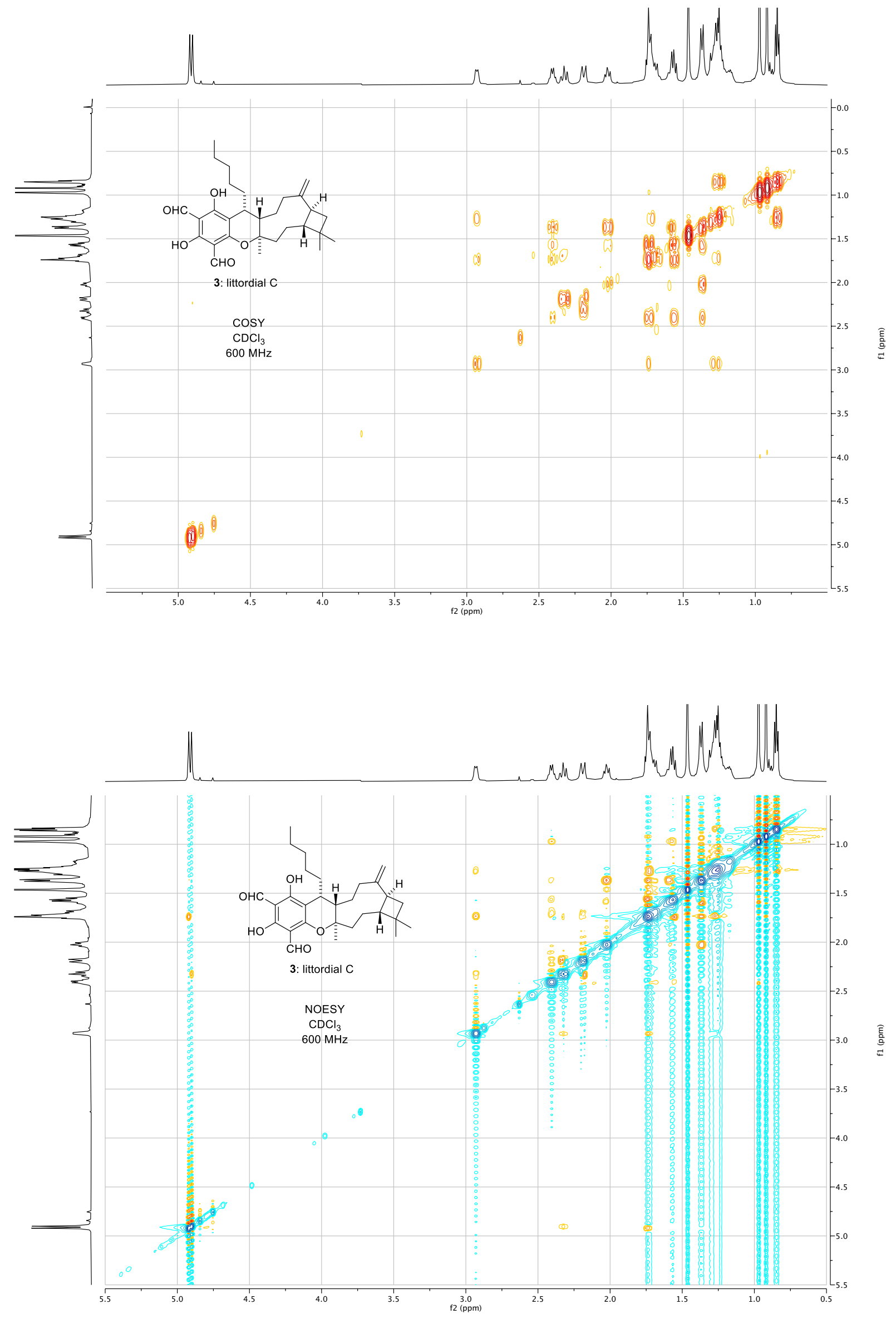

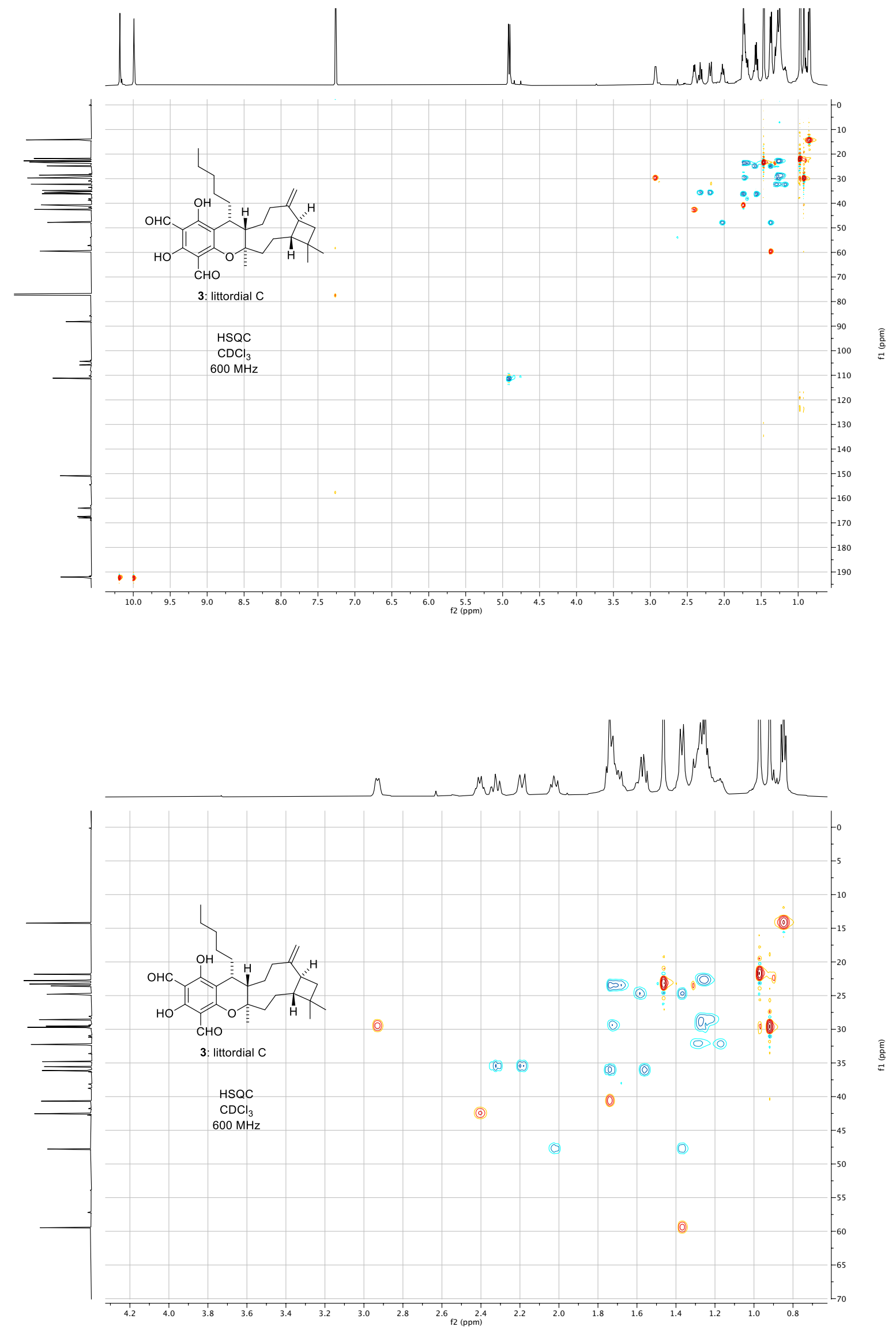

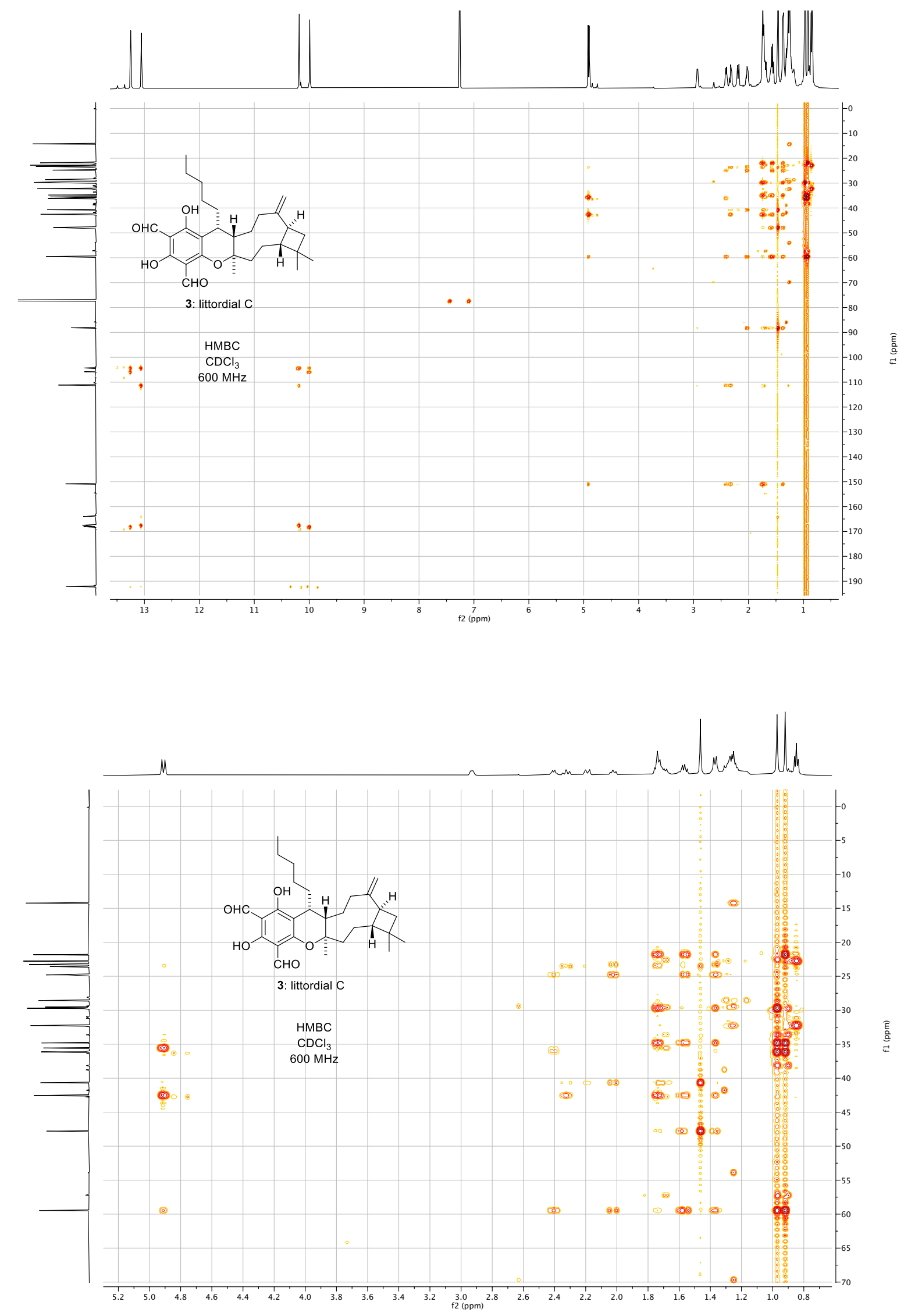

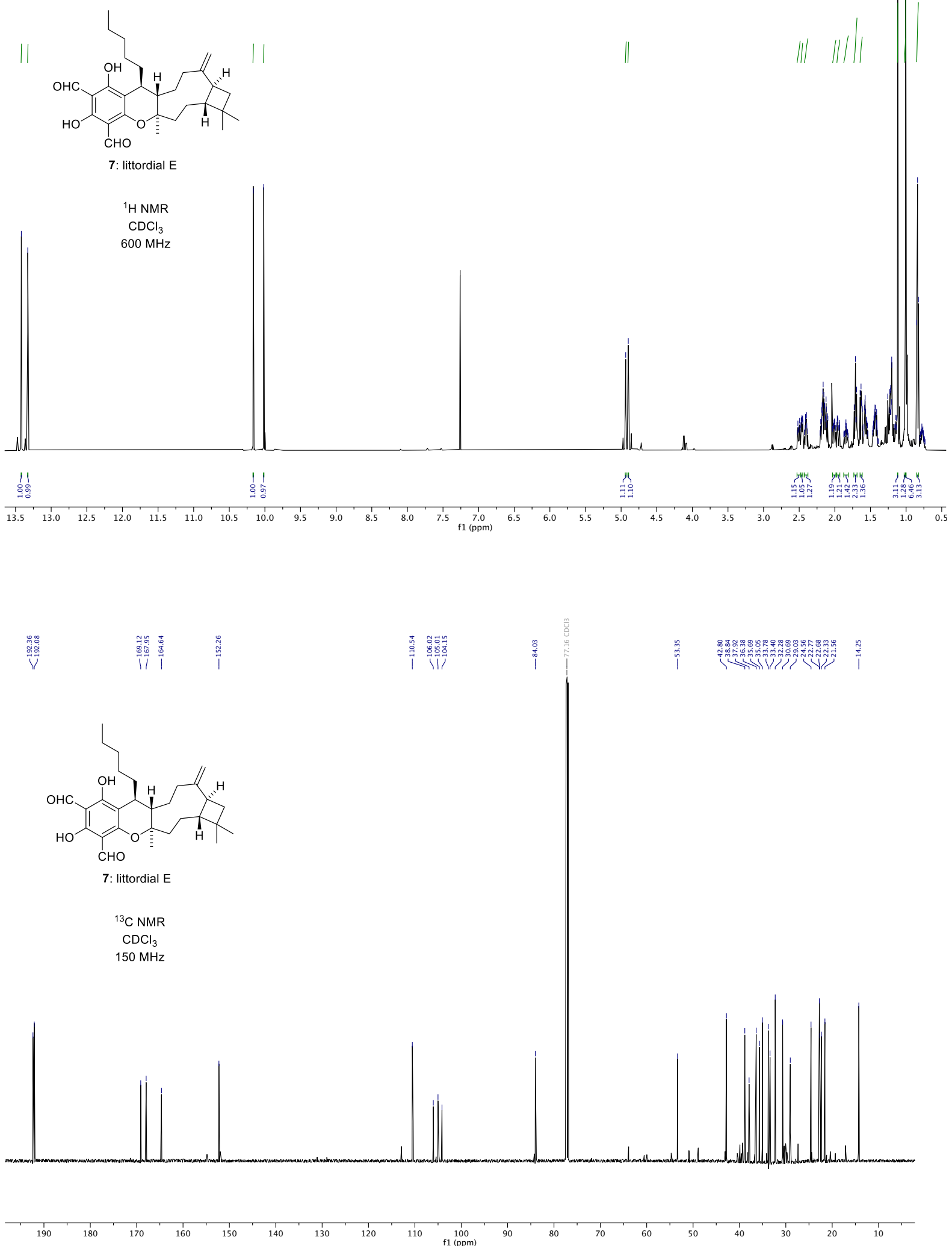

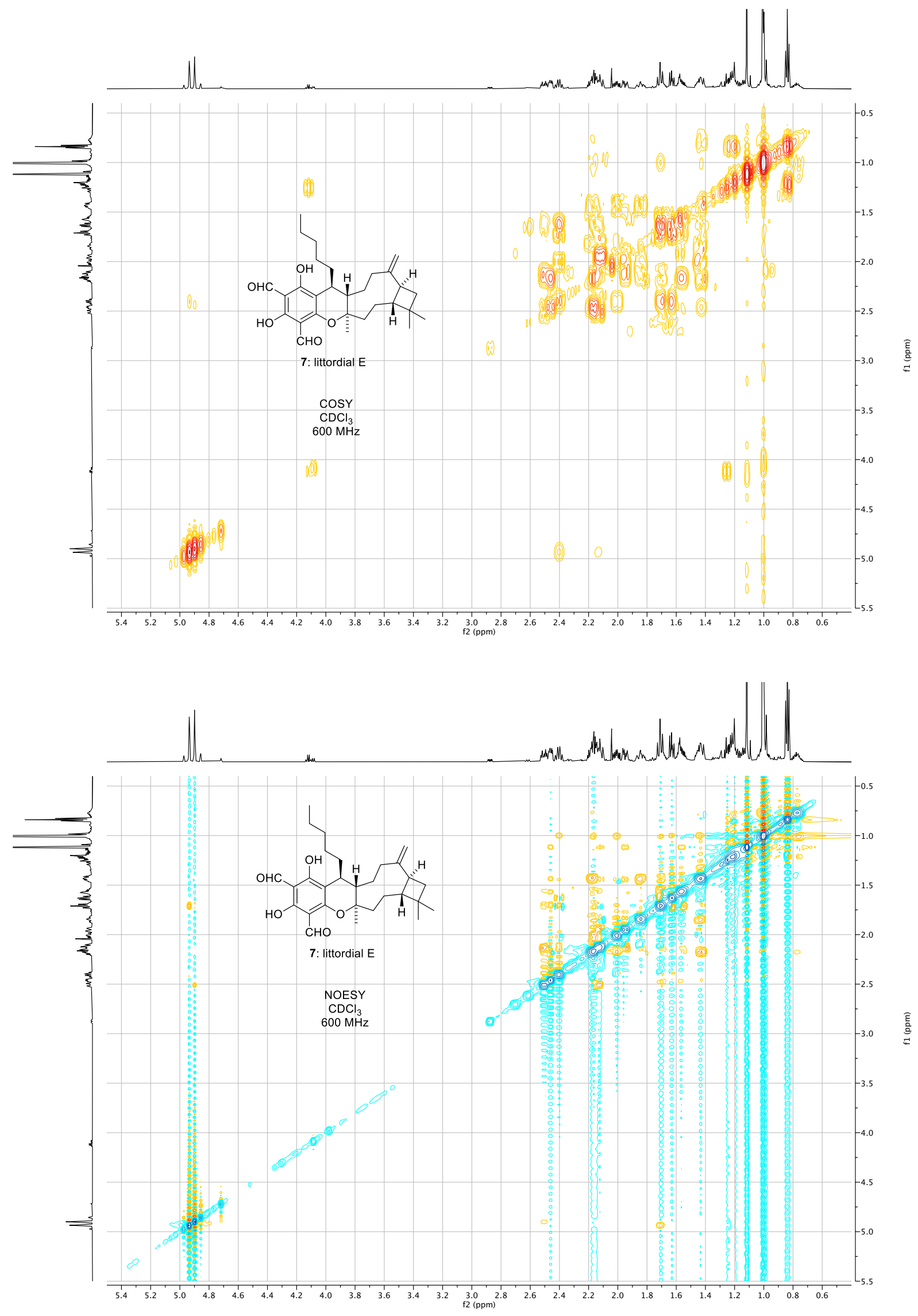

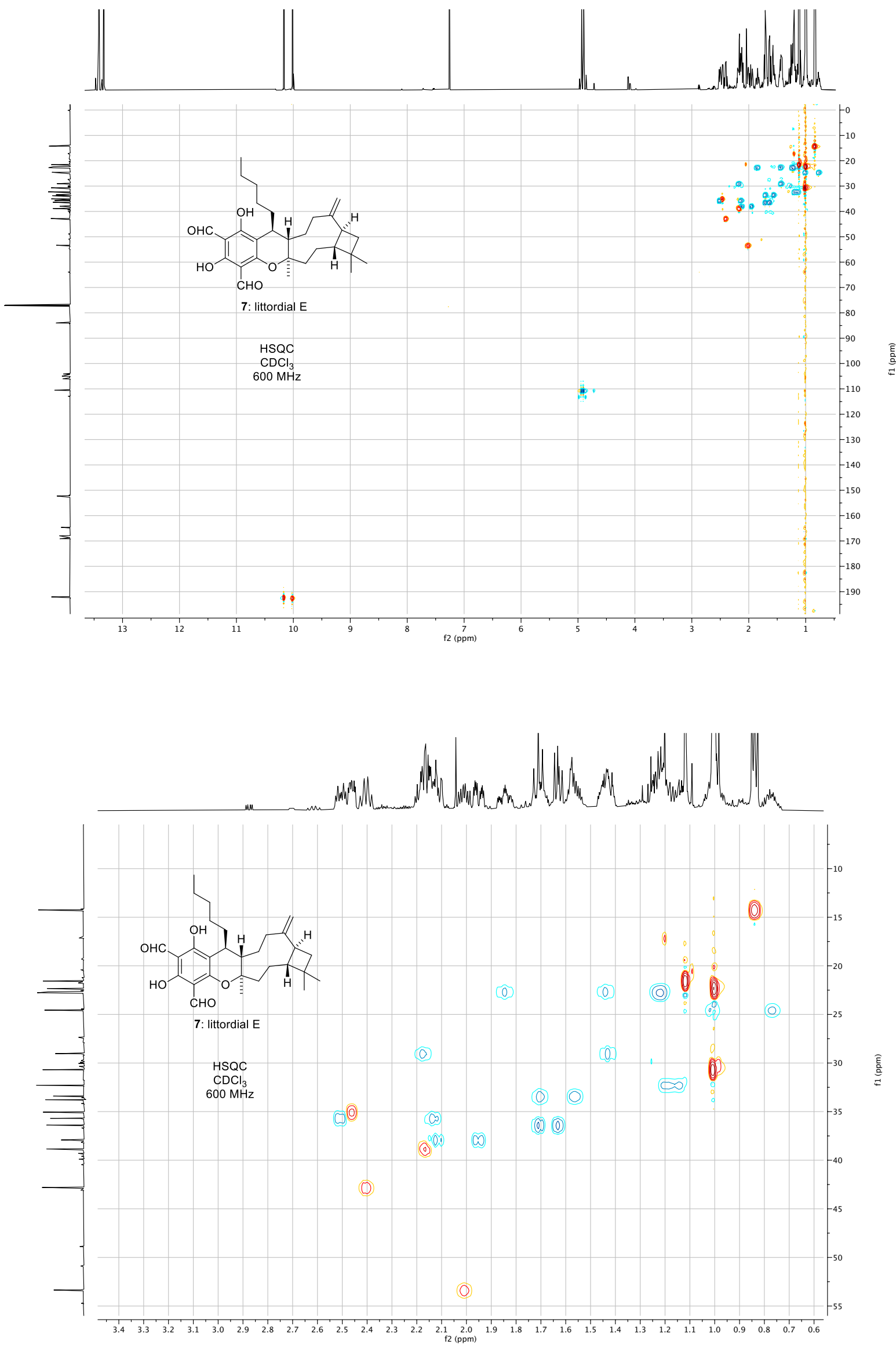

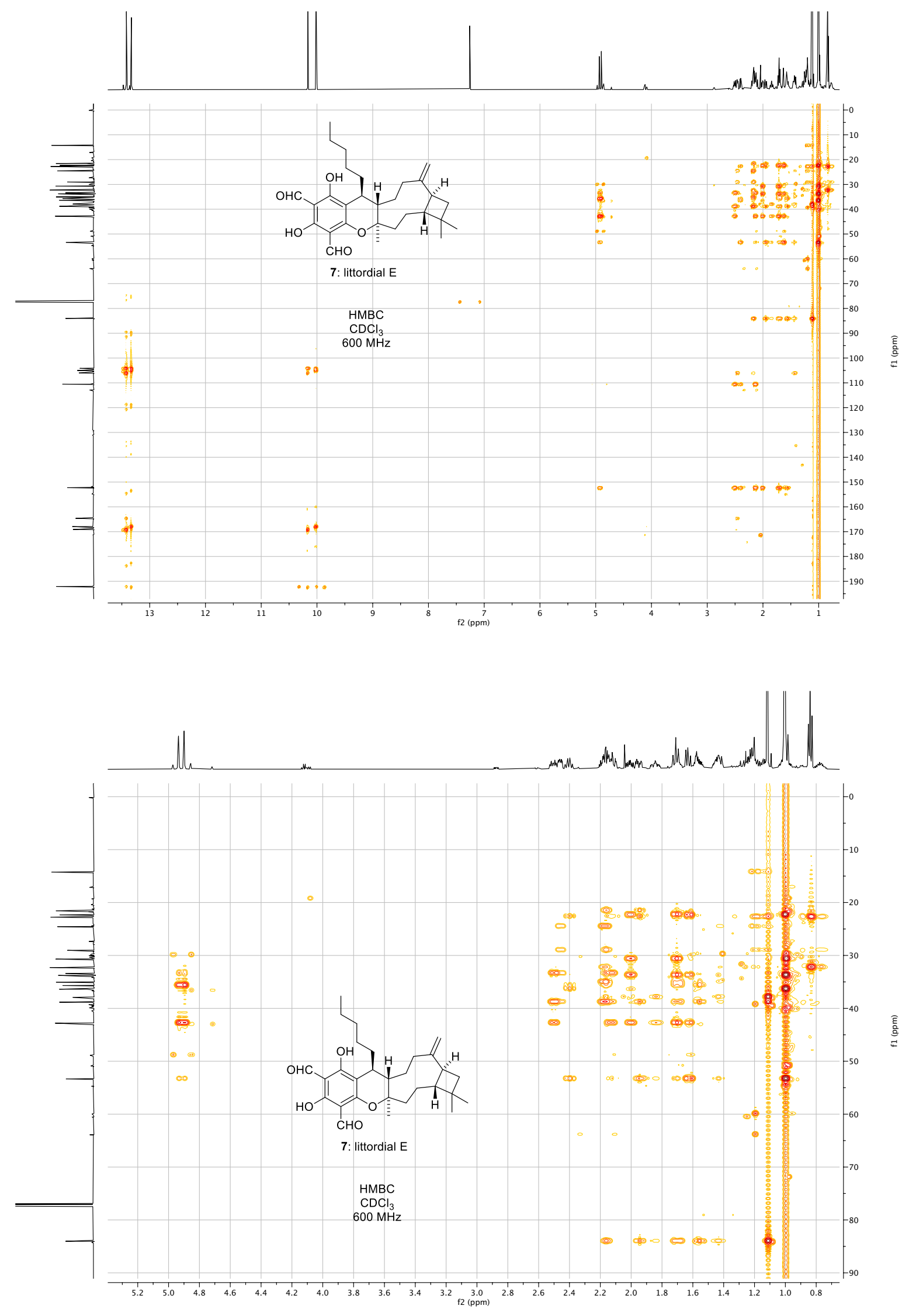

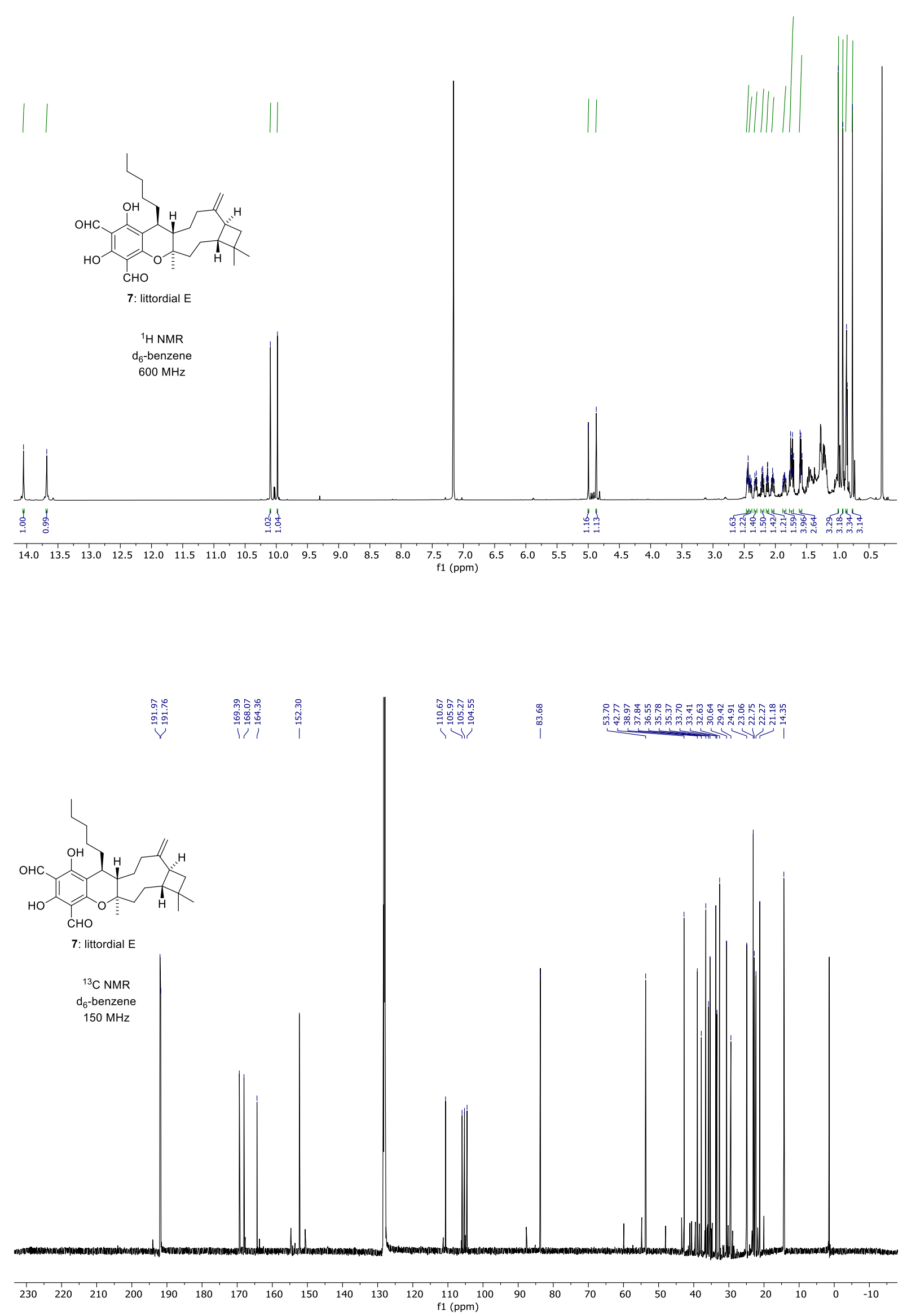

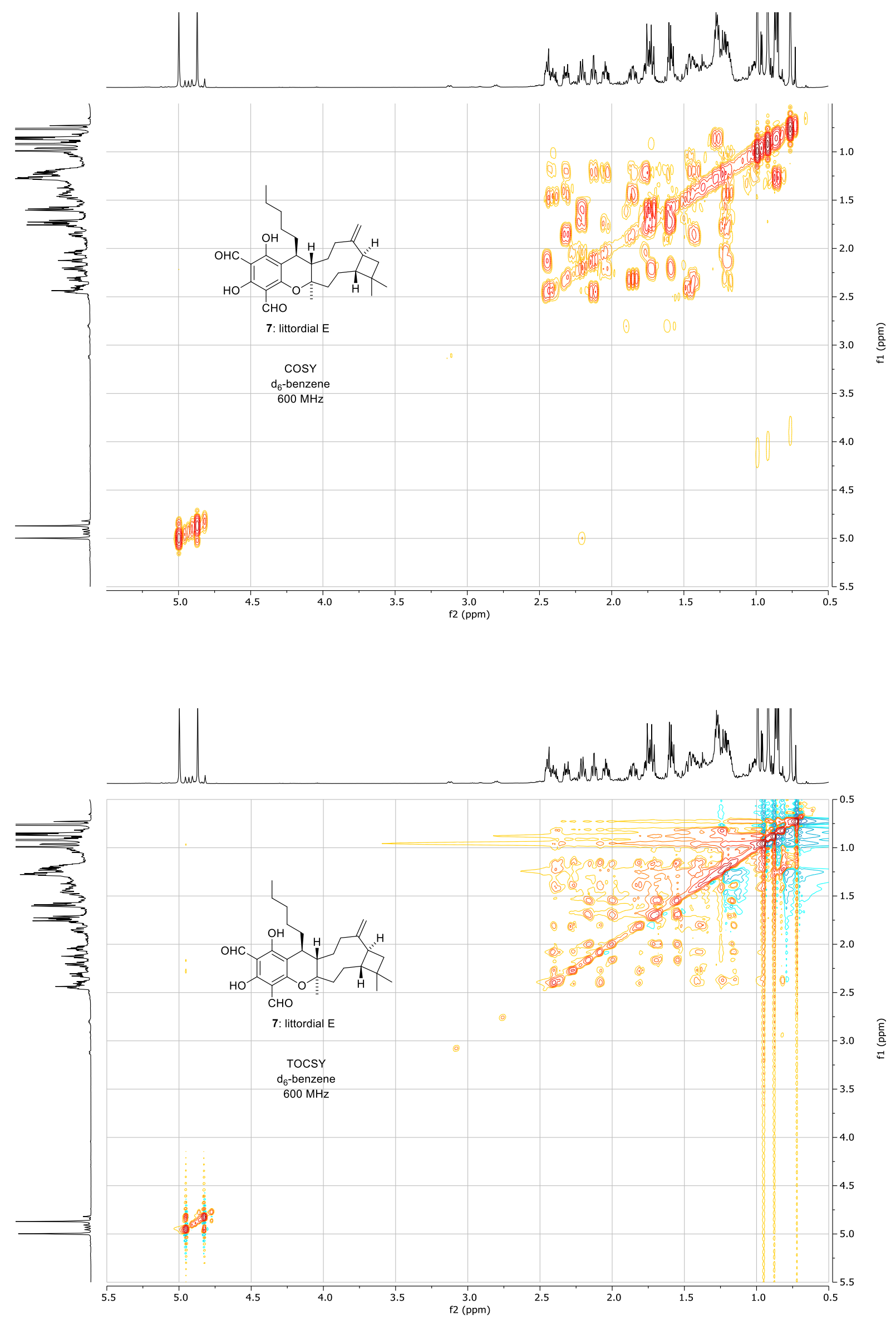

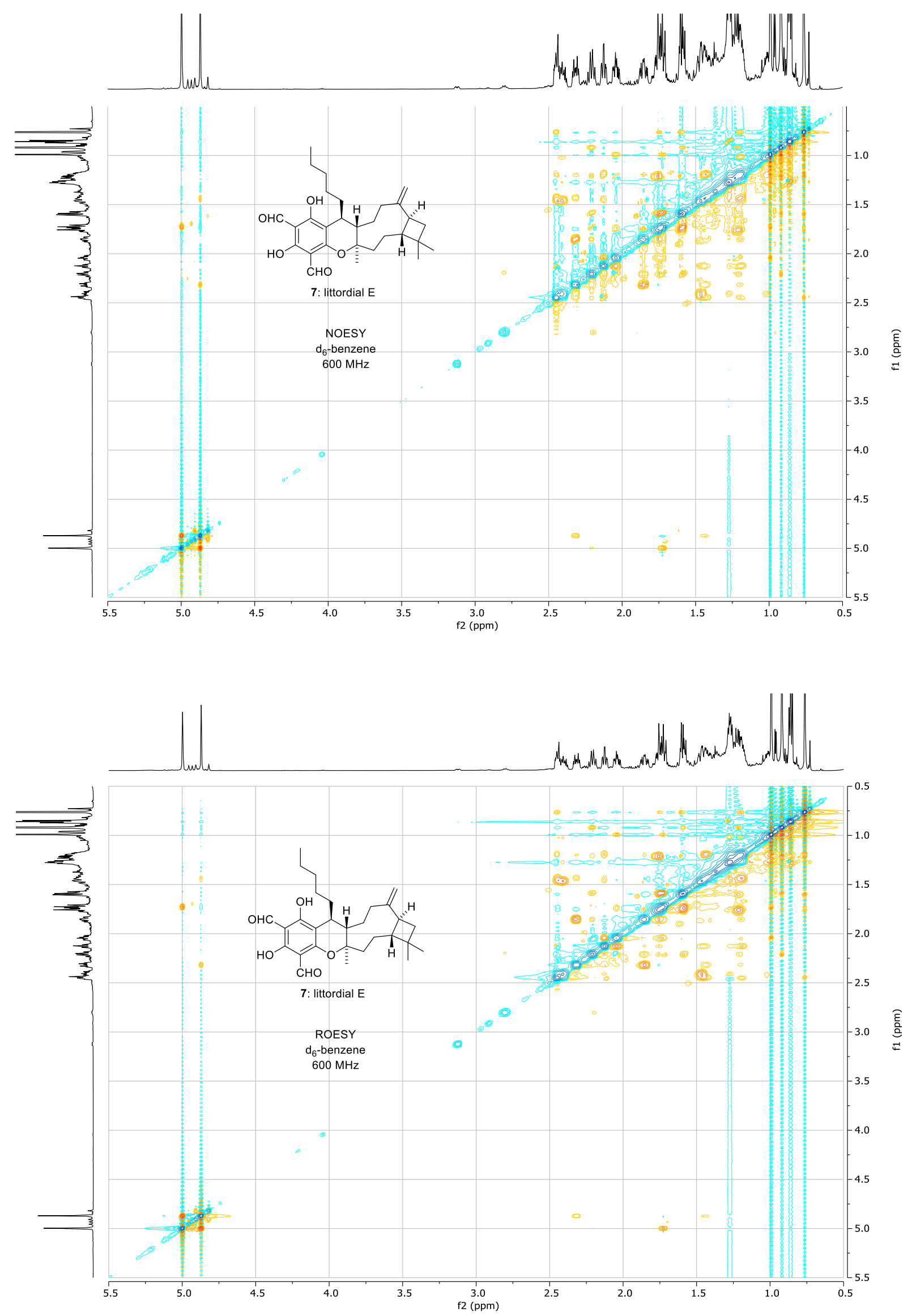


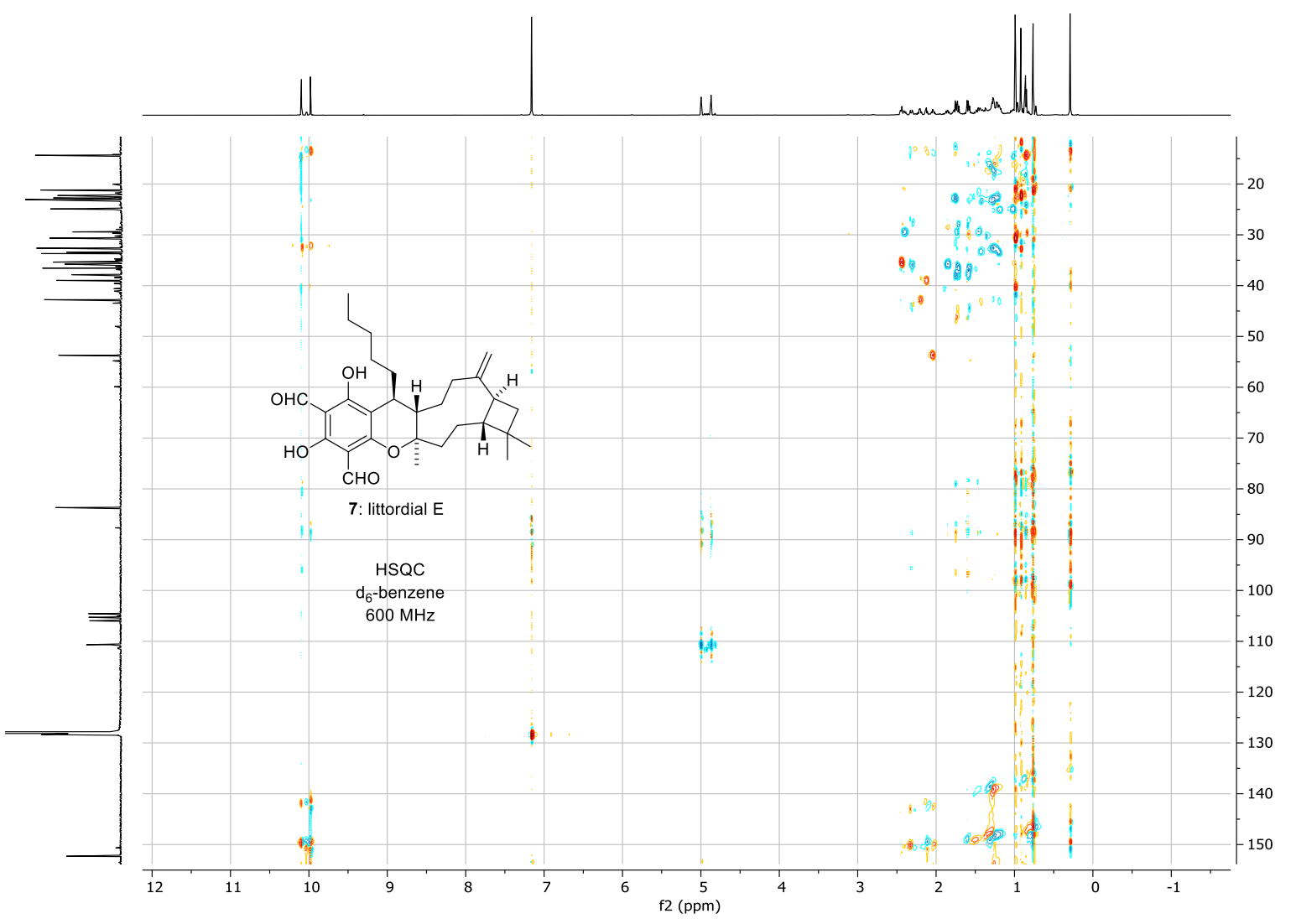

\section{$\underset{\mathrm{E}}{\stackrel{\mathrm{a}}{\mathrm{a}}}$}

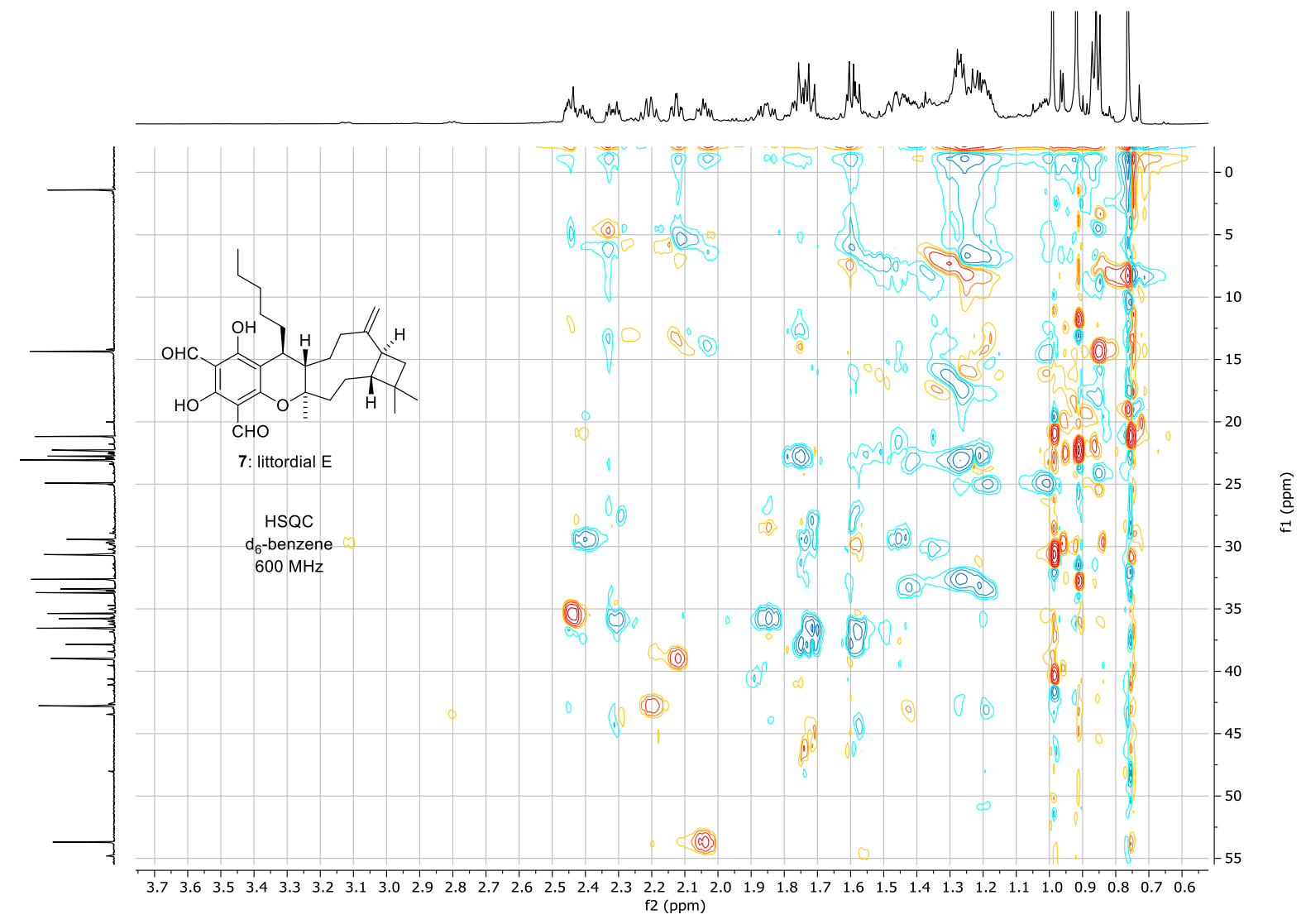




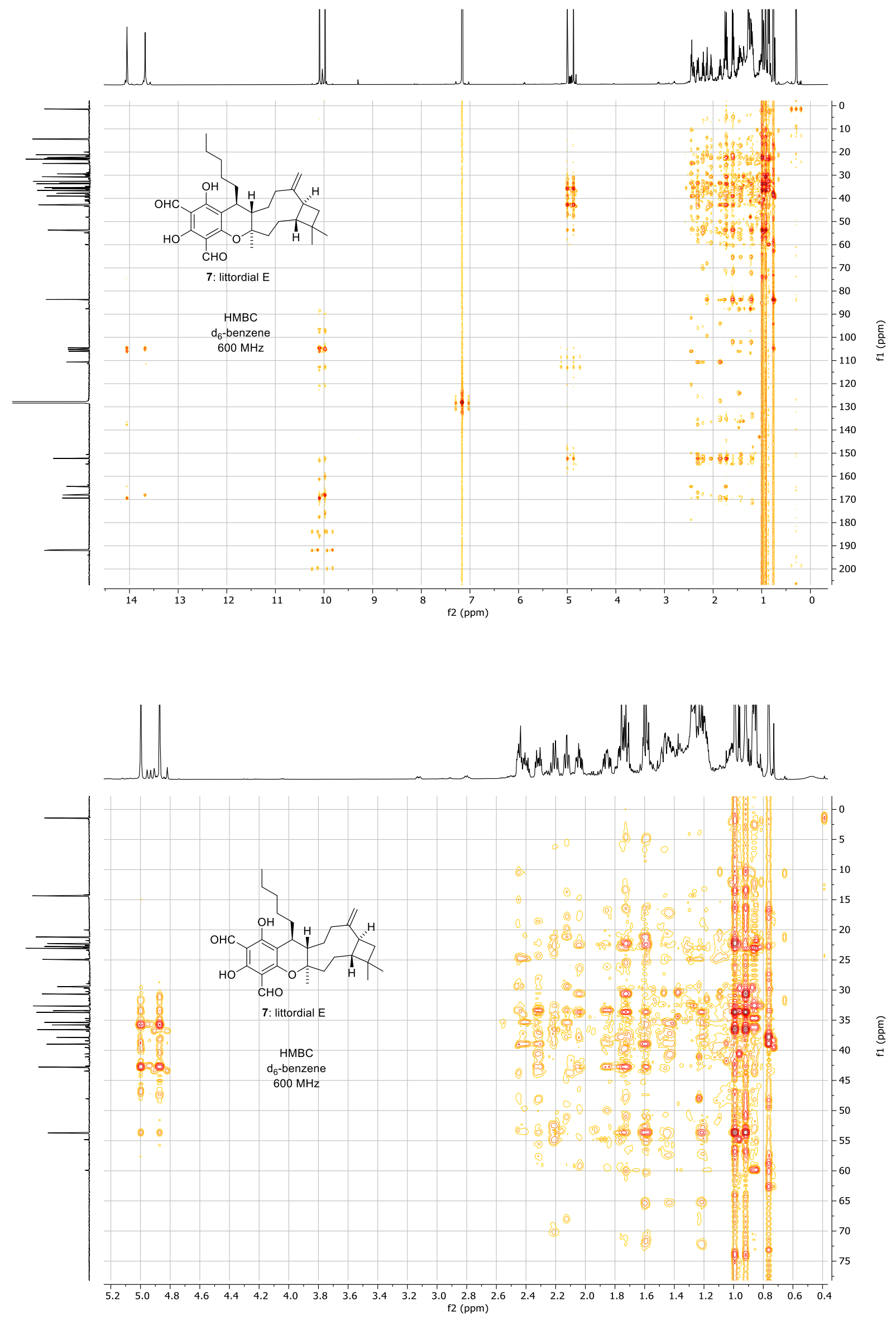


$\|$

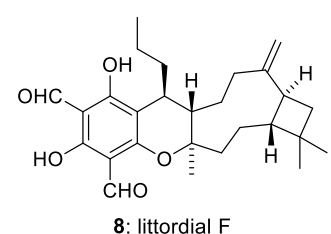

$\mathrm{CDCl}_{3}$

$600 \mathrm{MHz}$
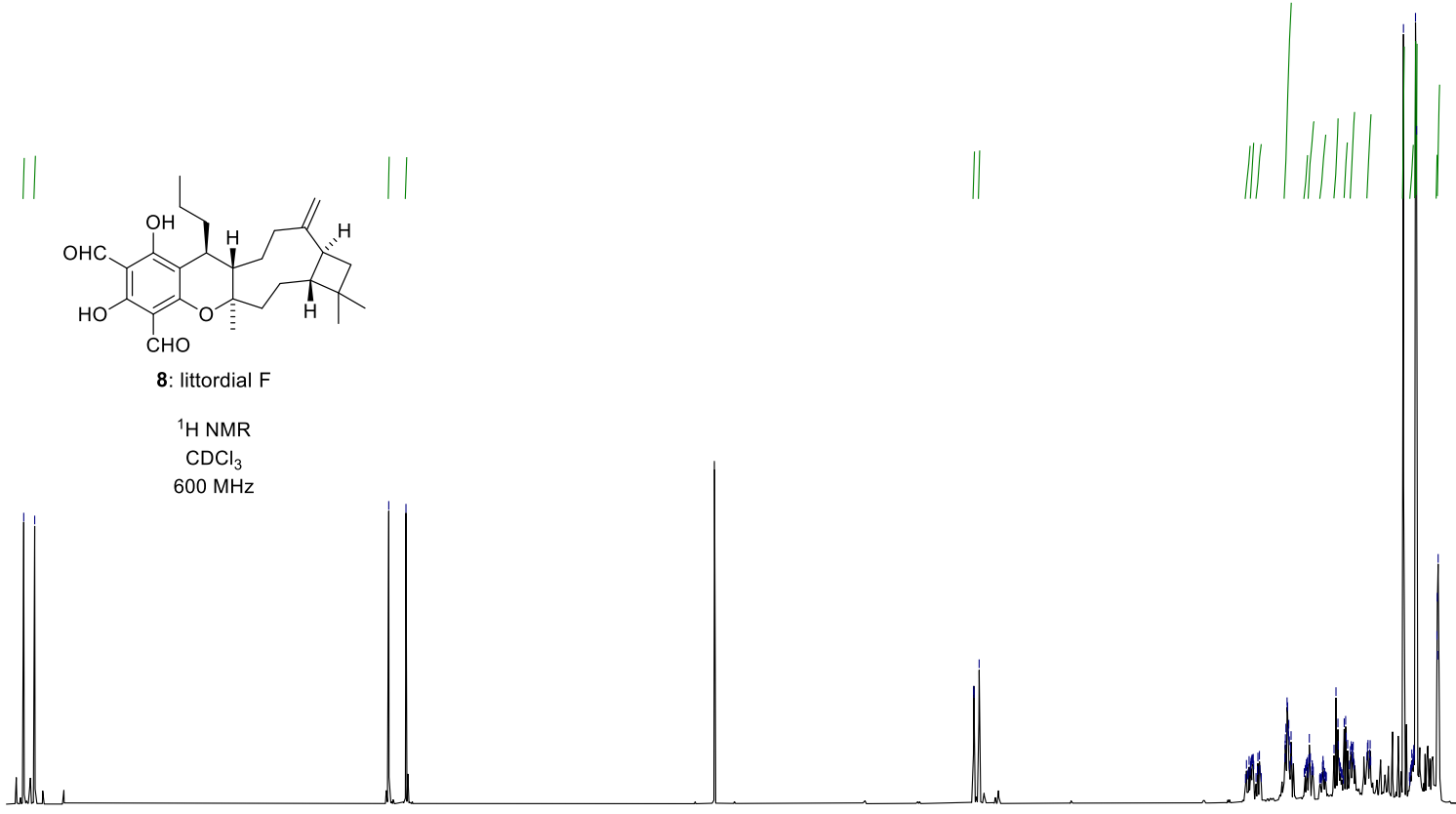

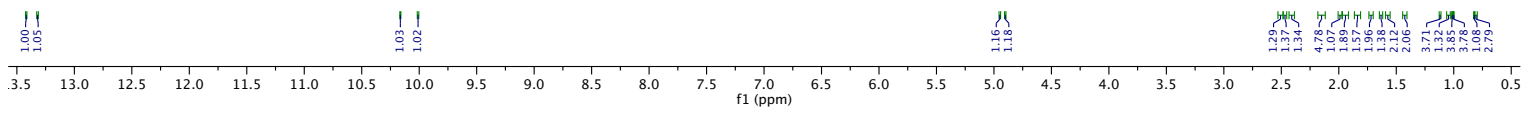
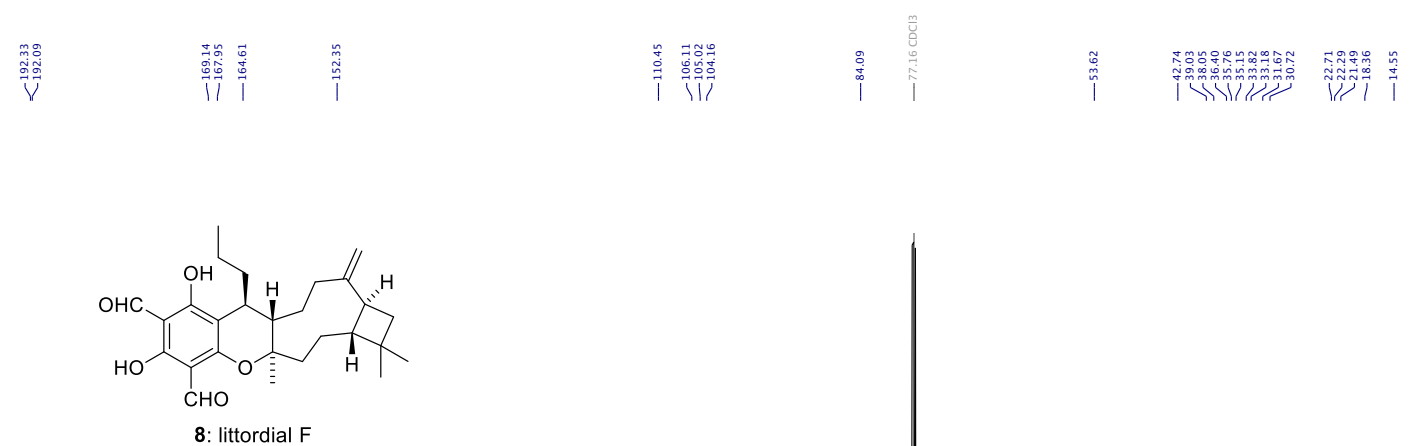

${ }^{13} \mathrm{C}$ NMR

$\mathrm{CDCl}_{3}$

$150 \mathrm{MHz}$
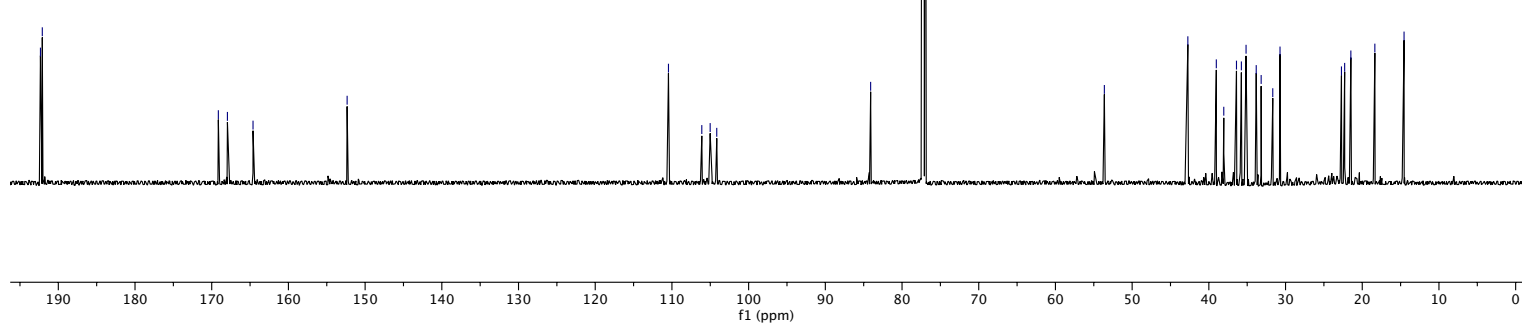

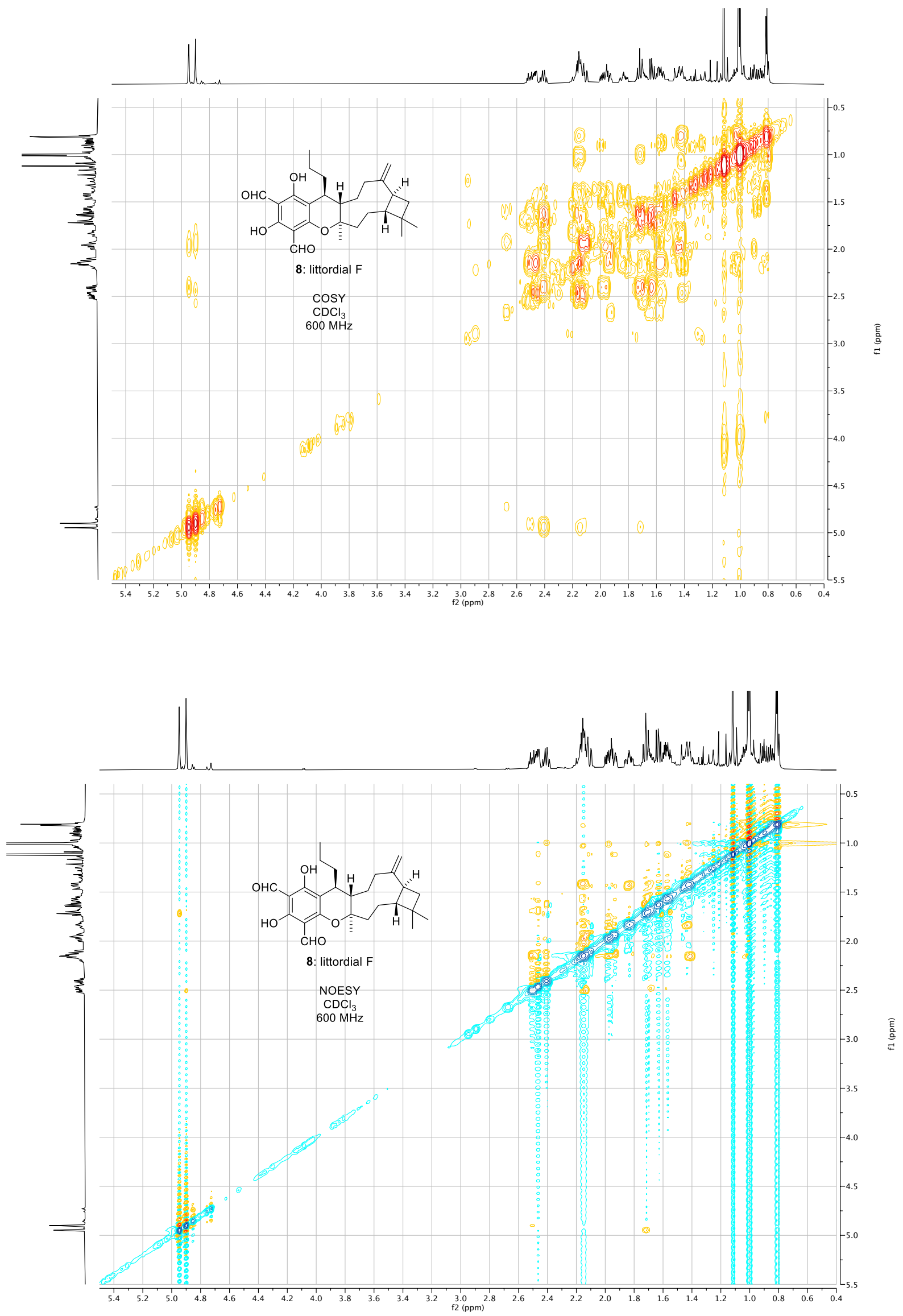

27 

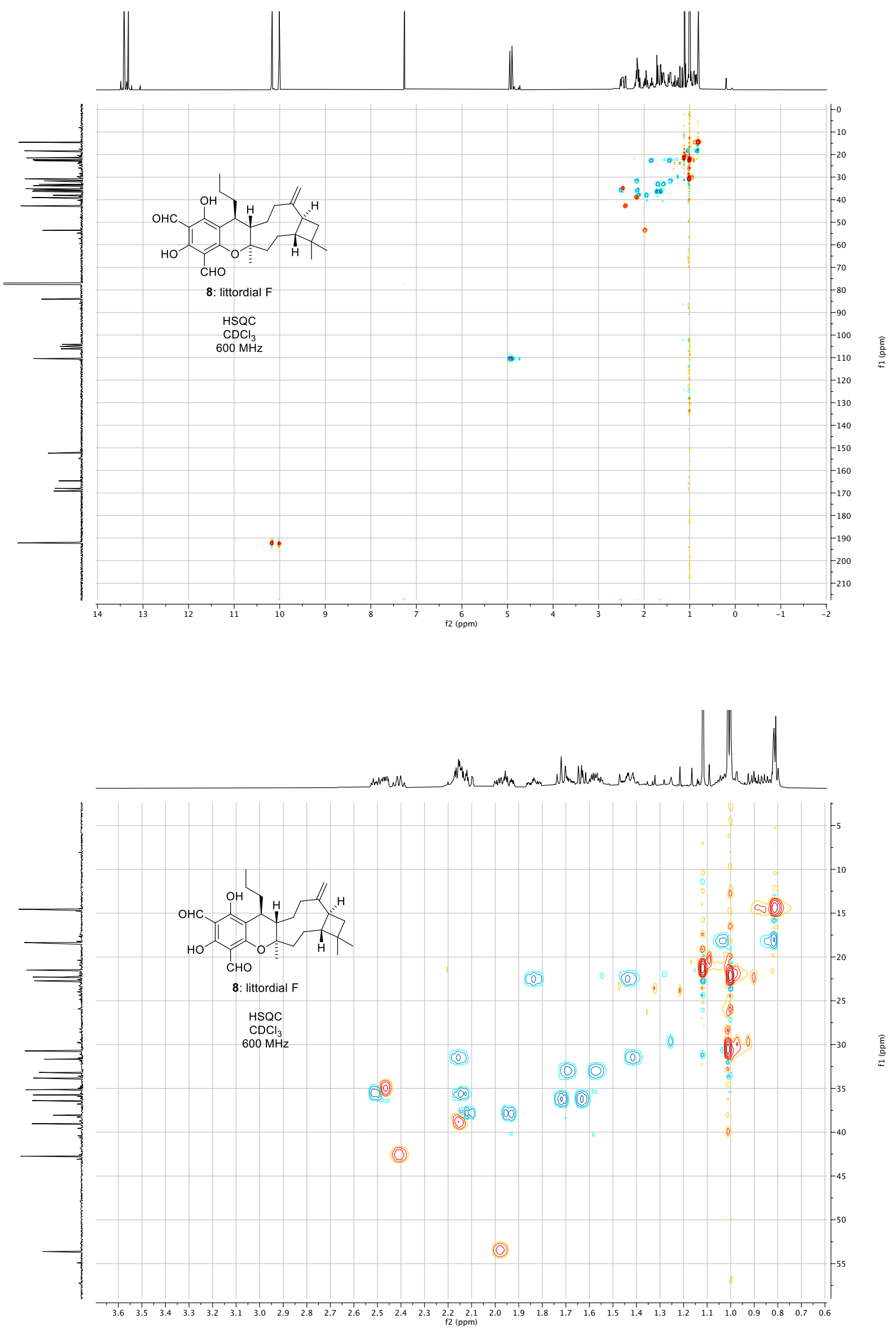

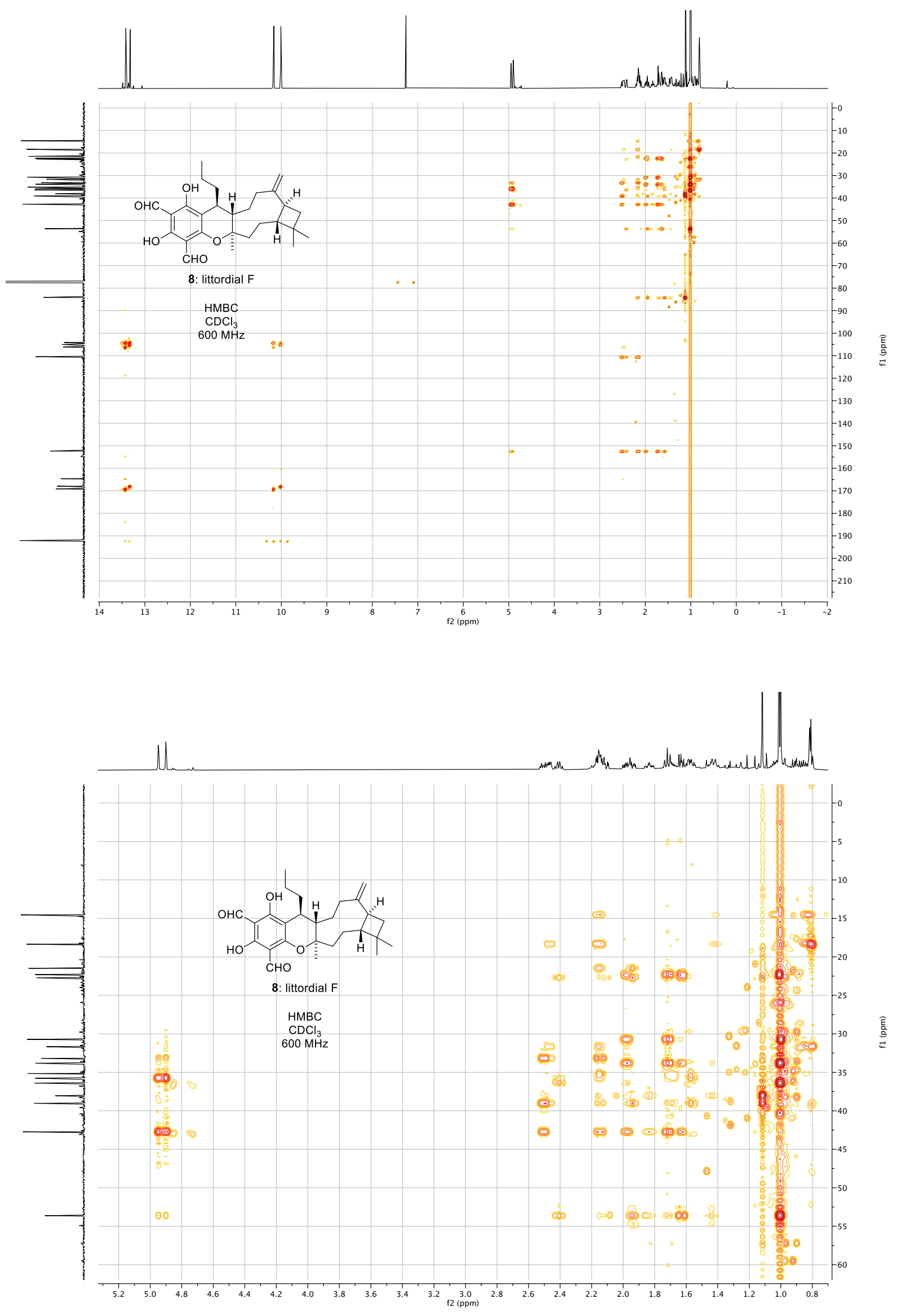

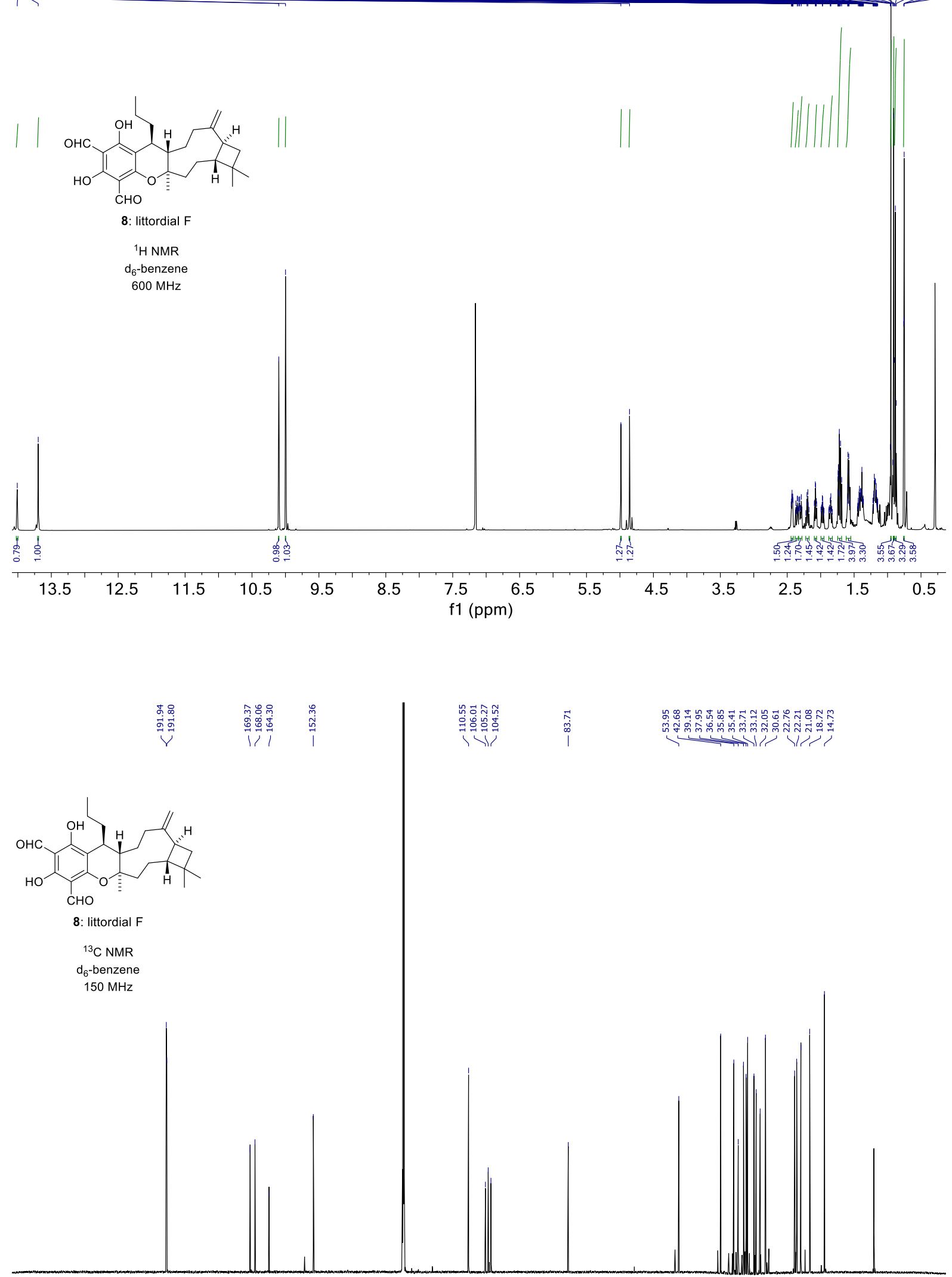

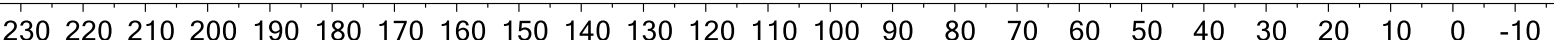
f1 (ppm) 

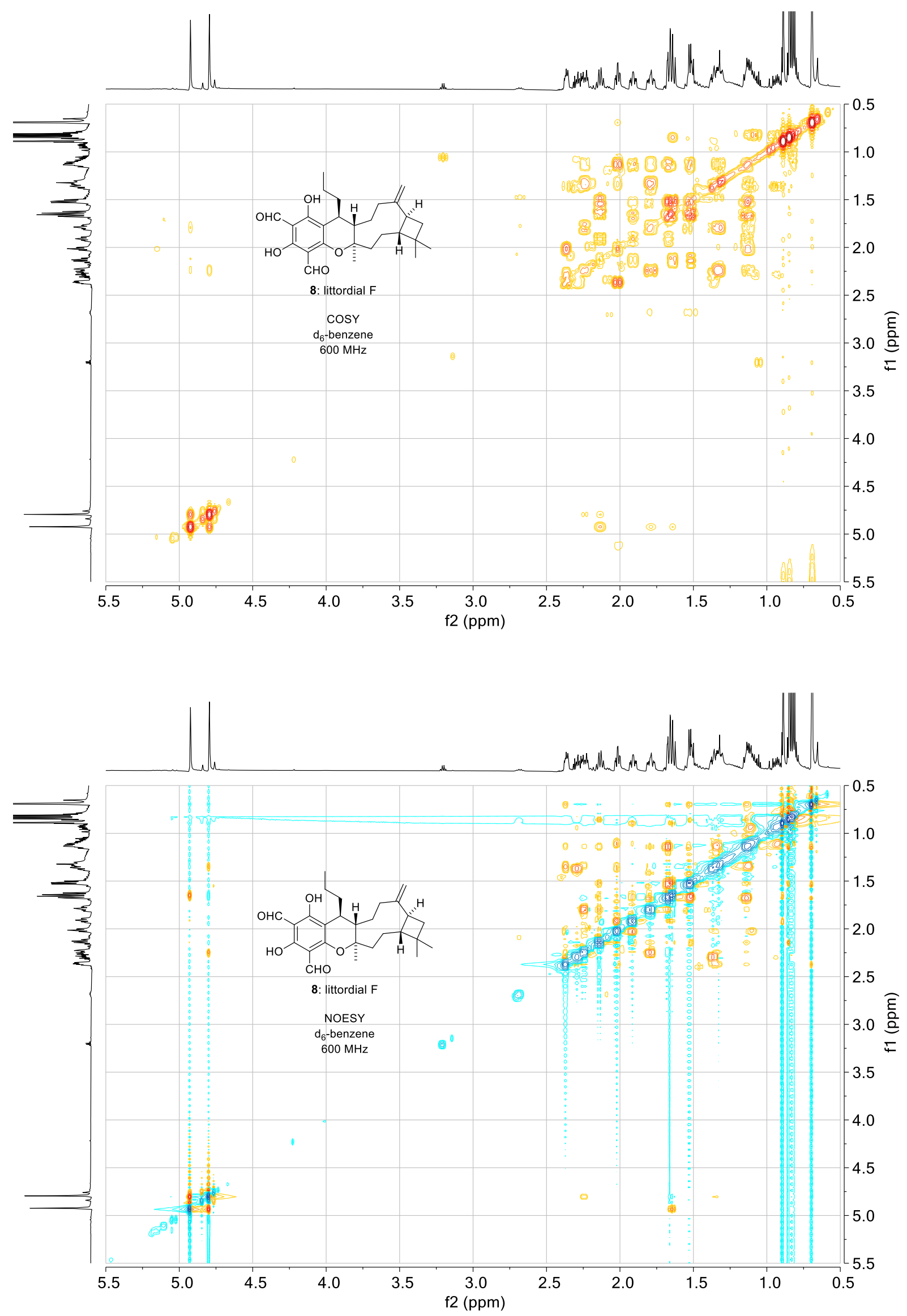

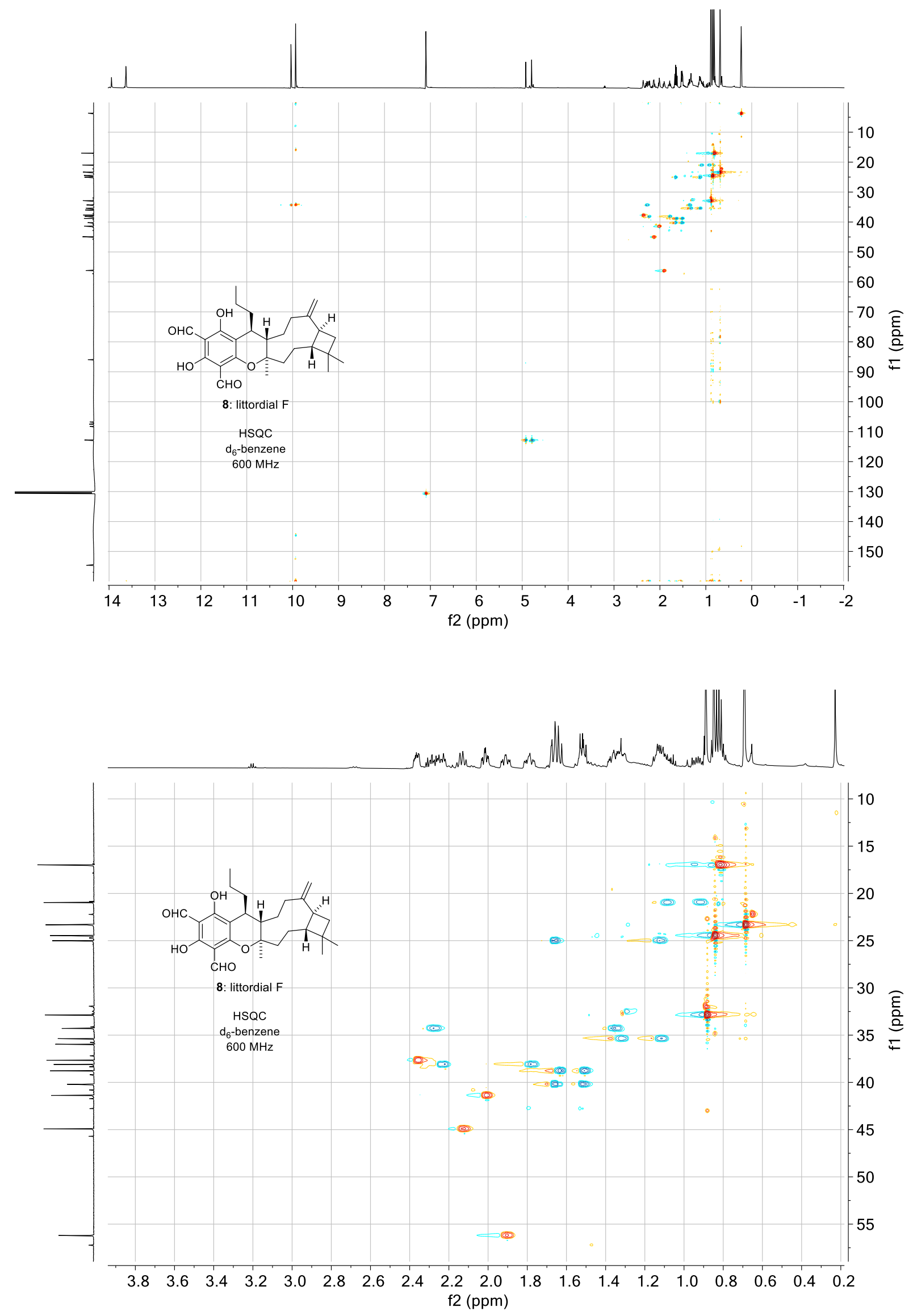

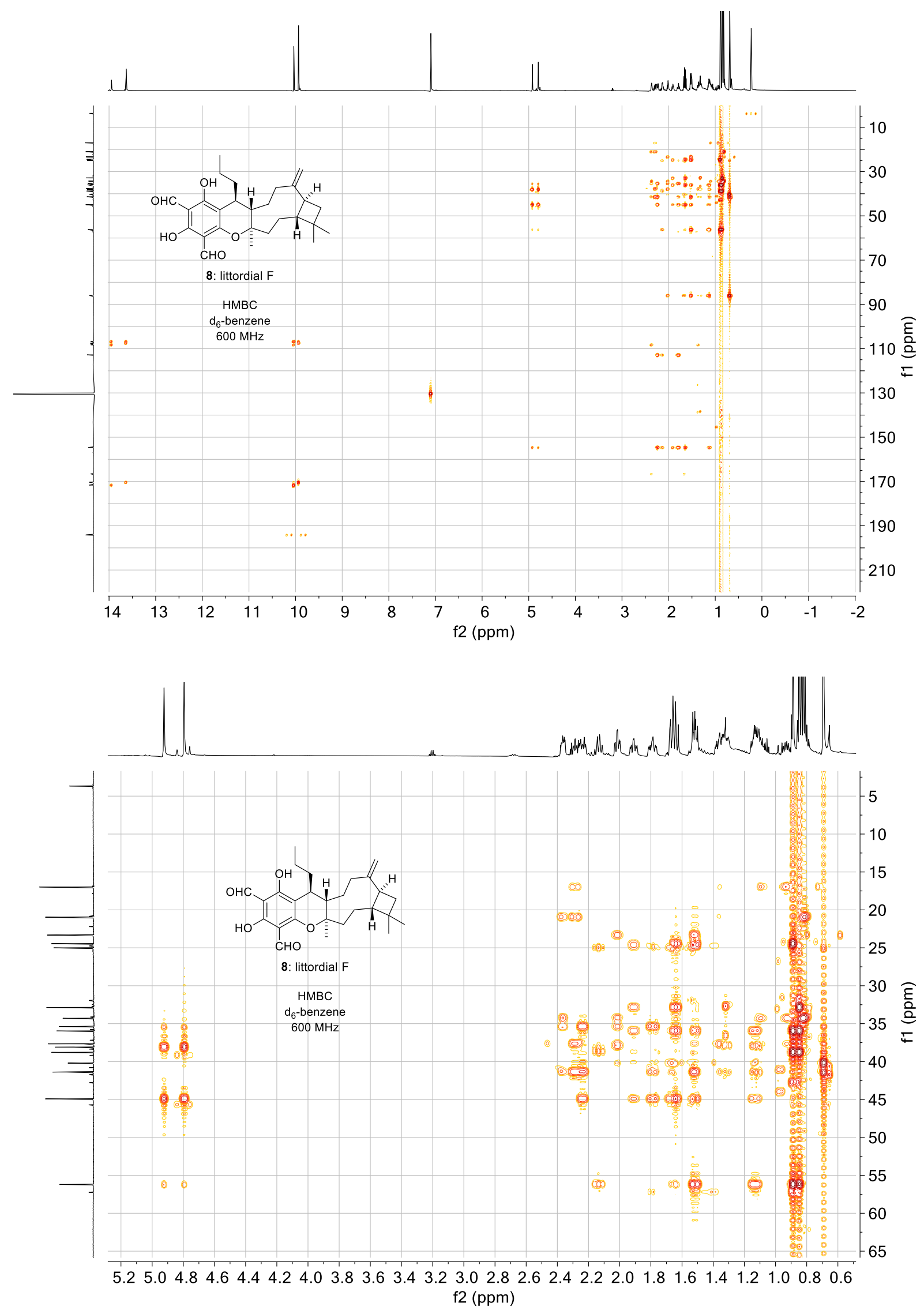


\section{Structural revision of littordial F}

The structural revision of littordial F (8) is described here and proceeds in a similar manner to what is written in the manuscript for littordial E (7). From the HSQC spectrum the coupling of C-9' to a single proton at $\delta=2.47$ is in contradiction to what is reported from the isolation, which assigns $\mathrm{C}-9^{\prime}$ as a $-\mathrm{CH}_{2}$ unit within their proposed 8 -membered ring. Furthermore, the authors also state $\mathrm{C}-10^{\prime}$ is a tertiary carbon but we see coupling to protons at $\delta=1.43$ and $\delta=$ 2.16. The COSY spectrum establishes correlations from $\mathrm{H}-9^{\prime} / \mathrm{H}-5 /-\mathrm{H}-6$ as previously seen in 7, connections which would be impossible in the isolation team's proposed structure. Their observed correlation between $\mathrm{H}-12^{\prime}$ to $\mathrm{H}-10^{\prime}$ is also questionable. Although a correlation to $\mathrm{H}-$ $10^{\prime}$ is seen, the significant overlap around $\delta=0.85-0.80 \mathrm{ppm}$ makes distinguishing between signals from $\mathrm{H}-12^{\prime}$ and $\mathrm{H}-11^{\prime}$ difficult. However, we do see a coupling from a multiplet around $\delta=1.03$ to $\mathrm{H}-10^{\prime}$, corresponding to the second proton of $\mathrm{C}-11^{\prime}$. Other signals around this region are designated as the gem-dimethyl located on the cyclobutene ring of caryophyllene making this assignment much less ambiguous.

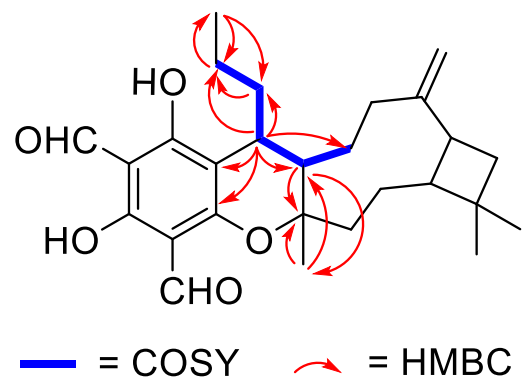

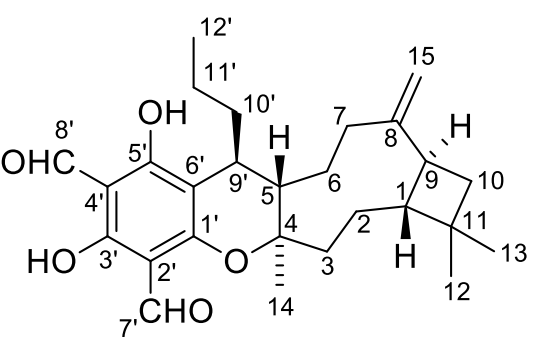

8: littordial $\mathrm{F}$ revised structure

Key 2D NMR correlations in the structure revision of littordial F

From the HMBC spectrum, we see many of the same correlations present in the spectrum for 7 including $\mathrm{H}-9^{\prime}$ to $\mathrm{C}-1^{\prime} / \mathrm{C}-6^{\prime} / \mathrm{C}-5 / \mathrm{C}-6, \mathrm{H}-5$ to C-4/C-14 and $\mathrm{H}-14$ to C-4/C-5, which were used to establish the six-membered ring. The proximity of the proton signal of $\mathrm{H}-9^{\prime}(\delta=2.47)$ to that of H-7 $(\delta=2.51)$, which shows an exceptionally strong correlation to C-5/C-6, makes detecting the coupling of $\mathrm{H}-9^{\prime}$ to $\mathrm{C}-5 / \mathrm{C}-6$ difficult, contributing to the authors' misassigned structure. The correlation from $\mathrm{H}-9^{\prime}$ to $\mathrm{C}-6$ is particularly important, as in the isolated compound this would represent a five-bond correlation, so the fact that it is seen in our data is further evidence of the presence of a six-membered ring in $\mathbf{8}$. Finally, the $n$-propyl side chain of 8 is established by the correlations $\mathrm{H}-9^{\prime}$ to $\mathrm{C}-10^{\prime} / \mathrm{C}-11^{\prime}, \mathrm{H}-10^{\prime}$ to $\mathrm{C}-11^{\prime}, \mathrm{H}-11^{\prime}$ to $\mathrm{C}-10^{\prime} / \mathrm{C}-12^{\prime}$ and $\mathrm{H}-12^{\prime}$ to $\mathrm{C}-10^{\prime} / \mathrm{C}-11^{\prime}$. We observe the same NOESY correlations for 8 that are also seen in 7. Spectra of $\mathbf{8}$ obtained in $\mathrm{d}_{6}$-benzene is consistent with spectra collected in $\mathrm{CDCl}_{3}$ as well as data for compound $\mathbf{7}$. Therefore, $\mathbf{7}$ and $\mathbf{8}$ are assigned the same relative configurations. 


.

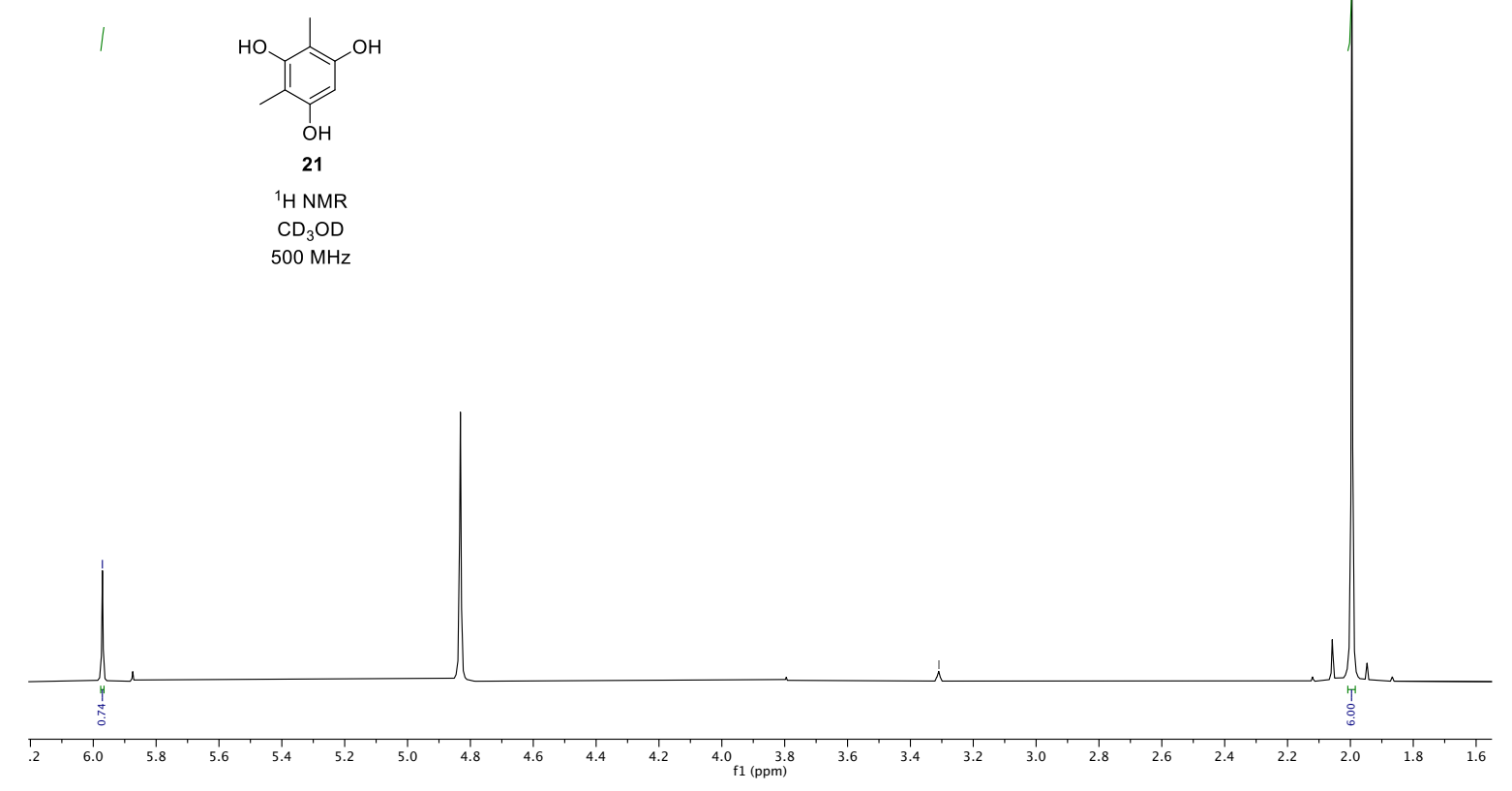

i

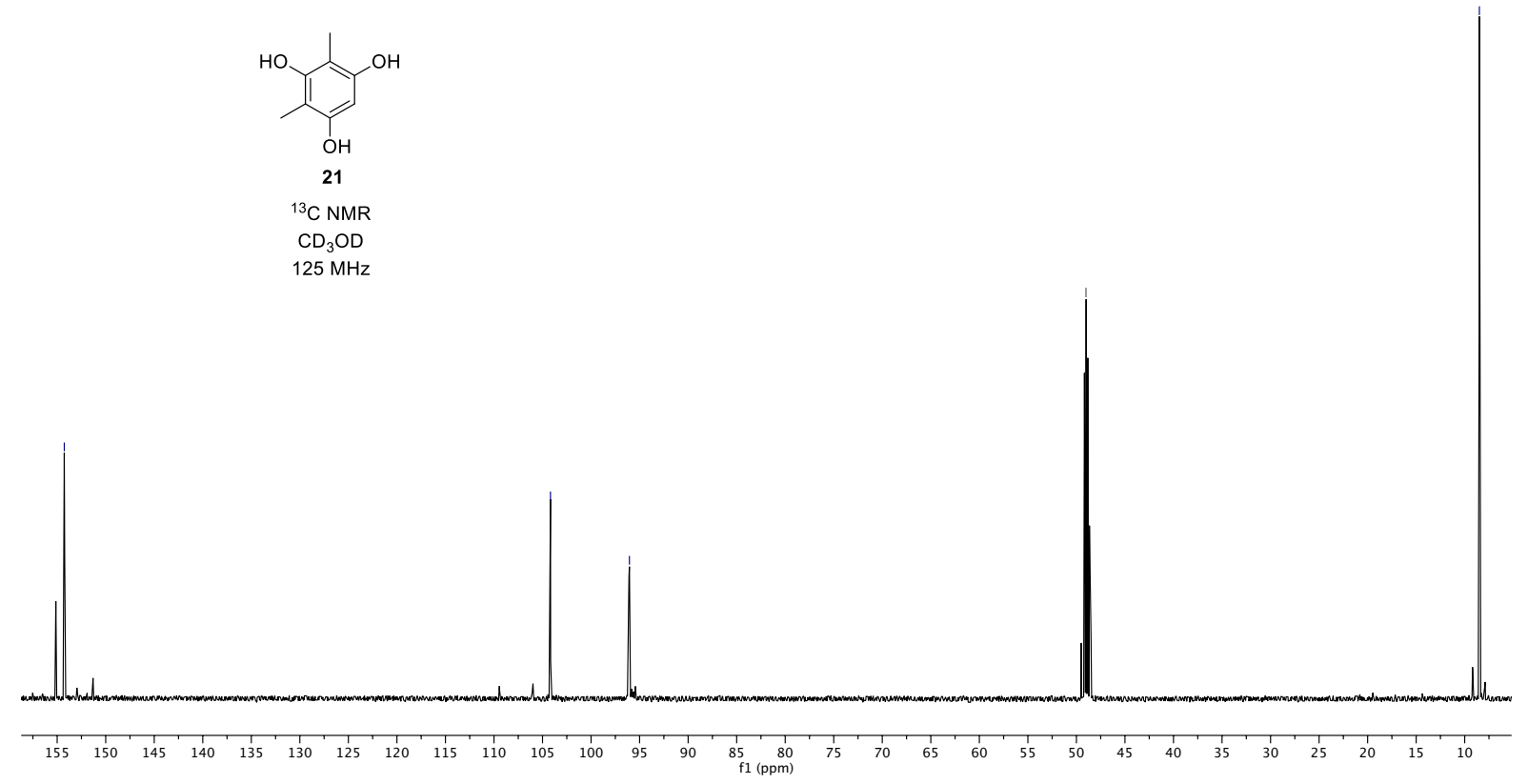



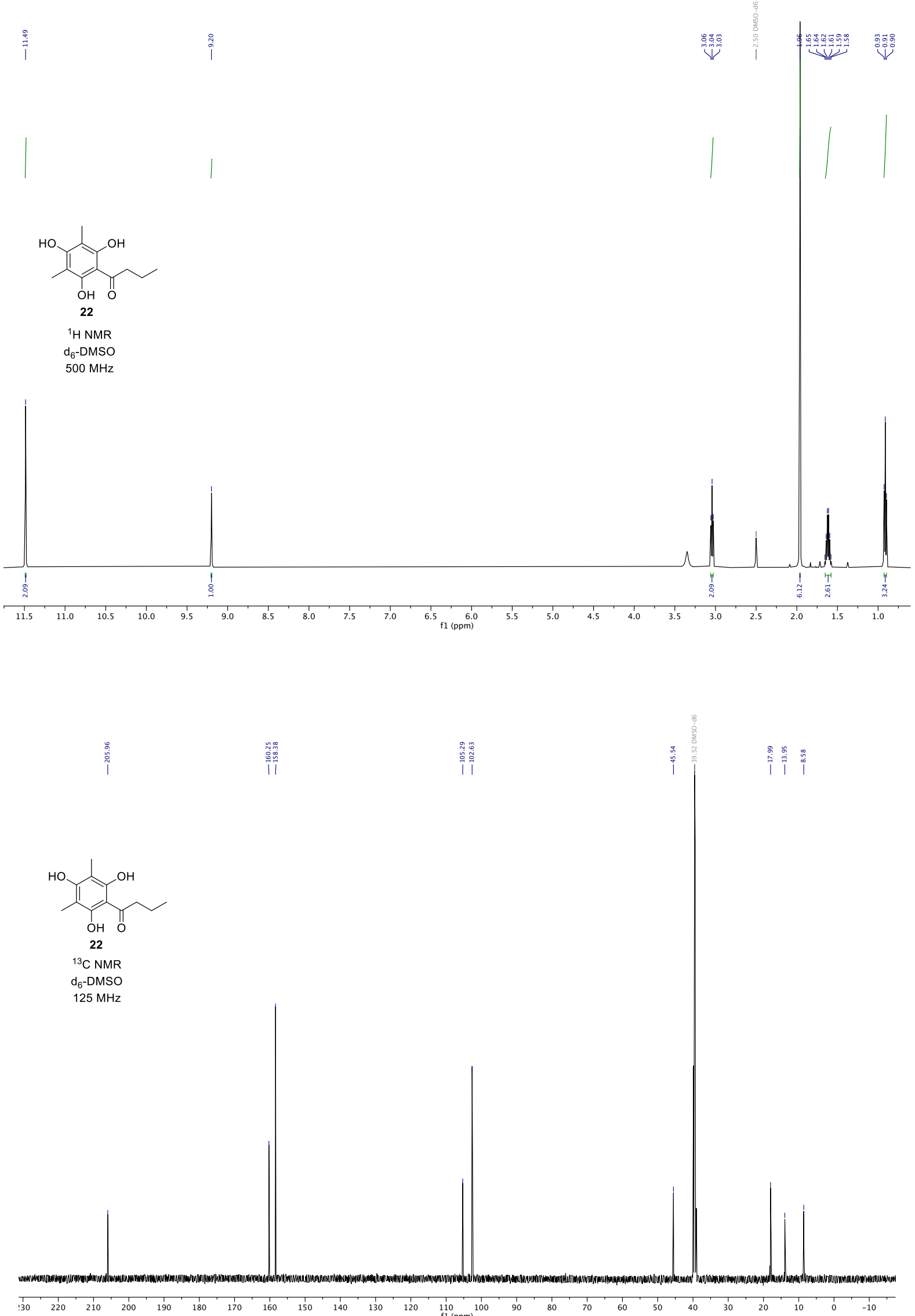

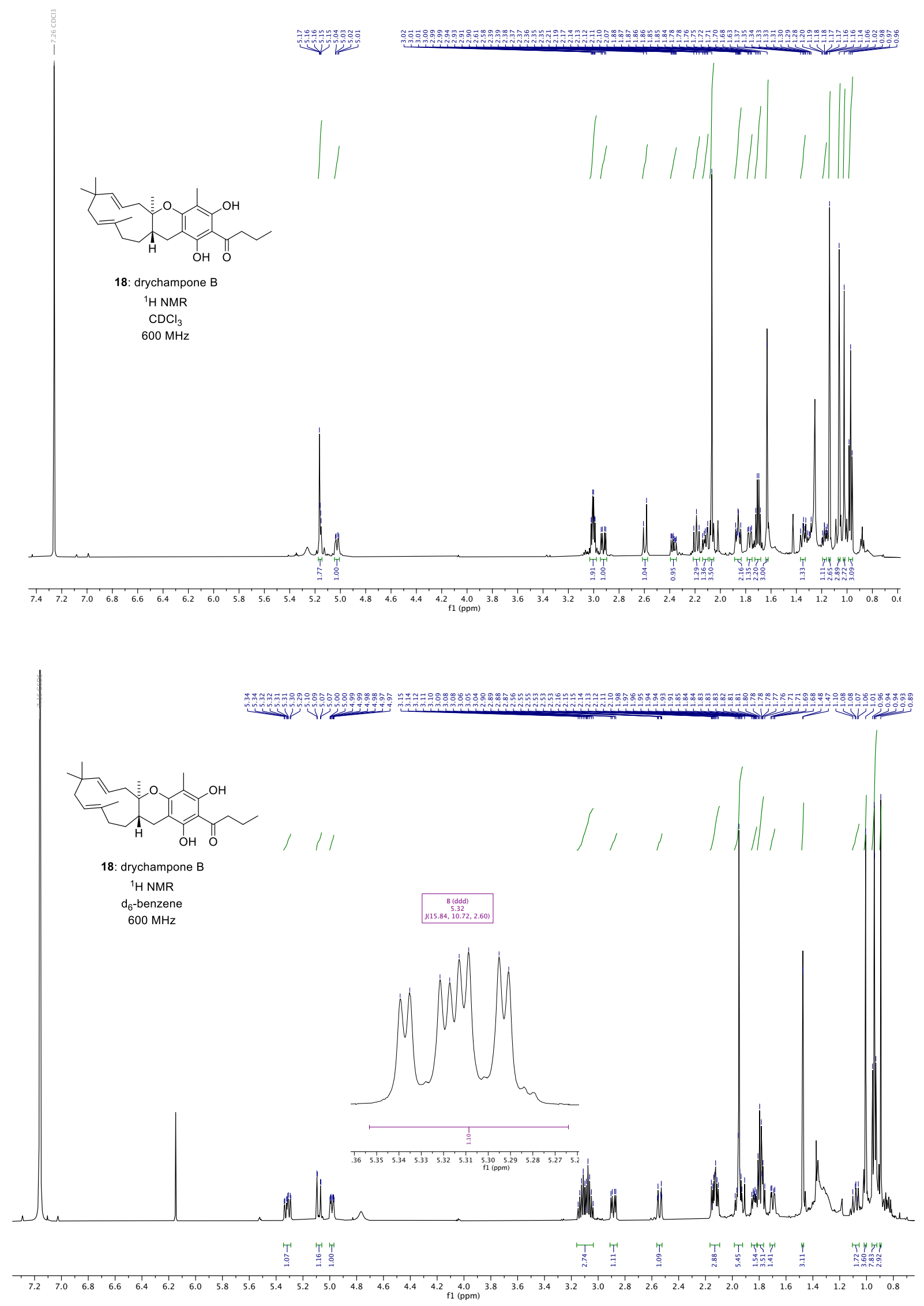


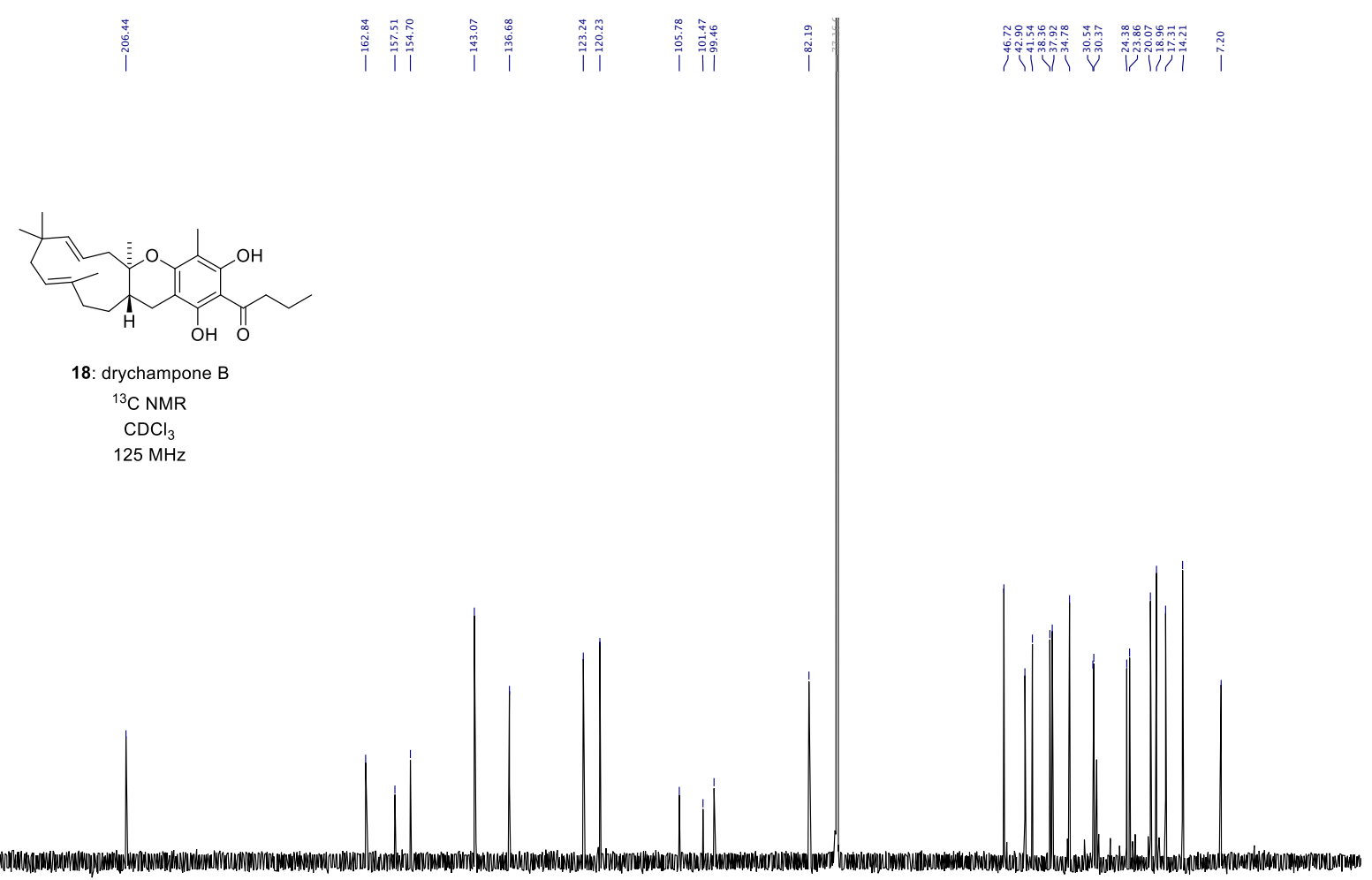

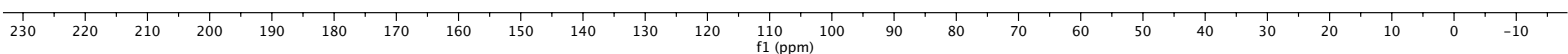

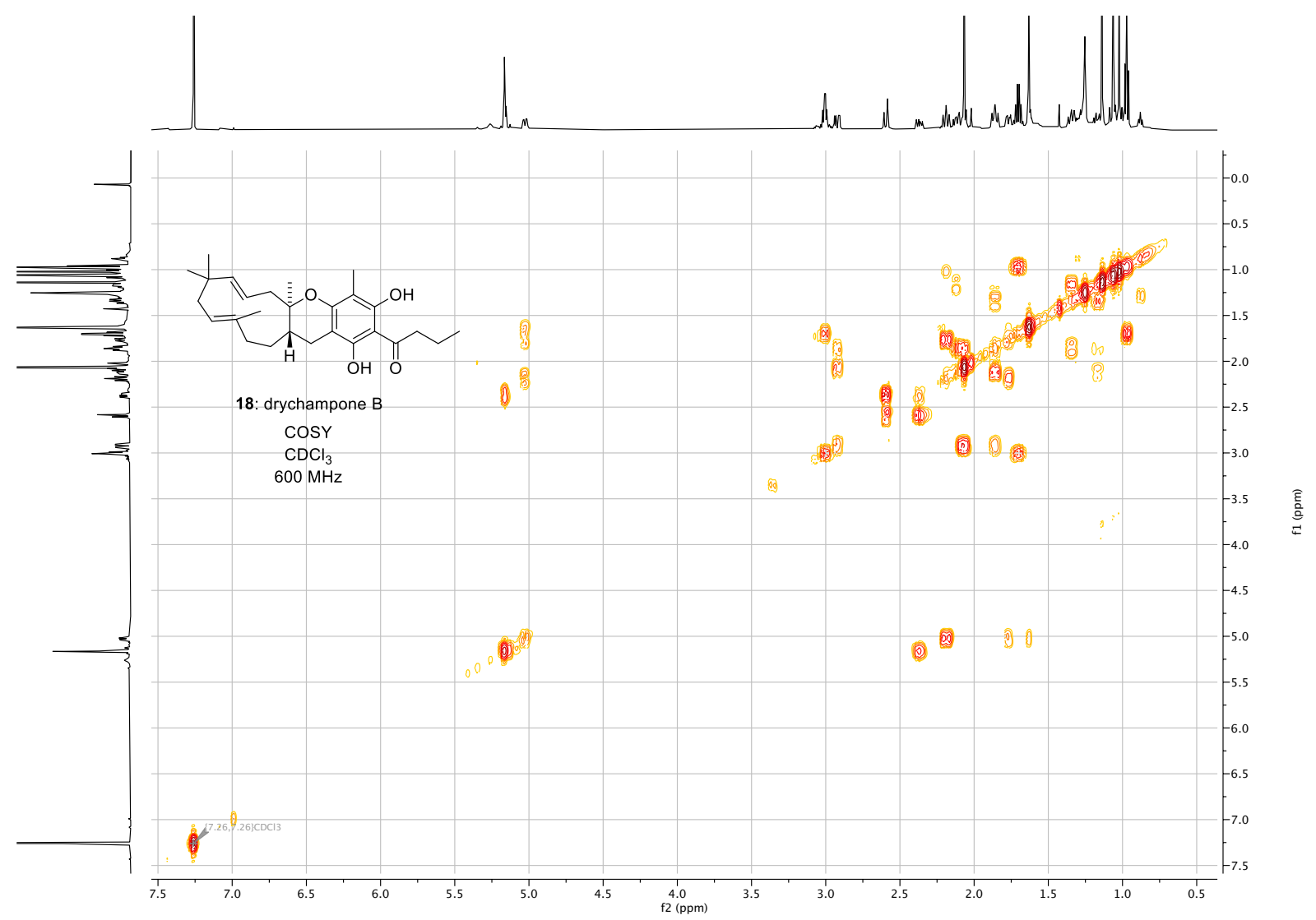




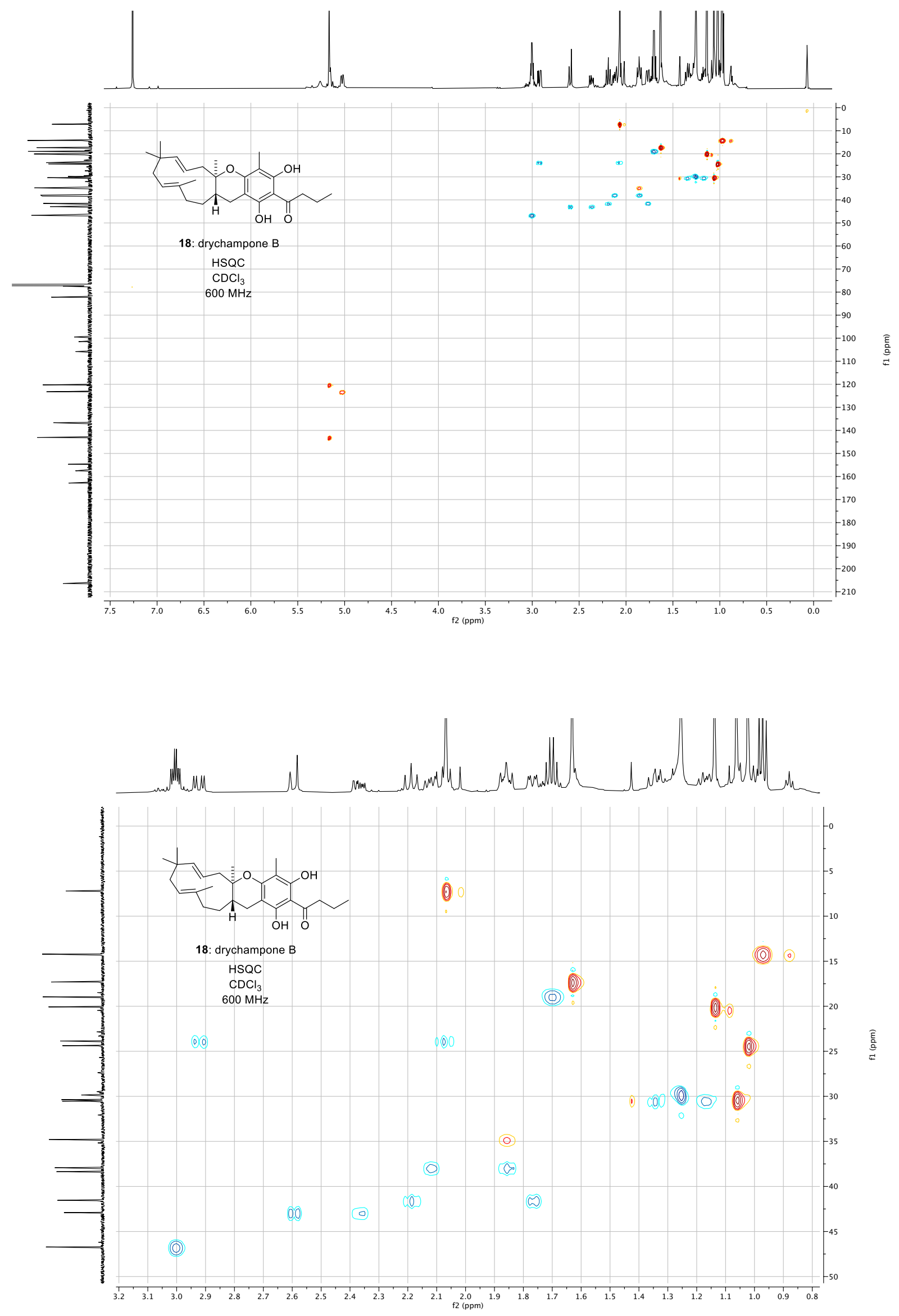




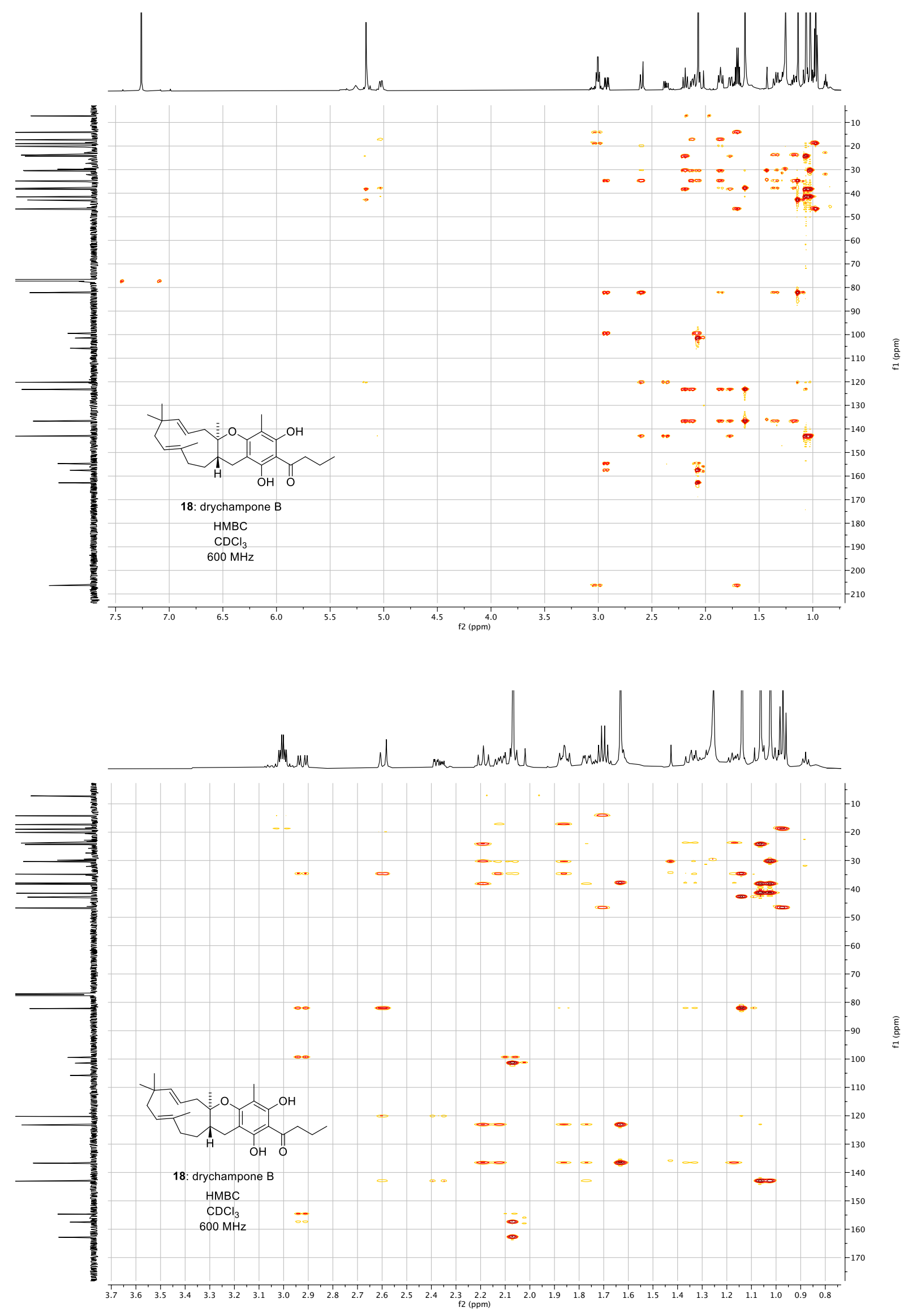




\section{Comparison of natural and synthetic NMR data}

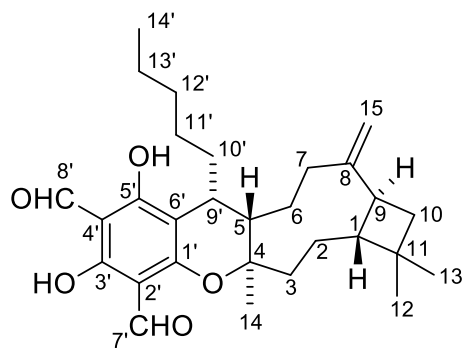

3: littordial C

Assignment

Isolated sample,

${ }^{1} \mathrm{H}$ NMR, $600 \mathrm{MHz}$,

Synthetic sample,

${ }^{1} \mathrm{H} \mathrm{NMR}, 600 \mathrm{MHz}$ $\mathrm{CDCl}_{3},(J, \mathrm{~Hz})$

Isolated sample,

${ }^{13} \mathrm{C}$ NMR, $150 \mathrm{MHz}$,

Synthetic sample

$\mathrm{CDCl}_{3,}(J, \mathrm{~Hz})$

1.37 , overlapped

$\mathrm{CDCl}_{3,}(\mathrm{~J}, \mathrm{~Hz})$

${ }^{13} \mathrm{C}$ NMR, $150 \mathrm{MHz}$

1.36 , overlapped

1.37 , overlapped

1.59 , overlapped

1.60 , overlapped

1.36 , overlapped

59.4

24.7

59.5

1.37 , overlapped

2.03, dd $(12.6,9.2)$

$2.03 \mathrm{dd}(12.6,10.8)$

1.74, overlapped

1.56 , overlapped

1.74 , overlapped

1.56 , overlapped

2.19, dd $(16.8,3.9)$

2.20, d (13.8)

$2.32, \operatorname{td}(13.1,11.9,3.3)$

2.33, ddd $(14.8,12.0,3.0)$

2.41, dd (18.0, 8.4)

2.41, q (8.7)

1.73 , overlapped

1.73, overlapped

47.7

24.8

$0.98, \mathrm{~s}$

$0.93, \mathrm{~s}$

$1.47, \mathrm{~s}$

$4.93, \mathrm{~s}$

$4.91, \mathrm{~s}$

$0.97, \mathrm{~s}$

$0.92, \mathrm{~s}$

$1.46, \mathrm{~s}$

$4.92, \mathrm{~s}$

88.1

47.8

40.6

88.2

36.1

40.7

35.5

36.1

150.8

35.6

$4.90, \mathrm{~s}$

42.4

150.9

23.5

42.5

34.7

23.6

21.7

34.8

21.8

29.5

29.7

23.2

23.3

111.0

111.2

163.9

164.0

105.7

167.9

105.8

104.2

168.0

167.4

104.3

111.1

167.5

10.00, s

9.99, s

192.1

111.3

$10.18, \mathrm{~s}$

$10.18, \mathrm{~s}$

191.9

192.2

2.93, m

192.0

2.93, d (10.2)

1.27 , overlapped

29.6

29.6

1.27 , overlapped

29.5

29.5

1.73 , overlapped

1.73 , overlapped

1.23 , overlapped

28.4

28.6

1.18 , overlapped

, overlapped

1.17 , overlapped

1.28 , overlapped

1.26 , overlapped

$0.85, \mathrm{t}(7.1)$

$13.25, \mathrm{~s}$

13.06, s

32.2

32.3

22.7

22.8

14.1

3.25 , 


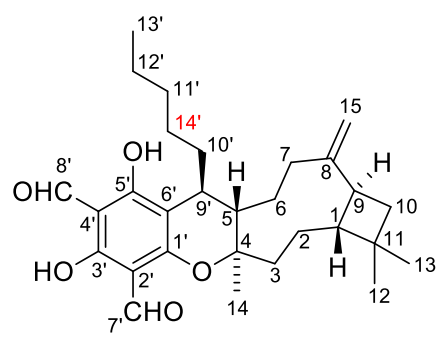

7: littordial E

revised structure

Assignment

$\begin{array}{cc}\text { Isolated sample, } & \text { Synthetic sample, } \\ { }^{1} \mathrm{H} \mathrm{NMR}, 600 \mathrm{MHz}, & { }^{1} \mathrm{H} \mathrm{NMR}, 600 \mathrm{MHz}, \\ \mathrm{CDCl}_{3},(J, \mathrm{~Hz}) & \mathrm{CDCl}_{3},(J, \mathrm{~Hz})\end{array}$
Isolated sample,
${ }^{13} \mathrm{C}$ NMR, $150 \mathrm{MHz}$,
$\mathrm{CDCl}_{3},(J, \mathrm{~Hz})$

Synthetic sample

$\mathrm{CDCl}_{3},(J, \mathrm{~Hz})$

,

2.00, overlapped
$.84, \operatorname{tdd}(13.8,5.4,3.0)$
95, ddd $(15.0,5.4,3.0)$
$2.10, \mathrm{~d}(2.4)$

2.01, ddd (11.8, 10.1 5.5)

1.44 , overlapped

53.2

53.4

1.84 , dddd $(15.1,12.6,5.3,3.1)$

3

4

5

6

7

8

9

10

11

12

13

14

15

$1^{\prime}$

$2^{\prime}$

$3^{\prime}$

$4^{\prime}$

$5^{\prime}$

$6^{\prime}$

$7^{\prime}$

$8^{\prime}$

$9^{\prime}$

$10^{\prime}$

$11^{\prime}$

$12^{\prime}$

$13^{\prime}$

$14^{\prime}$

$3^{\prime}-\mathrm{OH}$

5 '-OH
2.17, overlapped

$1.56, \mathrm{~m}$

1.70 , overlapped

2.13, m

2.52, dt (13.8, 5.4)

2.40, dd $(18.0,9.6)$

1.63 , dd $(10.8,7.8)$

1.69 , overlapped

$1.01, \mathrm{~s}$

$1.00, \mathrm{~s}$

$1.11, \mathrm{~s}$

4.94, s

4.90, s

10.02, s

10.17, s

$2.45, \mathrm{~m}$

1.42 , overlapped

2.18 , overlapped

$1.19, \mathrm{~m}$

2.03, overlapped

1.24, overlapped

$0.84, \mathrm{t}(7.2)$

$0.77, \mathrm{~m}$

1.00 , overlapped

13.3, s

$13.4, \mathrm{~s}$
1.95 , ddd $(15.4,5.6,3.1)$

2.13 , overlapped

2.17 , overlapped

1.56 , overlapped

1.70 , overlapped

2.14, overlapped

2.51, dt $(13.9,5.2)$

2.40, m

$1.63, \mathrm{dd}(10.7,7.7)$

1.71 , overlapped

$1.01, \mathrm{~s}$

$1.00, \mathrm{~s}$

1.12 , s

4.94, s

4.90, s

10.02, s

10.16, s

2.46, ddd (9.2, 5.0, 2.6)

1.43 , overlapped

2.18 , overlapped

1.14 , overlapped

1.20 , overlapped

1.22 , overlapped

$0.84, \mathrm{t}(7.1)$

$0.77, \mathrm{~m}$

1.02, overlapped

13.33, s

13.42, s
22.6

22.8

37.8

37.9

84.0

84.0

38.8

33.4

33.3

35.7

152.1

152.3

42.8

36.4

33.8

22.3

30.7

21.6

110.5

110.5

164.6

164.6

104.1

167.7

104.9

168.8

105.9

192.0

192.3

104.2

168.0

105.0

169.1

106.0

192.1

192.4

34.9

35.1

29.0

32.2

32.3

22.5

22.7

14.1

14.3

24.3

24.6 


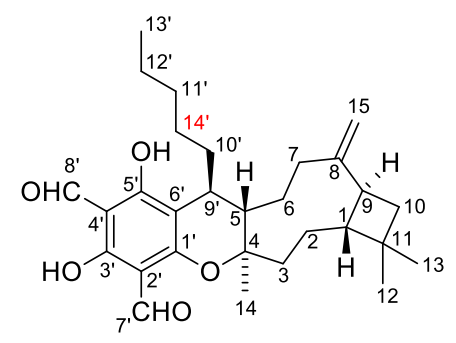

7: littordial E

revised structure

\begin{tabular}{|c|c|c|c|c|}
\hline Assignment & $\begin{array}{c}\text { Isolated sample, } \\
{ }^{1} \mathrm{H} \mathrm{NMR}, 600 \mathrm{MHz} \\
\mathrm{CDCl}_{3},(J, \mathrm{~Hz})\end{array}$ & $\begin{array}{l}\text { Synthetic sample, } \\
{ }^{1} \mathrm{H} \text { NMR, } 600 \mathrm{MHz} \text {, } \\
\mathrm{d}_{6} \text {-benzene, }(J, \mathrm{~Hz})\end{array}$ & $\begin{array}{c}\text { Isolated sample, } \\
{ }^{13} \mathrm{C} \mathrm{NMR}, 150 \mathrm{MHz} \\
\mathrm{CDCl}_{3,}(J, \mathrm{~Hz})\end{array}$ & $\begin{array}{c}\text { Synthetic sample } \\
{ }^{13} \mathrm{C} \mathrm{NMR}, 150 \mathrm{MHz}, \\
\mathrm{d}_{6} \text {-benzene }\end{array}$ \\
\hline 1 & 2.00 , overlapped & 2.04, ddd $(15.4,7.5,3.7)$ & 53.2 & 53.7 \\
\hline 2 & $1.84, \operatorname{tdd}(13.8,5.4,3.0)$ & $\begin{array}{l}1.21, \text { overlapped } \\
1.76, \text { overlapped }\end{array}$ & 22.6 & 22.8 \\
\hline 3 & $\begin{array}{c}1.95, \operatorname{ddd}(15.0,5.4,3.0) \\
2.10, \mathrm{~d}(2.4)\end{array}$ & $\begin{array}{l}1.60, \text { overlapped } \\
1.75, \text { overlapped }\end{array}$ & 37.8 & 37.8 \\
\hline 4 & & & 84.0 & 83.7 \\
\hline 5 & 2.17 , overlapped & $2.13, \operatorname{td}(9.1,2.8)$ & 38.7 & 39.0 \\
\hline 6 & $\begin{array}{c}1.56, \mathrm{~m} \\
1.70, \text { overlapped }\end{array}$ & $\begin{array}{l}1.21 \text {, overlapped } \\
1.44, \text { overlapped }\end{array}$ & 33.3 & 33.4 \\
\hline 7 & $\begin{array}{c}2.13, \mathrm{~m} \\
2.52, \mathrm{dt}(13.8,5.4)\end{array}$ & $\begin{array}{c}1.85, \operatorname{ddd}(13.5,11.3,4.8) \\
2.32, \mathrm{dt}(13.9,5.3)\end{array}$ & 35.6 & 35.8 \\
\hline 8 & & & 152.1 & 152.3 \\
\hline 9 & $2.40, \mathrm{dd}(18.0,9.6)$ & $2.21, \operatorname{td}(10.2,7.8)$ & 42.7 & 42.8 \\
\hline 10 & $\begin{array}{c}1.63, \mathrm{dd}(10.8,7.8) \\
1.69, \text { overlapped }\end{array}$ & $\begin{array}{l}1.59, \text { overlapped } \\
1.73, \text { overlapped }\end{array}$ & 36.2 & 36.6 \\
\hline 11 & & & 33.6 & 33.7 \\
\hline 12 & $1.01, \mathrm{~s}$ & $0.92(\mathrm{~s})$ & 22.2 & 22.3 \\
\hline 13 & $1.00, \mathrm{~s}$ & $0.99(\mathrm{~s})$ & 30.6 & 30.6 \\
\hline 14 & $1.11, \mathrm{~s}$ & $0.76(\mathrm{~s})$ & 21.5 & 21.2 \\
\hline 15 & $\begin{array}{l}4.94, \mathrm{~s} \\
4.90, \mathrm{~s}\end{array}$ & $\begin{array}{l}4.87(\mathrm{~s}) \\
5.00(\mathrm{~s})\end{array}$ & 110.5 & 110.7 \\
\hline $1^{\prime}$ & & & 164.6 & 164.4 \\
\hline $2^{\prime}$ & & & 104.1 & 104.6 \\
\hline $3^{\prime}$ & & & 167.7 & 168.1 \\
\hline $4^{\prime}$ & & & 104.9 & 105.3 \\
\hline $5^{\prime}$ & & & 168.8 & 169.4 \\
\hline $6^{\prime}$ & & & 105.9 & 106.0 \\
\hline $7^{\prime}$ & $10.02, \mathrm{~s}$ & $9.98, \mathrm{~s}$ & 192.0 & 191.8 \\
\hline $8^{\prime}$ & 10.17, s & $10.10, \mathrm{~s}$ & 192.3 & 192.0 \\
\hline $9^{\prime}$ & $2.45, \mathrm{~m}$ & $2.45, \mathrm{~m}$ & 34.9 & 35.4 \\
\hline $10^{\prime}$ & $\begin{array}{l}1.42, \text { overlapped } \\
2.18, \text { overlapped }\end{array}$ & $\begin{array}{c}\text { 1.46, overlapped } \\
2.40, \mathrm{~m}\end{array}$ & 28.8 & 29.4 \\
\hline $11^{\prime}$ & $\begin{array}{c}1.19, \mathrm{~m} \\
2.03, \text { overlapped }\end{array}$ & $\begin{array}{l}1.28, \text { overlapped } \\
1.60, \text { overlapped }\end{array}$ & 32.2 & 32.6 \\
\hline $12^{\prime}$ & 1.24 , overlapped & $\begin{array}{l}1.27, \text { overlapped } \\
1.42, \text { overlapped }\end{array}$ & 22.5 & 23.1 \\
\hline $13^{\prime}$ & $0.84, \mathrm{t}(7.2)$ & $0.86, \mathrm{t}(7.0)$ & 14.1 & 14.4 \\
\hline $14^{\prime}$ & $\begin{array}{c}0.77, \mathrm{~m} \\
1.00, \text { overlapped }\end{array}$ & $\begin{array}{l}1.02, \text { overlapped } \\
1.20, \text { overlapped }\end{array}$ & 24.3 & 24.9 \\
\hline $\begin{array}{l}3^{\prime}-\mathrm{OH} \\
5^{\prime}-\mathrm{OH}\end{array}$ & $\begin{array}{l}13.3, \mathrm{~s} \\
13.4, \mathrm{~s}\end{array}$ & & & \\
\hline
\end{tabular}




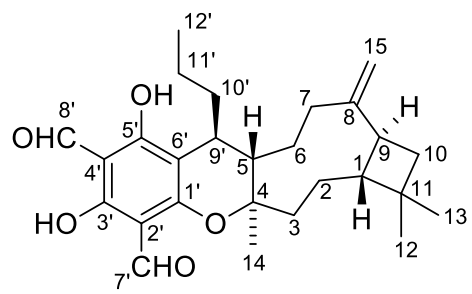

8: littordial $\mathrm{F}$

revised structure

\begin{tabular}{|c|c|c|c|c|}
\hline Assignment & $\begin{array}{c}\text { Isolated Sample, } \\
{ }^{1} \mathrm{H} \mathrm{NMR}, 600 \mathrm{MHz} \\
\mathrm{CDCl}_{3},(J, \mathrm{~Hz})\end{array}$ & $\begin{array}{c}\text { Synthetic Sample, } \\
{ }^{1} \mathrm{H} \mathrm{NMR}, 600 \mathrm{MHz} \\
\mathrm{CDCl}_{3},(J, \mathrm{~Hz})\end{array}$ & $\begin{array}{c}\text { Isolated Sample, } \\
{ }^{13} \mathrm{C} \mathrm{NMR}, 150 \mathrm{MHz}, \\
\mathrm{CDCl}_{3,}(J, \mathrm{~Hz})\end{array}$ & $\begin{array}{c}\text { Synthetic Sample, } \\
{ }^{13} \mathrm{C} \mathrm{NMR}, 150 \mathrm{MHz}, \\
\mathrm{CDCl}_{3},(J, \mathrm{~Hz})\end{array}$ \\
\hline 1 & $2.00, \mathrm{~m}$ & 1.98, overlapped & 53.5 & 53.6 \\
\hline 2 & $\begin{array}{l}1.46, \mathrm{~m} \\
1.86, \mathrm{~m}\end{array}$ & $\begin{array}{c}\text { 1.43, overlapped } \\
1.84, \text { dddd }(15.1,12.2,5.3,3.0)\end{array}$ & 22.6 & 22.7 \\
\hline 3 & $1.97, \mathrm{~m}$ & $\begin{array}{l}1.95 \text {, ddd }(15.4,5.7,3.0) \\
2.12, \text { overlapped }\end{array}$ & 37.9 & 38.1 \\
\hline 4 & & & 84.0 & 84.1 \\
\hline 5 & 2.18 , overlapped & 2.16 , overlapped & 38.9 & 39.1 \\
\hline 6 & $\begin{array}{l}1.60, \text { overlapped } \\
2.18 \text {, overlapped }\end{array}$ & $\begin{array}{l}1.57 \text {, overlapped } \\
1.69 \text {, overlapped }\end{array}$ & 33.7 & 33.2 \\
\hline 7 & $\begin{array}{l}1.74 \text {, overlapped } \\
2.54 \text {, overlapped }\end{array}$ & $\begin{array}{l}2.14 \text {, overlapped } \\
2.51 \text {, overlapped }\end{array}$ & 35.6 & 35.8 \\
\hline 8 & & & 154.0 & 152.4 \\
\hline 9 & 2.43 , overlapped & $2.41, \mathrm{~m}$ & 42.5 & 42.7 \\
\hline 10 & 1.65, overlapped & $\begin{array}{l}1.63, \mathrm{dd}(10.7,7.7) \\
1.72, \text { overlapped }\end{array}$ & 36.3 & 36.4 \\
\hline 11 & & & 33.1 & 33.8 \\
\hline 12 & $1.04, \mathrm{~s}$ & $1.00, \mathrm{~s}$ & 22.2 & 22.3 \\
\hline 13 & $1.02, \mathrm{~s}$ & $1.01, \mathrm{~s}$ & 30.6 & 30.7 \\
\hline 14 & $1.14, \mathrm{~s}$ & $1.12, \mathrm{~s}$ & 21.3 & 21.5 \\
\hline 15 & $\begin{array}{l}4.92, \mathrm{~s} \\
4.97, \mathrm{~s}\end{array}$ & $\begin{array}{l}4.90, \mathrm{~s} \\
4.95, \mathrm{~s}\end{array}$ & 110.3 & 110.5 \\
\hline $1^{\prime}$ & & & 164.5 & 164.6 \\
\hline $2^{\prime}$ & & & 104.9 & 105.1 \\
\hline $3^{\prime}$ & & & 167.8 & 168.0 \\
\hline $4^{\prime}$ & & & 104.0 & 104.2 \\
\hline $5^{\prime}$ & & & 169.0 & 169.2 \\
\hline $6^{\prime}$ & & & 106.0 & 106.1 \\
\hline $7^{\prime}$ & & 10.01 & 192.2 & 192.3 \\
\hline $8^{\prime}$ & & 10.16 & 192.0 & 192.1 \\
\hline $9^{\prime}$ & $\begin{array}{l}2.14, \mathrm{~m} \\
2.48, \mathrm{~m}\end{array}$ & $2.47, \mathrm{~m}$ & 35.0 & 35.2 \\
\hline $10^{\prime}$ & $1.44, \mathrm{~m}$ & $\begin{array}{l}1.43 \text {, overlapped } \\
2.16 \text {, overlapped }\end{array}$ & 31.6 & 31.7 \\
\hline $11^{\prime}$ & $\begin{array}{l}0.88, \mathrm{~m} \\
1.05, \mathrm{~m}\end{array}$ & $\begin{array}{l}0.83 \text {, overlapped } \\
1.04 \text {, overlapped }\end{array}$ & 18.3 & 18.4 \\
\hline $12^{\prime}$ & $0.83, \mathrm{~d}(3.6)$ & $0.81, \mathrm{~m}$ & 14.5 & 14.6 \\
\hline $3^{\prime}-\mathrm{OH}$ & $13.3, \mathrm{~s}$ & $13.32, \mathrm{~s}$ & & \\
\hline $5^{\prime}-\mathrm{OH}$ & $13.4, \mathrm{~s}$ & $13.42, \mathrm{~s}$ & & \\
\hline
\end{tabular}




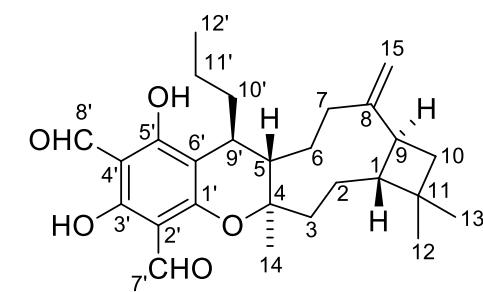

8: littordial $\mathrm{F}$ revised structure

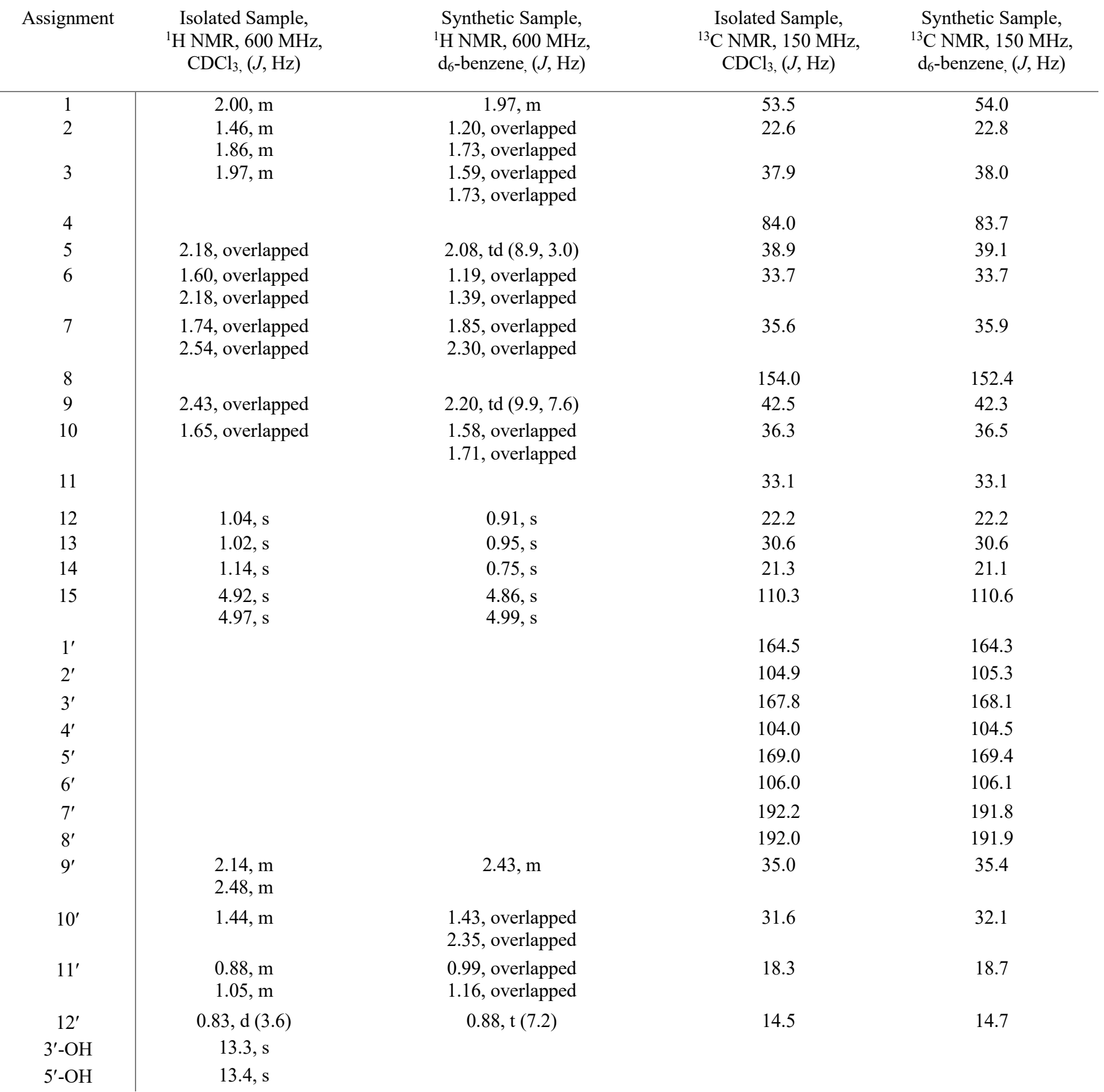




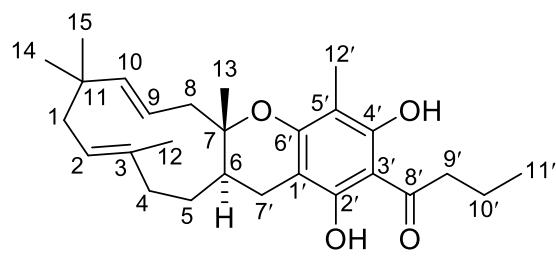

18: drychampone B revised structure

Assignment

3

4

5

6

7

8

9

10

11

12

13

14

15

$1^{\prime}$

$2^{\prime}$

$3^{\prime}$

$4^{\prime}$

$5^{\prime}$

$6^{\prime}$

$7^{\prime}$

$8^{\prime}$

$9^{\prime}$

$10^{\prime}$

$11^{\prime}$

$12^{\prime}$
Isolated sample ${ }^{1} \mathrm{H} \mathrm{NMR}, 500 \mathrm{MHz}$, $\mathrm{CDCl}_{3,}(J, \mathrm{~Hz})$
Synthetic sample ${ }^{1} \mathrm{H}$ NMR, $600 \mathrm{MHz}$, $\mathrm{CDCl}_{3,}(J, \mathrm{~Hz})$
Isolated sample ${ }^{13} \mathrm{C}$ NMR, $125 \mathrm{MHz}$, $\mathrm{CDCl}_{3},(J, \mathrm{~Hz})$
Synthetic sample ${ }^{13} \mathrm{C}$ NMR, $150 \mathrm{MHz}$ $\mathrm{CDCl}_{3,}(J, \mathrm{~Hz})$
$1.74, \mathrm{dd}(12.5,4.2)$

2.17, t (12.5)

$5.00, \mathrm{dd},(8.7,4.2)$

1.84

2.10, m

$1.16, \mathrm{~m}$

$1.28, \mathrm{~m}$

1.83

$2.35, \mathrm{~m}$

2.56, d (14.5)

5.15, m

5.14, d (16.2)

$1.61, \mathrm{~s}$

$1.12, \mathrm{~s}$

$1.00, \mathrm{~s}$

$1.04, \mathrm{~s}$

$$
\begin{gathered}
1.77, \mathrm{dd}(12.9,4.4) \\
2.19, \mathrm{t}(12.4) \\
5.03, \mathrm{~m}
\end{gathered}
$$$$
41.6
$$

123.3

136.8

38.0

89 - 1.82, overlapped

$2.16-2.09, \mathrm{~m}$

1.21- $1.15, \mathrm{~m}$

$1.34, \mathrm{~m}$

$1.89-1.82$, overlapped

2.37, ddd (14.5, 7.1, 3.0)

2.60, d (14.5)

5.18-5.14, m

5.18-5.14, m

$1.63, \mathrm{~s}$

$1.14, \mathrm{~s}$

$1.02, \mathrm{~s}$

$1.06, \mathrm{~s}$

2,07 , overlapped

2.92, dd $(16.1,5.7)$

2.90, dd $(16.8,5.2)$

$$
\begin{gathered}
2.99, \operatorname{td}(7.5,3.0) \\
1.67, \mathrm{~m} \\
0.95, \mathrm{t}(7.5) \\
2.04, \mathrm{~s}
\end{gathered}
$$

$$
\begin{gathered}
3.00, \mathrm{td}(7.4,3.8) \\
1.70, \mathrm{q}(7.4) \\
0.97, \mathrm{t}(7.4) \\
2.07, \mathrm{~s}
\end{gathered}
$$

41.5

123.2

136.7

37.9

30.5

34.8

82.2

42.9

120.2

143.1

38.4

17.3

20.1

24.4

30.4

99.5

154.7

105.8

162.8

101.5

157.5

23.9

206.4

46.7

19.0

14.2

7.2 


\section{CD spectra}

Littordial C
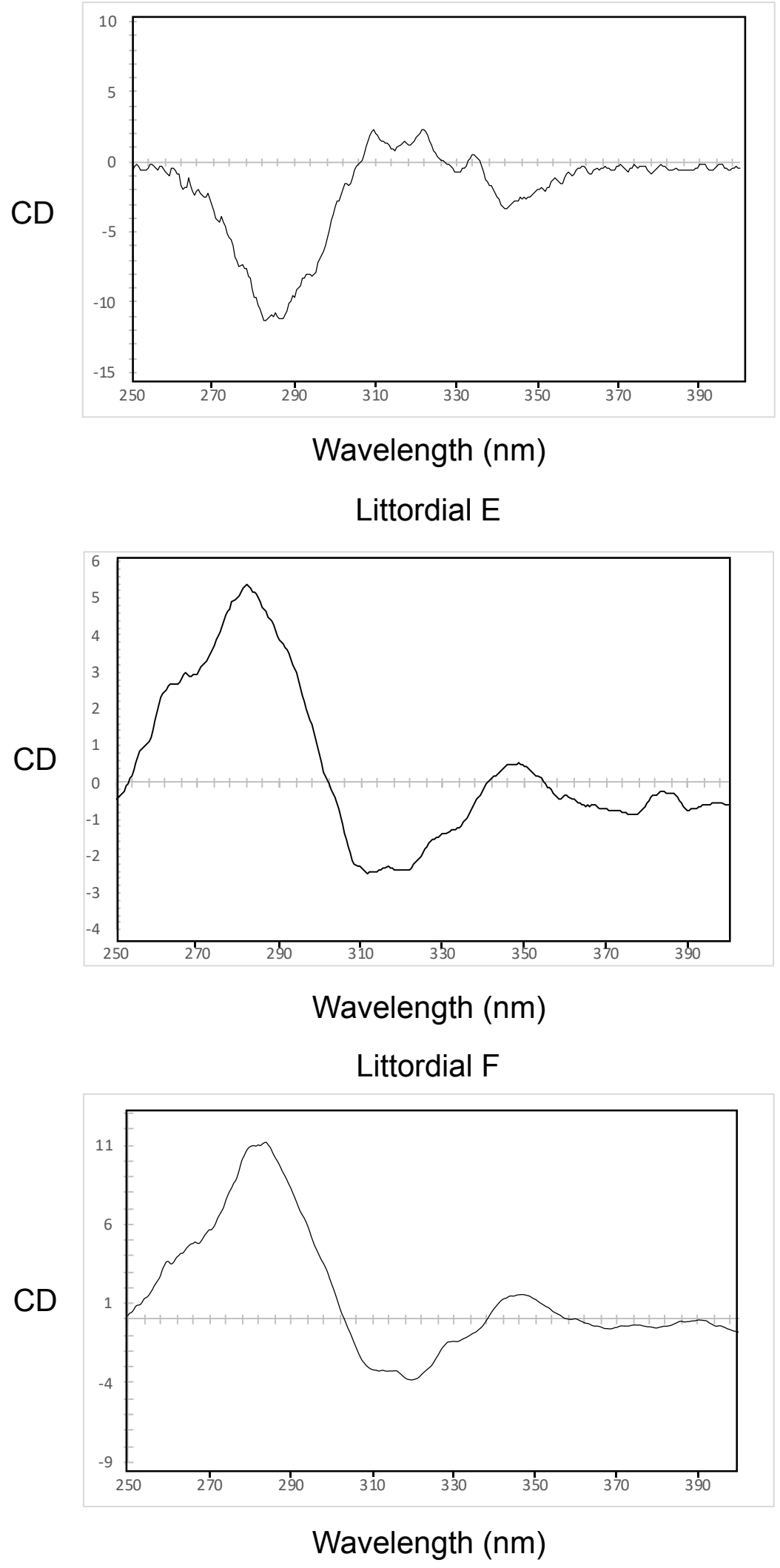


\section{Single crystal X-ray data}

To obtain single crystals suitable for X-ray analysis, littordial C (20 mg, $43 \mu \mathrm{mol})$ was dissolved in ethanol $(0.5 \mathrm{~mL})$ in a $5 \mathrm{~mL}$ vial and left at room temperature overnight to afford colourless prisms (10 mg, $50 \%$ ). A single crystal of littordial $\mathrm{C}$ was mounted in paratone-N oil on a MiteGen crystal mount and the data was collected on an X-ray diffraction at 150(2) K on an Oxford X-calibur single crystal diffractometer using Mo K $\alpha$ radiation. The data set was corrected for absorption using a multi-scan method, and the structures solved by direct methods using SHELXS-974 and refined by full-matrix least squares on F2 by SHELXL-2015, ${ }^{5}$ interfaced through the programs X-Seed ${ }^{6}$ and Olex2. ${ }^{7}$ Except as otherwise indicated, all nonhydrogen atoms were refined anisotropically and hydrogen atoms were included as invariants at geometrically estimated positions. Full data for the structure determination have been deposited with the Cambridge Crystallographic Data Centre as CCDC 2017518. Copies of this information may be obtained free of charge from The Director, CCDC, 12 Union Street, Cambridge CB2 1EZ, U.K. (fax, +44-1223-336-033; e-mail, deposit@ccdc.cam.ac.uk). The table below provides the crystal data and structure refinement details for littordial C.

\footnotetext{
${ }^{4}$ Sheldrick, G. M. Phase annealing in SHELX-90: direct methods for larger structures. Acta Crystallogr. Sect. A 46, 467-473 (1990).

${ }^{5}$ Sheldrick, G. M. A short history of SHELX. Acta Crystallogr. Sect. A 64, 112-122 (2008).

${ }^{6}$ Barbour, L. J. X-seed - A software tool for supramolecular crystallography. J. Supramol. Chem. 1, 189-191 (2001).

${ }^{7}$ Bourhis, L. J., Dolomanov, O. V., Gildea, R. J., Howard, J. A. K. \& Puschmann, H. The anatomy of a comprehensive constrained, restrained refinement program for the modern computing environment Olex2 dissected. Acta Crystallogr. Sect. A 71, 59-75 (2015).
} 


\begin{tabular}{|l|l|}
\hline \multicolumn{2}{|l|}{ Crystal data and structure refinement for littordial C } \\
\hline Identification code & littordial C \\
\hline Empirical formula & $\mathrm{C}_{29} \mathrm{H}_{40} \mathrm{O}_{5}$ \\
\hline Formula weight & 468.61 \\
\hline Temperature/K & $149.9(3)$ \\
\hline Crystal system & tetragonal \\
\hline Space group & $\mathrm{P}_{1} 2_{1} 2$ \\
\hline $\mathrm{a} / \AA$ & $11.13120(10)$ \\
\hline $\mathrm{b} / \AA$ & $11.13120(10)$ \\
\hline $\mathrm{c} / \AA$ & $41.3856(7)$ \\
\hline$\alpha{ }^{\circ}$ & 90 \\
\hline$\beta /{ }^{\circ}$ & 90 \\
\hline$\gamma /{ }^{\circ}$ & 90 \\
\hline Volume/ $\AA^{3}$ & $5127.83(13)$ \\
\hline $\mathrm{Z}$ & 8 \\
\hline$\rho_{\text {calcg/cm }}^{3}$ & 1.214 \\
\hline$\mu / \mathrm{mm}^{-1}$ & 0.081 \\
\hline F $(000)$ & 2032.0 \\
\hline Crystal size/mm ${ }^{3}$ & $0.28 \times 0.253 \times 0.049$ \\
\hline Radiation & MoK $\alpha(\lambda=0.71073)$ \\
\hline $2 \Theta$ range for data collection/ & 6.504 to 58.868 \\
\hline Index ranges & $-15 \leq \mathrm{h} \leq 15,-15 \leq \mathrm{k} \leq 15,-56 \leq 1 \leq 57$ \\
\hline Reflections collected & 183383 \\
\hline Independent reflections & $6837\left[\mathrm{R}_{\text {int }}=0.0878, \mathrm{R}_{\text {sigma }}=0.0419\right]$ \\
\hline Data/restraints/parameters & $6837 / 0 / 316$ \\
\hline Goodness-of-fit on $\mathrm{F}^{2}$ & 1.061 \\
\hline Final $\mathrm{R}$ indexes $[\mathrm{I}>=2 \sigma(\mathrm{I})]$ & $\mathrm{R}_{1}=0.0522, \mathrm{wR} \mathrm{R}_{2}=0.1013$ \\
\hline Final $\mathrm{R}$ indexes [all data] & $\mathrm{R}_{1}=0.0738, \mathrm{wR} \mathrm{R}_{2}=0.1076$ \\
\hline Largest diff. peak/hole $/ \mathrm{e} \AA^{-3}$ & $0.30 /-0.20$ \\
\hline Flack parameter & $0.3(3)$ \\
\hline
\end{tabular}




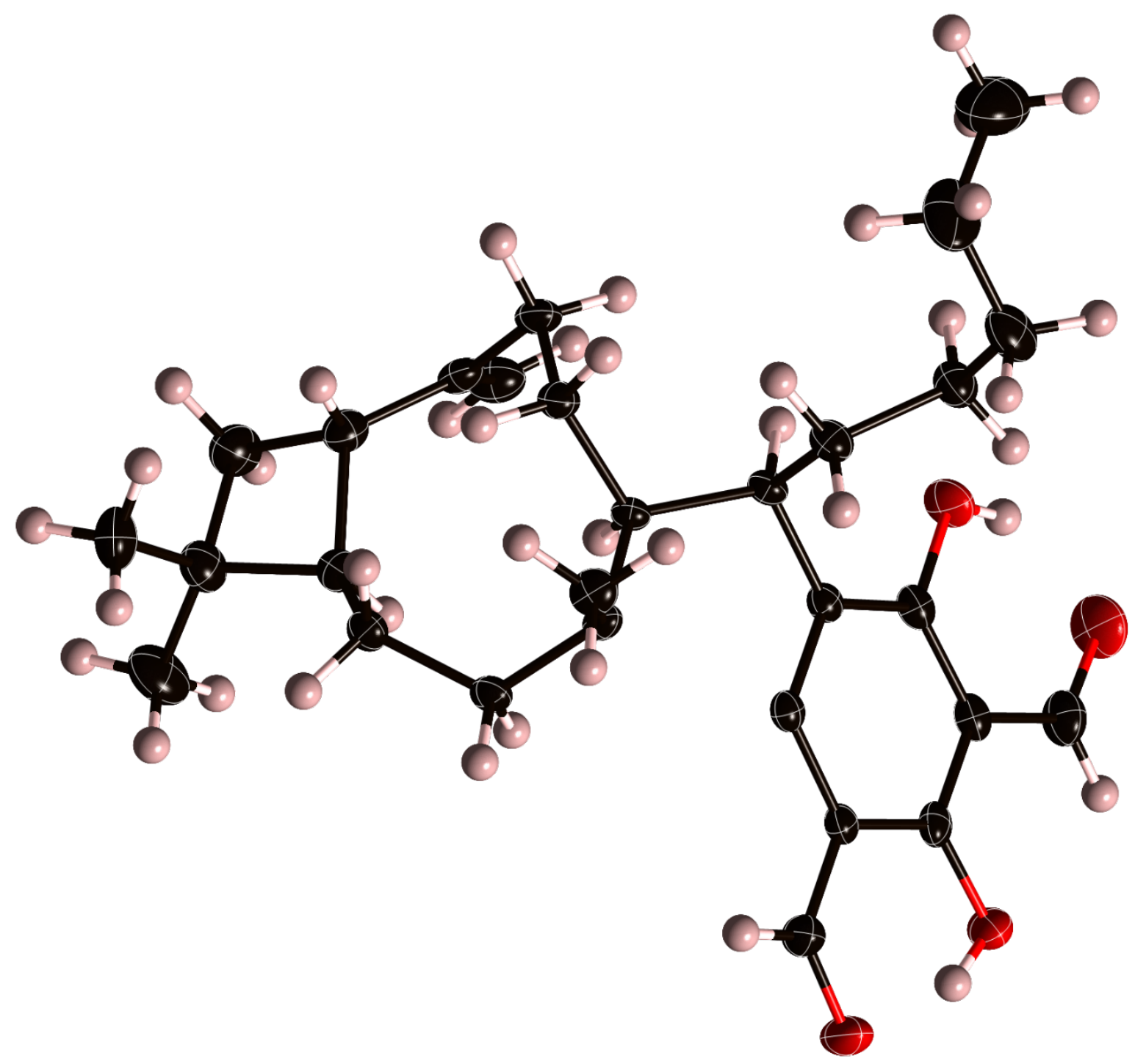

A perspective view of one selected molecule of the asymmetric unit of littordial $\mathrm{C}$, with all non-hydrogen atoms represented by ellipsoids at the $50 \%$ probability level (Carbon, black; Hydrogen, white; Oxygen, red). 


\section{Computational methods}

Calculations were carried out with density functional theory (DFT) using Gaussian 16 (revision A.03). ${ }^{8}$ Geometries were optimised in the gas phase at the M06-2X/6-31+G(d) level. ${ }^{9}$ Stable ground states and transition states were identified by the number of imaginary vibrational frequencies. Zero-point energies and thermal corrections. The transition states were shown to connect reactants and products using intrinsic reaction coordinate calculations. ${ }^{10}$ Single-point energy calculations of the optimised geometries were carried out in the gas phase at the M06$2 \mathrm{X} / 6-311+\mathrm{G}(\mathrm{d}, \mathrm{p})$ level and in toluene solvent using the SMD continuum solvent model ${ }^{11}$ using parameters adapted for hexafluoroisopropanol, ${ }^{12}$ at the M06-2X/6-311+G(d,p) level. The optimised geometries are given below in Cartesian coordinates and the energies given in units of Hartree. The Gibbs free energy in solution at $298.15 \mathrm{~K}$ was calculated as $G_{\text {soln }}=E_{\mathrm{M} 06-2 \mathrm{X} / 6 \text { - }}$ $311+\mathrm{G}(\mathrm{d}, \mathrm{p})$,sol. $+T C G_{\mathrm{M} 06-2 \mathrm{X} / 6-31+\mathrm{G}(\mathrm{d})}+k_{\mathrm{B}} \mathrm{T} \ln \left(\mathrm{c} k_{\mathrm{B}} \mathrm{T} / p\right)$. TCG is the thermal correction to the free energy. The final term is a correction to account for the difference in translational entropy resulting from the change in concentration from ideal gas-phase species at pressure $p=1 \mathrm{~atm}$ to ideal solution-phase species at concentration $\mathrm{c}=1 \mathrm{~mol} / \mathrm{L}$ at temperature $\mathrm{T}$ and is equal to $7.93 \mathrm{~kJ} / \mathrm{mol}$ at $298.15 \mathrm{~K}$.

\footnotetext{
${ }^{8}$ M. J. Frisch, G. W. Trucks, H. B. Schlegel, G. E. Scuseria, M. A. Robb, J. R. Cheeseman, G. Scalmani, V. Barone, G. A. Petersson, H. Nakatsuji, X. Li, M. Caricato, A. V. Marenich, J. Bloino, B. G. Janesko, R. Gomperts, B. Mennucci, H. P. Hratchian, J. V. Ortiz, A. F. Izmaylov, J. L. Sonnenberg, D. Williams-Young, F. Ding, F. Lipparini, F. Egidi, J. Goings, B. Peng, A. Petrone, T. Henderson, D. Ranasinghe, V. G. Zakrzewski, J. Gao, N. Rega, G. Zheng, W. Liang, M. Hada, M. Ehara, K. Toyota, R. Fukuda, J. Hasegawa, M. Ishida, T. Nakajima, Y. Honda, O. Kitao, H. Nakai, T. Vreven, K. Throssell, J. A. Montgomery, Jr., J. E. Peralta, F. Ogliaro, M. J. Bearpark, J. J. Heyd, E. N. Brothers, K. N. Kudin, V. N. Staroverov, T. A. Keith, R. Kobayashi, J. Normand, K. Raghavachari, A. P. Rendell, J. C. Burant, S. S. Iyengar, J. Tomasi, M. Cossi, J. M. Millam, M. Klene, C. Adamo, R. Cammi, J. W. Ochterski, R. L. Martin, K. Morokuma, O. Farkas, J. B. Foresman, and D. J. Fox, Gaussian, Inc., Wallingford CT, 2016. ${ }^{9}$ Y. Zhao, D. Truhlar, Theor. Chem. Acc. 2008, 120, 215.

${ }^{10}$ H. P. Hratchian, H. B. Schlegel, J. Chem. Phys. 2004, 120, 9918.

${ }^{11}$ A. V. Marenich, C. J. Cramer, D. G. Truhlar, J. Phys. Chem. B 2009, 113, 6378.

${ }^{12}$ E. Richmond, J. Yi, V. D. Vuković, F. Sajadi, C. N. Rowley, J. Moran, Chem. Sci. 2018, 9, 6411.
} 
Table 1. Calculated free energies of starting materials and products. ${ }^{a}$ Relative free energy refers to the comparison of isomers for ground state structures, and activation barriers for transition state structures.

\begin{tabular}{|c|c|c|c|c|c|c|}
\hline Structure & $\begin{array}{l}\text { E(M06-2X/6- } \\
31+G(d)) / \text { a.u. }\end{array}$ & TCG/a.u. & $\begin{array}{l}\text { E(M06-2X/6- } \\
311+G(d, p)) / a . u .\end{array}$ & $\begin{array}{l}\text { E(M06-2X/6- } \\
311+G(d, p), \\
\text { smd=HFIP)/a.u. }\end{array}$ & $\begin{array}{l}\text { G(M06-2X/6- } \\
\text { 311+G(d,p), } \\
\text { smd=HFIP, } \\
\text { kBTln(ckBT/p } \\
\text { )/kJ.mol }{ }^{-1}\end{array}$ & $\begin{array}{l}\text { Relative free } \\
\text { energy } \\
\left(\mathrm{kJ} . \mathrm{mol}^{-1}\right)^{\mathrm{a}}\end{array}$ \\
\hline caryophyllene- $\alpha \alpha$ & -585.7257137 & 0.319369 & -585.8701592 & -585.8837733 & -1537391.4 & 0.2 \\
\hline caryophyllene- $\beta \alpha$ & -585.7243348 & 0.318311 & -585.8688689 & -585.8828104 & -1537391.7 & 0.0 \\
\hline caryophyllene- $\beta \beta$ & -585.7241116 & 0.318325 & -585.8685618 & -585.8824629 & -1537390.7 & 0.9 \\
\hline caryophyllene- $\alpha \beta$ & -585.7176052 & 0.31845 & -585.8620886 & -585.8758407 & -1537373.0 & 18.7 \\
\hline$E-0-Q M$ & -840.2913633 & 0.181864 & -840.517878 & -840.5314837 & -2206330.0 & 0.0 \\
\hline$Z$-o-QM & -840.2928995 & 0.182755 & -840.5197473 & -840.5319208 & -2206328.8 & 1.2 \\
\hline caryophyllene- $\alpha \alpha$-E-oQM-DA-endo-TS & -1426.013502 & 0.526059 & -1426.384883 & -1426.415368 & -3743664.5 & 57.0 \\
\hline caryophyllene- $\alpha \alpha$-E-oQM-DA-exo-TS & -1426.005817 & 0.527022 & -1426.377629 & -1426.408544 & -3743644.0 & 77.4 \\
\hline caryophyllene- $\alpha \alpha$-Z-oQM-DA-endo-TS & -1426.013397 & 0.525987 & -1426.385137 & -1426.415652 & -3743665.4 & 54.8 \\
\hline caryophyllene- $\alpha \alpha$-Z-oQM-DA-exo-TS & -1426.010993 & 0.525416 & -1426.383138 & -1426.41439 & -3743663.6 & 56.6 \\
\hline caryophyllene- $\beta \alpha$-E-oQM-DA-endo-TS & -1426.014362 & 0.524671 & -1426.385792 & -1426.416022 & -3743669.8 & 51.8 \\
\hline caryophyllene- $\beta \alpha$-E-oQM-DA-exo-TS & -1426.009367 & 0.52547 & -1426.380946 & -1426.4119 & -3743656.9 & 64.8 \\
\hline caryophyllene- $\beta \alpha$-Z-oQM-DA-endo-TS & -1426.01598 & 0.526132 & -1426.387605 & -1426.417901 & -3743670.9 & 49.6 \\
\hline caryophyllene- $\beta \alpha$-Z-oQM-DA-exo-TS & -1426.011766 & 0.526119 & -1426.383722 & -1426.415246 & -3743664.0 & 56.5 \\
\hline caryophyllene- $\beta \beta$-E-oQM-DA-endo-TS & -1426.013817 & 0.525369 & -1426.385293 & -1426.414933 & -3743665.1 & 55.6 \\
\hline caryophyllene- $\beta \beta$-E-oQM-DA-exo-TS & -1426.008401 & 0.525517 & -1426.379909 & -1426.411371 & -3743655.4 & 65.3 \\
\hline caryophyllene- $\beta \beta$-Z-oQM-DA-endo-TS & -1426.013723 & 0.525687 & -1426.385407 & -1426.414787 & -3743663.9 & 55.6 \\
\hline caryophyllene- $\beta \beta$-Z-oQM-DA-exo-TS & -1426.014931 & 0.523749 & -1426.38694 & -1426.418456 & -3743678.6 & 40.9 \\
\hline littordial A $\alpha$ & -1426.090088 & 0.534693 & -1426.458849 & -1426.480928 & -3743813.9 & 0.0 \\
\hline littordial A $\beta$ & -1426.089845 & 0.53431 & -1426.45846 & -1426.480299 & -3743813.3 & 0.6 \\
\hline littordial B $\alpha$ & -1426.087814 & 0.534646 & -1426.455989 & -1426.477299 & -3743804.5 & 16.1 \\
\hline littordial B $\beta$ & -1426.092068 & 0.534246 & -1426.46053 & -1426.483029 & -3743820.6 & 0.0 \\
\hline littordial F $\alpha$ & -1426.089128 & 0.534153 & -1426.457787 & -1426.479869 & -3743812.5 & 5.6 \\
\hline littordial $\mathbf{F} \boldsymbol{\beta}$ & -1426.091118 & 0.533955 & -1426.459633 & -1426.481796 & -3743818.1 & 0.0 \\
\hline littordial $\mathrm{Z} \alpha$ & -1426.055952 & 0.535309 & -1426.45999 & -1426.481311 & -3743813.3 & 6.8 \\
\hline littordial $\mathrm{Z} \beta$ & -1426.058801 & 0.53523 & -1426.462887 & -1426.483839 & -3743820.1 & 0.0 \\
\hline littordial C $\alpha$ & -1504.637837 & 0.589259 & -1505.062961 & -1505.086141 & -3950048.6 & 17.2 \\
\hline littordial $C \beta$ & -1504.641707 & 0.588419 & -1505.067514 & -1505.091867 & -3950065.9 & 0.0 \\
\hline littordial $\mathbf{E} \alpha$ & -1504.676253 & 0.587463 & -1505.064759 & -1505.088862 & -3950060.5 & 6.0 \\
\hline littordial $\mathrm{E} \beta$ & -1504.678204 & 0.587124 & -1505.066487 & -1505.090796 & -3950066.5 & 0.0 \\
\hline
\end{tabular}




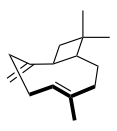

$\alpha \alpha$

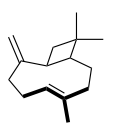

$\beta \alpha$

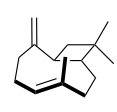

$\beta \beta$

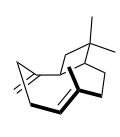

$\alpha \beta$

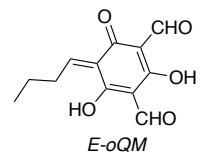

rel. $\Delta \mathrm{G}=0 \mathrm{kj} \cdot \mathrm{mol}^{-1}$

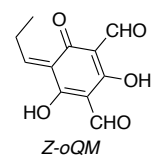

rel. $\Delta \mathrm{G}=1.2 \mathrm{kj} \cdot \mathrm{mol}^{-1}$

rel. $\Delta \mathrm{G}=0.2 \mathrm{kj} \cdot \mathrm{mol}^{-1} \quad$ rel. $\Delta \mathrm{G}=0 \mathrm{kj} \cdot \mathrm{mol}^{-1} \quad$ rel. $\Delta \mathrm{G}=0.9 \mathrm{kj} \cdot \mathrm{mol}^{-1}$ rel. $\Delta \mathrm{G}=18.7 \mathrm{kj} \cdot \mathrm{mol}^{-1}$
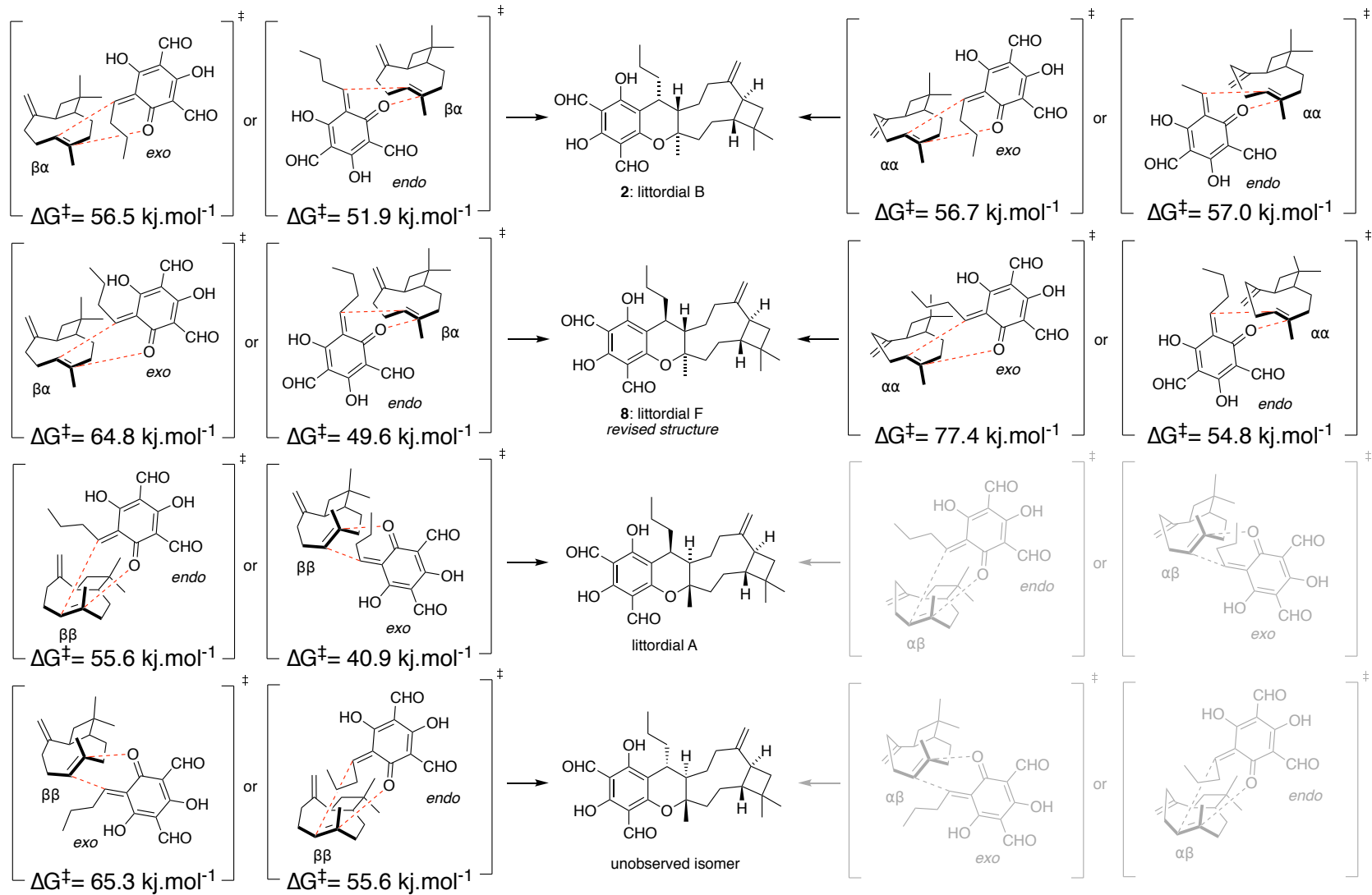

$\Delta \mathrm{G}^{\ddagger}=40.9 \mathrm{kj} \cdot \mathrm{mol}^{-1}$

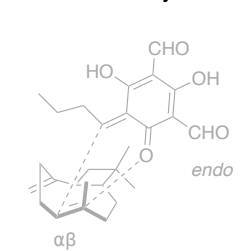

$\Delta \mathrm{G}^{\ddagger}=54.8 \mathrm{kj} \cdot \mathrm{mol}^{-1}$
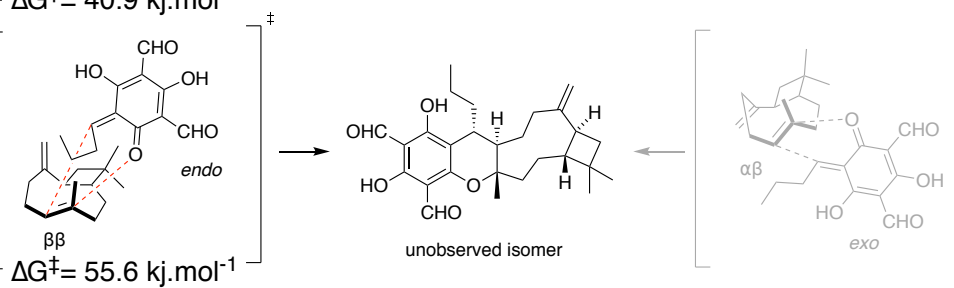

Calculated activation free energies of the cycloadditions of caryopyllene with o-QM 16 (M06-2X/6-311+G(d,p)//M06-2X/6-31+G(d), smd = hexafluoroisopropanol, 298K)

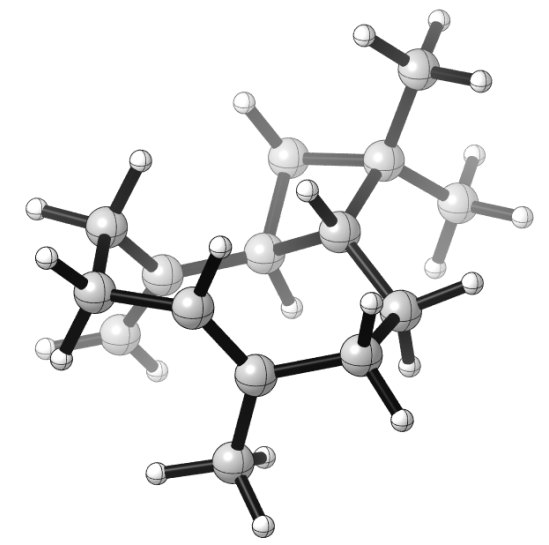

caryophyllene- $\alpha \alpha$

$\mathrm{E}_{(\mathrm{M} 06-2 \mathrm{X} / 6-31+\mathrm{G}(\mathrm{d})):-585.7257137}$

TCG $(\mathrm{M} 06-2 \mathrm{X} / 6-31+\mathrm{G}(\mathrm{d})): 0.319369$

$\mathrm{E}_{(\mathrm{M} 06-2 \mathrm{X} / 6-311+\mathrm{G}(\mathrm{d}, \mathrm{p})):-585.8701592}$

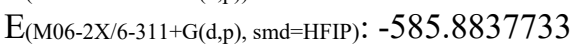

Imaginary frequencies: none 

C $2.35765800 \quad 1.28920400-1.27276900$
H $2.750474002 .24433300-1.64359300$
H $1.60131300 \quad 0.94806400-1.99172900$
H $3.16746900 \quad 0.55706500-1.27840400$
C $1.752024001 .48368400 \quad 0.09197200$
C $2.00209500 \quad 0.72477700 \quad 1.16689500$
H $1.48650900 \quad 0.99305700 \quad 2.09185500$
C $0.61554400 \quad 2.47146500 \quad 0.16406200$
H $0.44692100 \quad 2.76399100 \quad 1.20764200$
$\mathrm{H} \quad 0.82340500 \quad 3.38593000-0.40608700$
C $-0.67678900 \quad 1.80794600-0.37586600$
$\mathrm{H}-0.64372100 \quad 1.76121100-1.47302300$
$\mathrm{H}-1.52680800 \quad 2.45745900-0.12391500$
C $-0.93160000 \quad 0.40743700 \quad 0.18316800$
$\mathrm{H}-0.58765100 \quad 0.35821400 \quad 1.22583200$
C $-0.44091400-0.86235600-0.57765700$
H $-0.55666300-0.67585300-1.65374400$
C $-2.37367500-0.18484000 \quad 0.14935000$
C $-1.72081500-1.57264600-0.06539000$
$\mathrm{H}-1.53510900-2.07596600 \quad 0.89197400$
$\mathrm{H}-2.21006300-2.27453300-0.74988300$
C $-3.18432300 \quad 0.24956700-1.07140300$
$\mathrm{H}-4.09662400-0.35403400-1.14837500$
$\mathrm{H}-2.62542400 \quad 0.12830900-2.00581300$
$\mathrm{H}-3.48563400 \quad 1.30114700-0.99547900$
C $-3.19056700 \quad 0.00443200 \quad 1.41882000$
$\mathrm{H}-3.43782800 \quad 1.06345800 \quad 1.56961800$
$\mathrm{H}-2.63609400-0.34122300 \quad 2.29891400$
$\mathrm{H}-4.13310000-0.55488400 \quad 1.36995200$
C $0.88279200-1.56453900-0.38038600$
C $1.48818800-2.13057100-1.43033200$
H $1.08834400-2.04013700-2.43782900$
H $2.40837900-2.70128600-1.31822500$
C $2.64341200-0.63079000 \quad 1.19322500$
H $3.14617600 \quad-0.81271700 \quad 2.15024700$
H $3.38697100 \quad-0.75649500 \quad 0.39954400$
C $1.52051400-1.68181400 \quad 0.99361500$
H $0.76654200-1.553795001 .78211700$
H $1.94095900-2.687057001 .11458000$

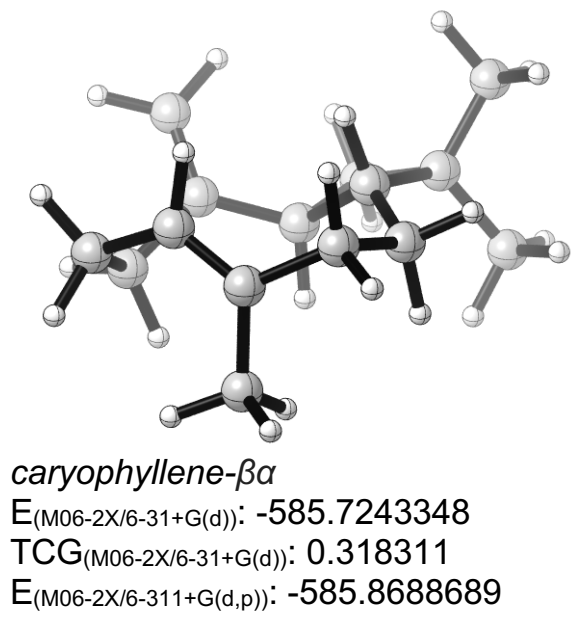

$E_{(M 06-2 X / 6-311+G(d, p), \text { smd=HFIP): }-585.8828104}$ Imaginary frequencies: none 
H $3.12871200-1.326708001 .44095100$

C $1.84581800-1.41663600-0.30945700$

C $2.30204900-0.30592400-0.89676800$

H $1.93275200-0.07688600-1.89649100$

C $0.72789700-2.19037100-0.95508100$

H $0.69214700-1.96709400-2.02855800$

H $0.87160000-3.27395200-0.84745800$

C $-0.61531800-1.79571000-0.31369800$

$\mathrm{H}-0.62501000-2.111210000 .73825500$

$\mathrm{H}-1.41508500-2.36887500-0.80582900$

C $-0.98657200-0.31483900-0.40198700$

$\mathrm{H}-0.838994000 .04546400-1.43182200$

C -0.419503000 .774931000 .58201000$

$\mathrm{H}-0.156999000 .292576001 .53342500$

C - 2.428379000 .057399000 .06962300

C - 1.851491001 .361085000 .66445700

$\mathrm{H}-1.984475002 .20945000-0.01309200$

$\mathrm{H}-2.204308001 .652772001 .66011500$

C - $2.96781400-0.853034001 .17244000$

$\mathrm{H}-3.88991500-0.425334001 .58404500$

H -2.25579000 -0.95979800 1.99904700

$\mathrm{H}-3.20161800-1.855924000 .79626200$

C $-3.456698000 .21133400-1.04175300$

$\mathrm{H}-3.66495200-0.75558700-1.51812800$

$\mathrm{H}-3.096690000 .89872200-1.81588200$

H $-4.404060000 .60417600-0.65217000$

C 0.703440001 .714346000 .18062200

C $0.521322002 .70648700-0.69723500$

$\mathrm{H}-0.429146002 .89075800-1.19041600$

H $1.339739003 .37058800-0.96784700$

C $3.049311000 .79965300-0.19930800$

H $3.406494001 .52590900-0.93681100$

H 3.931309000 .436327000 .34243900

C 2.085365001 .504069000 .78643900

H 2.509931002 .467458001 .09317800

H 1.993985000 .892323001 .69195400

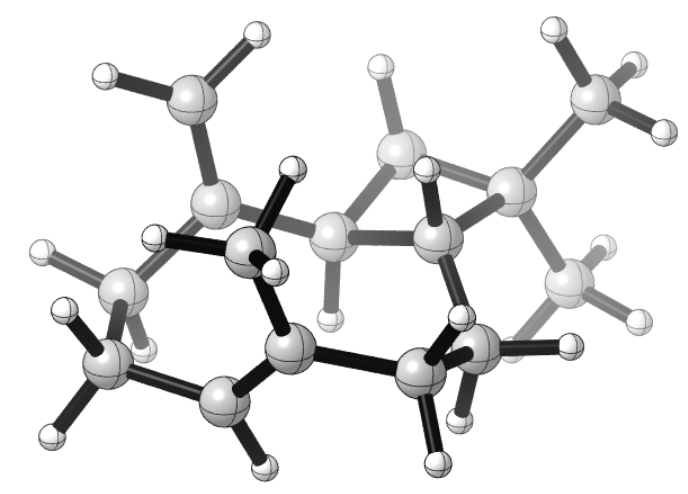

caryophyllene- $\beta \beta$

$\mathrm{E}_{(\mathrm{MO6}-2 \times / 6-31+G(d)):}-585.7241116$

$\mathrm{TCG}_{(\mathrm{M} 06-2 \times / 6-31+\mathrm{G}(\mathrm{d}))}: 0.318325$

$E_{(M 06-2 X / 6-311+G(d, p)):}-585.8685618$ 
$E_{(M 06-2 X / 6-311+G(d, p) \text {, smd=HFIP): }-585.8824629}$

Imaginary frequencies: none

C 2.286183001 .311355001 .52130700

H 1.487077000 .960834002 .18829600

H 2.60516600 2.29431800 1.89072100

H 3.126219000 .621670001 .61889000

C 1.770990001 .438179000 .11204500

C $2.156269000 .67358200-0.91603800$

H $1.695327000 .86333200-1.88937500$

C $0.632055002 .42681800-0.06774800$

H 0.430119002 .914742000 .89422300

H $0.905062003 .22612600-0.76861300$

C - $0.654482001 .73201400-0.55279000$

$\mathrm{H}-0.589632001 .54223200-1.63268200$

$\mathrm{H}-1.496113002 .42689600-0.41786900$

C - 0.980022000 .418249000 .15880800

$\mathrm{H}-0.727206000 .493562001 .22820100$

C $-0.44734100-0.95049300-0.38083600$

$\mathrm{H}-0.38239300-0.88116100-1.47438100$

C - $2.42975500-0.143496000 .03902600$

C - $1.81606600-1.562979000 .01165200$

$\mathrm{H}-1.77837100-2.004683001 .01342400$

$\mathrm{H}-2.25447100-2.29089500-0.68035400$

C -3.10281200 0.19210700 -1.29216700

$\mathrm{H}-4.02425100-0.39287200-1.39872300$

$\mathrm{H}-2.46112100-0.04084800-2.14957500$

H -3.37230100 $1.25315200-1.35219200$

C -3.358886000 .183333001 .19862000$

$\mathrm{H}-3.582807001 .257727001 .22850400$

H - $2.90403100-0.093892002 .15664900$

H $-4.31093700-0.354071001 .10658600$

C $0.81736100-1.599434000 .14501200$

C $0.93446800-1.979343001 .42077100$

H $0.13966800-1.825254002 .14781900$

H $1.84338400-2.449754001 .78983100$

C $2.94897400-0.59707200-0.82693000$

H $3.54157700-0.628395000 .09173000$

H $3.64612000-0.70025100-1.66722700$

C $1.97079800-1.79974600-0.82389200$

H $2.52479400-2.71031400-0.56793200$

H $1.57271600-1.94063600-1.83698900$ 


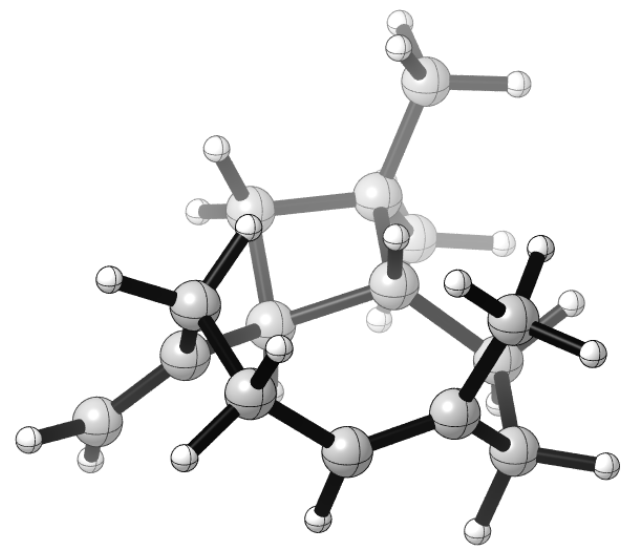

caryophyllene- $\alpha \beta$

$E_{(M 06-2 X / 6-31+G(d)):-585.7176052}$

$\mathrm{TCG}_{(\mathrm{M} 06-2 \mathrm{X} / 6-31+G(\mathrm{~d}))}: 0.31845$

$E_{(M 06-2 X / 6-311+G(d, p)):}-585.8620886$

$E_{(M 06-2 X / 6-311+G(d, p), s m d=H F I P):}-585.8758407$ Imaginary frequencies: none

C - $1.73996400-2.336284001 .04352000$

$\mathrm{H}-0.71621200-2.505867001 .40323500$

H - $2.18377400-3.327564000 .88594200$

$\mathrm{H}-2.30201100-1.838168001 .83790500$

C $-1.74144900-1.56122200-0.24618700$

C - $2.35062200-0.38715600-0.44019900$

$\mathrm{H}-2.254108000 .05259300-1.43319700$

C $-0.78877500-2.03383900-1.31423600$

$\mathrm{H}-0.82239800-3.12177300-1.45627400$

$\mathrm{H}-1.06881000-1.57348300-2.26761700$

C $0.66480500-1.61010100-0.95062900$

H $1.22543800-1.45975600-1.88141100$

H $1.16524100-2.44026900-0.43052500$

C $0.82148600-0.38276000-0.04710600$

H $0.30867600-0.571975000 .90404600$

C $0.512352001 .08665200-0.47509000$

H $0.743426001 .21985200-1.53977400$

C 2.272886000 .060286000 .31466400

C 1.739306001 .508651000 .38068200

H 1.427073001 .775454001 .39846100

H 2.37193300 2.30952100 -0.01817500

C $3.27244000-0.11061700-0.82784400$

H $4.209126000 .40435300-0.58307600$

H $2.898563000 .30906800-1.76866800$

H $3.50576800-1.16807300-1.00033100$

C $2.83584400-0.524960001 .60217200$

H $3.01872300-1.602279001 .49673500$

H 2.13992100 -0.37939300 2.43666200

H $3.78813700-0.049646001 .86830700$

C $-0.766026001 .83911100-0.16065100$

C - $1.111327002 .89396700-0.90623200$

$\mathrm{H}-0.541681003 .18145000-1.78719200$

$\mathrm{H}-1.974496003 .50828400-0.65783200$

C - 2.841108000 .576329000 .60047600 
H -3.27519400 0.084377001 .47697100

$\mathrm{H}-3.610905001 .232104000 .17828100$

C -1.62220100 1.441149001 .03215000

$\mathrm{H}-1.024867000 .866853001 .75116900$

H - 1.973453002 .339098001 .55341500

\section{E-O-QM}

$E_{(M 06-2 X / 6-31+G(d)):}-840.2913633$

$T C G_{(M 06-2 X / 6-31+G(d)): 0} 0.181864$

$E_{(M 06-2 X / 6-311+G(d, p)):}-840.517878$

$E_{(M 06-2 X / 6-311+G(d, p), \text { smd=HFIP): }-840.5314837}$

Imaginary frequencies: none

C $0.56465800-0.47515600-0.00004700$

C $1.85161500-0.893136000 .00000100$

C $0.165936000 .92499200-0.00007100$

C - $1.174210001 .30597100-0.00000500$

C - 2.191857000 .284357000 .00000200

C - $1.89334400-1.075345000 .00003500$

C - $0.51022100-1.53526200-0.00003700$

C - $2.96671500-2.041142000 .00001700$

$\mathrm{H}-2.67722900-3.10102900-0.00004900$

C - 1.526221002 .710346000 .00005500

$\mathrm{H}-2.594279002 .965293000 .00014900$

O - $4.16290300-1.726526000 .00003400$

O -0.681241003 .611048000 .00002100$

O - $0.21190700-2.72226500-0.00009800$

O $1.125443001 .82265600-0.00016000$

H $0.692710002 .73025200-0.00013500$

O -3.43741300 0.714947000 .00006400

H -4.03454500 -0.09420300 0.00007200

C $5.64921100-0.154322000 .00004300$

H 5.699759000 .490170000 .88452900

H $6.53566600-0.794780000 .00034500$

C $4.36696300-0.981905000 .00009700$

H $4.34668100-1.637779000 .87951000$

H $4.34669500-1.63789600-0.87923300$

C $3.11875500-0.099002000 .00002300$

H $3.122854000 .57484400-0.86794900$

H 3.122797000 .574915000 .86792900

H $5.700001000 .48966900-0.88479000$

H $1.95563200-1.980716000 .00006700$ 


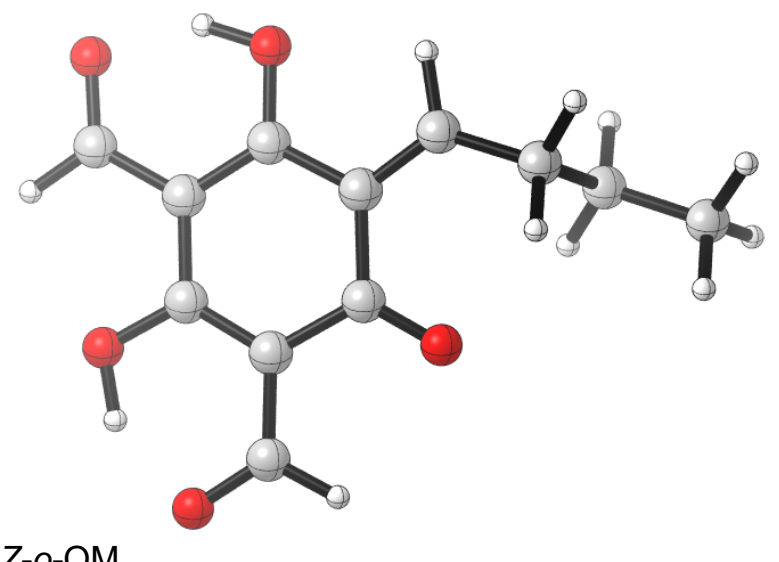

Z-o-QM

$\mathrm{E}_{(\mathrm{MO6}-2 \times / 6-31+G(d)):}-840.2928995$

$\mathrm{TCG}_{(\mathrm{M} 06-2 \times / 6-31+G(\mathrm{~d})): 0.182755}$

$E_{(M 06-2 X / 6-311+G(d, p)):}-840.5197473$

$E_{(M 06-2 X / 6-311+G(d, p), \text { smd }=H F I P):}-840.5319208$

Imaginary frequencies: none

C $0.62238900-0.58613400-0.23360500$

C $1.79886200-1.21294200-0.48361900$

C - $0.58017000-1.40277300-0.06997000$

C - $1.84416100-0.834688000 .05463700$

C - 1.966590000 .601063000 .03909200

C - $0.866129001 .44614100-0.07287400$

C $0.486334000 .90635500-0.16341400$

C - $1.061933002 .87659500-0.05859700$

$\mathrm{H}-0.159928003 .49934200-0.13418300$

C -3.01190700 -1.68243900 0.19334600

$\mathrm{H}-3.98780300-1.187272000 .28545900$

O -2.174824003 .408889000 .03045200$

O $-2.94548000-2.914321000 .20957300$

O $1.475761001 .63142800-0.18071300$

O $-0.41903500-2.70703900-0.06019300$

$\mathrm{H}-1.32300600-3.128472000 .04341100$

O -3.191513001 .075794000 .14628700$

$\mathrm{H}-3.127504002 .078817000 .12723700$

C 5.187625000 .346742000 .45925700

H $5.081450001 .30174700-0.06561100$

H 5.677293000 .540820001 .41815600

C $3.82301500-0.304796000 .66472800$

H 3.174127000 .358843001 .24424200

H $3.93057500-1.239781001 .22792100$

C $3.13864400-0.59430800-0.68993200$

H $3.048426000 .34821000-1.23540600$

H $3.76458200-1.28641900-1.26402900$

H $1.75797100-2.30125800-0.51152300$

H $5.84866100-0.29548600-0.13321300$ 


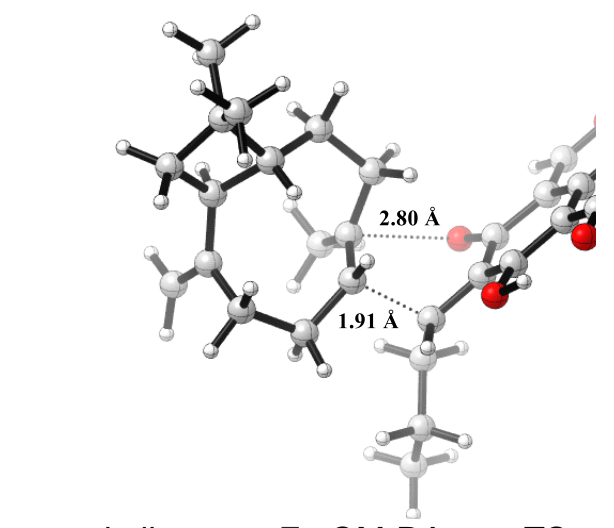

caryophyllene- $\alpha \alpha-Z-o Q M-D A-e x o-T S$

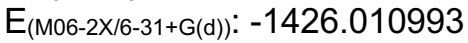

$\mathrm{TCG}_{(\mathrm{M} 06-2 \mathrm{X} / 6-31+G(\mathrm{~d})): 0.525416}$

$E_{(M 06-2 X / 6-311+G(d, p)):-1426.383138}$

$E_{(M 06-2 X / 6-311+G(d, p), s m d=H F I P):}-1426.41439$ Imaginary frequencies: 1

C $1.315607001 .42367800-2.00839200$

H $2.018375000 .85653800-2.63221300$

H $1.800133002 .35108700-1.70732500$

H $0.428607001 .63565100-2.61617400$

C $0.883440000 .57424700-0.86320500$

C 0.836545000 .985773000 .47377600

H 0.676320000 .162451001 .17327800

C $0.53270000-0.84571000-1.15293700$

$\mathrm{H}-0.16454500-1.19842800-0.38134800$

H $0.04023400-0.93505000-2.12422600$

C $1.80940100-1.73710000-1.13836600$

H $2.27833200-1.68754200-2.12891300$

H $1.47796100-2.77349100-1.00513300$

C $2.85489300-1.38589500-0.08365900$

H $2.36728600-1.140102000 .86697000$

C $3.93416100-0.32002800-0.43833500$

H $4.12171300-0.35501000-1.51952800$

C $3.99736800-2.405176000 .22535200$

C $4.97785700-1.208315000 .28911500$

H $5.14630100-0.870137001 .31791300$

H $5.94649200-1.32125300-0.20800400$

C $4.31303800-3.34830500-0.93189400$

H $5.23972000-3.89488000-0.72580600$

H $4.44664200-2.81157300-1.87791900$

H $3.51551500-4.08676700-1.07217200$

C $3.82756300-3.179742001 .52408600$

H $2.98566600-3.879362001 .46175800$

H $3.63931800-2.497851002 .36128000$

H $4.73011400-3.756477001 .75635600$

C $3.873548001 .12445700-0.00718500$

C $4.484840002 .06702000-0.72823400$

H $4.971874001 .83889600-1.67266300$

H $4.527576003 .10108100-0.39302700$ 
C 1.752463002 .051517001 .04409300

H 1.343728002 .397662002 .00003700

H 1.810029002 .924589000 .38469700

C 3.174008001 .501546001 .28251100

H 3.118855000 .642300001 .96294700

H 3.753515002 .273344001 .79935200

C - 1.910805000 .650543000 .37047900

C - 0.940288001 .670456000 .64076700

C - $2.36886800-0.197306001 .39297000$

C - $3.33021800-1.212525001 .14680400$

C $-3.79694300-1.39471200-0.18472900$

C $-3.29460100-0.63394900-1.24925200$

C - $2.279116000 .37558200-1.00943500$

C - $3.75501800-0.88082600-2.58829600$

$\mathrm{H}-3.30524900-0.25718200-3.37579200$

C -3.79533000 -2.04429400 2.22296000

H -4.54238700 -2.81013000 1.97026400

O $-4.60621100-1.72882500-2.88519100$

O -3.39911400 -1.94332100 3.39107400

O $-1.672129000 .95417600-1.93279900$

O $-1.88022100-0.032328002 .62000800$

H - $2.33330500-0.704170003 .20760400$

O $-4.70976700-2.33442800-0.39086800$

H -4.90469500 -2.33633500 -1.37223400

$\mathrm{H}-0.796725001 .799804001 .71548000$

C - $1.005685003 .00639500-0.09671200$

$\mathrm{H}-1.890338003 .00970900-0.73801600$

H - $0.159924003 .14065800-0.77865000$

C - 1.058625004 .182738000 .88123800

$\mathrm{H}-1.929856004 .066390001 .53769200$

$\mathrm{H}-0.176031004 .165001001 .53337600$

C - 1.131091005 .520099000 .15095500

$\mathrm{H}-2.013105005 .56378400-0.49556000$

$\mathrm{H}-0.248624005 .66695300-0.48172300$

$\mathrm{H}-1.185066006 .356642000 .85279400$

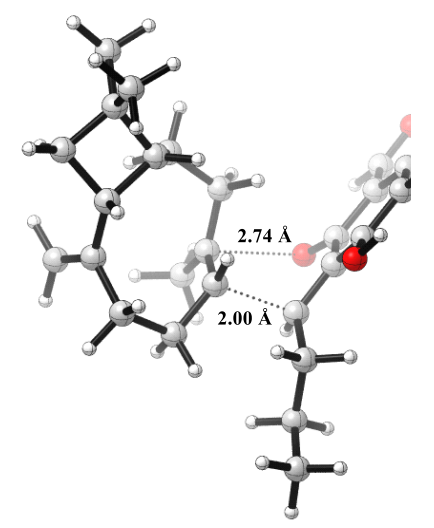

caryophyllene- $\alpha \alpha-E-o Q M-D A-e x o-T S$

$\mathrm{E}_{(M 06-2 \times / 6-31+G(d)):}-1426.005817$

TCG $(\mathrm{M} 06-2 \times / 6-31+G(d)): 0.527022$

$E_{(M 06-2 X / 6-311+G(d, p)):}-1426.377629$

$E_{(M 06-2 X / 6-311+G(d, p), \text { smd }=H F I P):}-1426.408544$

Imaginary frequencies: 1 
C $1.085628001 .06382000-2.81595500$ H $0.657472002 .06048400-2.96078000$ H $0.650086000 .39443600-3.55879400$ H $2.168675001 .13338000-2.98393100$ C $0.822984000 .55470400-1.43466200$ C $0.844861001 .38615000-0.31484200$ H 0.800404000 .866698000 .64707900 C $0.60538400-0.90435600-1.25119700$ H - $0.07774000-1.07096700-0.40441600$ H $0.14987000-1.33421700-2.14722700$ C $1.93158000-1.65067400-0.94997000$ H $2.66872300-1.42431000-1.73331400$ H $1.69101500-2.71491900-1.04866800$ C $2.51886100-1.420273000 .44005500$ H $1.69725200-1.335545001 .16512000$ C $3.63399900-0.312720000 .60255000$ H 3.598331000 .014077001 .64785700 C $3.60980200-2.467425000 .86267700$ C $4.68956300-1.427952000 .48345000$ H $5.57364300-1.346223001 .12525500$ H $5.01796000-1.57501200-0.55125500$ C $3.67869100-3.793073000 .11615100$ H $4.56255600-4.352521000 .44492900$ H $3.76128800-3.65362500-0.96663800$ H $2.79829900-4.416261000 .31596100$ C $3.56058200-2.725338002 .36830400$ H $2.67364200-3.314535002 .62916500$ H $3.52344100-1.794265002 .94436900$ H $4.44653700-3.283995002 .69198200$ C $3.731652000 .92998600-0.24804600$ C $4.375433000 .96015800-1.41975300$ H $4.845795000 .08253200-1.85375500$ H $4.463820001 .88561600-1.98654500$ C $1.759301002 .59507400-0.31268500$ H 1.356281003 .424125000 .27361600 H $1.912233002 .96423500-1.33208400$ C 3.115465002 .203551000 .31190500 H 3.810407003 .040076000 .17509600 H 2.971827002 .088134001 .39325000 C - $1.886142000 .78510000-0.08237100$ C - $1.077197001 .95047300-0.24591300$ $\mathrm{H}-1.119620002 .31059600-1.27443100$ C - 2.242875000 .227418001 .15901400 C $-3.07499300-0.918896001 .25476700$ C $-3.50557100-1.547030000 .05183300$ C $-3.09847900-1.08692000-1.20731300$ C - $2.231687000 .07500500-1.31205100$ C -3.51830700 -1.77496900 -2.39990700 $\mathrm{H}-3.15785700-1.36171800-3.35353600$ C -3.43926100 -1.45042200 2.54124900 H - $4.09061500-2.335533002 .54882400$ 
O -4.24645900 -2.77684200 -2.40038000

O -3.05791900 -0.96692300 3.61664400

O $-1.739002000 .45172700-2.39404800$

C - 1.073310003 .069315000 .78717200

$\mathrm{H}-0.256298002 .958427001 .51001800$

$\mathrm{H}-1.984055002 .975378001 .38730700$

O -1.76560800 0.787982002 .26810900

$\mathrm{H}-2.131798000 .276044003 .04587500$

O -4.29020700 -2.61203300 0.17026700

$\mathrm{H}-4.48293100-2.94472200-0.75093300$

C -1.04998500 4.45158900 0.12908800

$\mathrm{H}-1.978387004 .58191700-0.44127900$

$\mathrm{H}-0.235756004 .51391600-0.60386700$

C -0.910745005 .569661001 .15900200$

$\mathrm{H}-1.722070005 .527753001 .89379000$

H -0.935641006 .555554000 .68590600$

H 0.035377005 .481951001 .70601100

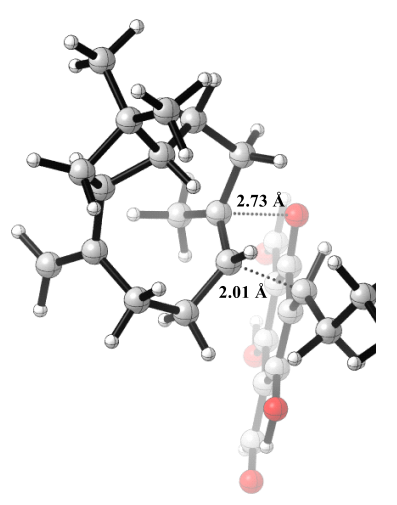

caryophyllene- $\alpha \alpha$-E-oQM-DA-endo-TS

$E_{(M 06-2 X / 6-31+G(d)):}-1426.013502$

$\mathrm{TCG}_{(\mathrm{M} 06-2 \times / 6-31+G(\mathrm{~d})): 0.526059}$

$E_{(M 06-2 X / 6-311+G(d, p)):}-1426.384883$

$E_{(M 06-2 X / 6-311+G(d, p), \text { smd=HFIP): }}-1426.415368$

Imaginary frequencies: 1

C - $2.015853000 .68175000-0.51080000$

C $-0.909213001 .31576000-1.14623000$

C - $-2.81421600-0.24024100-1.22205000$

C - $-3.94563700-0.86021700-0.63623600$

C - $4.25121400-0.564685000 .72359300$

C - 3.457187000 .298457001 .48916600

C - 2.285332000 .933179000 .90348600

C -3.78544700 0.547175002 .86867000

$\mathrm{H}-3.118686001 .232081003 .41257900$

C $-4.76374000-1.76330100-1.40288800$

$\mathrm{H}-5.63329400-2.20207500-0.89392900$

O -4.755684000 .042674003 .45037000$

O $-4.53580500-2.06133400-2.58338700$

O -1.491241001 .616110001 .57722200$

O $-2.50087100-0.50595200-2.48792900$

H -3.18180400 - $1.14830900-2.83973300$

O $-5.31896800-1.155613001 .24545500$ 
H $-5.38884200-0.859638002 .19699200$

C $0.19322100-0.779357001 .37848600$

H $0.05267500-0.439515002 .40634700$

H $0.81178100-1.685223001 .36819300$

H $-0.77985100-1.070412000 .96042900$

C 0.830355000 .275920000 .54102600

C $0.782062000 .24404800-0.84335400$

H $1.431131000 .96551900-1.34015900$

C 1.674565001 .300016001 .22072400

H 1.881425002 .133180000 .53860000

H 1.171918001 .690981002 .11114500

C 3.026166000 .639076001 .60434700

H 2.87202600 -0.04866900 2.44605700

H 3.684561001 .437660001 .96991600

C $3.70612900-0.098600000 .44964400$

H $3.464272000 .39432600-0.50296400$

C $3.55225800-1.641487000 .28621500$

H $3.54419800-2.087113001 .28932100$

C $5.25193900-0.299945000 .46925600$

C $5.01098900-1.65380600-0.24184300$

H $5.03483900-1.54047200-1.33286100$

H $5.63481400-2.507316000 .04373800$

C $5.82165900-0.495830001 .87402000$

H $6.85977200-0.841667001 .80971800$

H $5.26008000-1.237669002 .45224700$

H 5.819195000 .443074002 .43984900

C $6.057817000 .74531700-0.28756800$

H 6.000286001 .720454000 .21318400

H $5.684256000 .86712200-1.31066700$

H $7.115615000 .46208800-0.34466600$

C $2.50481700-2.35078500-0.53949100$

C $2.08880900-3.56684900-0.16782400$

H $2.42998500-4.030064000 .75536900$

H $1.39384000-4.14178100-0.77621000$

C $0.60250800-1.02890800-1.63984200$

H $0.19697500-0.79509400-2.62960900$

$\mathrm{H}-0.11039400-1.70751800-1.15793600$

C $1.96460700-1.73905600-1.81822000$

H $2.68570000-1.02616700-2.24172400$

H $1.83653500-2.53205900-2.56293500$

$\mathrm{H}-0.842082001 .06997700-2.20768200$

C - $0.547737002 .76019400-0.85506200$

$\mathrm{H}-1.502868003 .30457500-0.91374100$

$\mathrm{H}-0.218479002 .887645000 .17721000$

C $0.429552003 .39734700-1.84307700$

H $0.105324003 .19104900-2.87197600$

H $1.430280002 .95859700-1.73908100$

C $0.532519004 .90669600-1.62689600$

H $1.244598005 .36224300-2.32116400$

H $0.863500005 .13194000-0.60695300$

H - $0.439112005 .38996900-1.77426300$ 


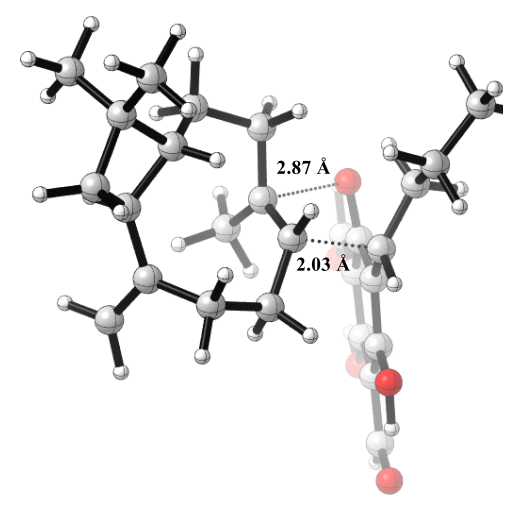

caryophyllene- $\alpha \alpha$-Z-oQM-DA-endo-TS

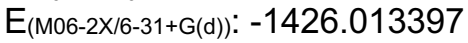
$\mathrm{TCG}_{(\mathrm{M} 06-2 \times / 6-31+\mathrm{G}(\mathrm{d}))}: 0.525987$ $E_{(M 06-2 X / 6-311+G(d, p)):}-1426.385137$ $E_{(M 06-2 X / 6-311+G(d, p), \text { smd=HFIP): }}-1426.415652$ Imaginary frequencies: 1

C $2.001176000 .59474200-0.61086900$ C $0.862389001 .32708600-1.04770400$ H $0.533271000 .97746700-2.02613100$ C 2.868687000 .985625000 .42798700 C 3.997199000 .206950000 .78819200 C $4.22578000-1.020776000 .10209800$ C $3.36610200-1.47758700-0.90463900$ C $2.21024800-0.68519300-1.29089400$ C $3.62092400-2.73501900-1.55839400$ H $2.90659800-3.03114500-2.34067200$ C 4.880921000 .644287001 .83783300 H $5.73880000-0.002440002 .06901900$ O $4.57805000-3.47289100-1.28862500$ O 4.719730001 .692562002 .47744700 O $1.38100100-1.07306000-2.13711800$ C $0.699583002 .82561100-0.87013700$ H 0.413843003 .093683000 .15153000 H $1.697284003 .26528100-1.00140400$ O 2.617750002 .121314001 .07417700 H 3.340041002 .246905001 .75437400 O $5.28786800-1.728712000 .46805500$ H $5.30306000-2.55565700-0.09107700$ C $-0.26630200-1.851288000 .06856600$ $\mathrm{H}-0.15658900-2.68187400-0.63120800$ $\mathrm{H}-0.90968000-2.153072000 .90513500$ H $0.71527000-1.619972000 .50357000$ C - $0.84277700-0.65139600-0.60330500$ C $-0.740904000 .62027100-0.05449900$ $\mathrm{H}-1.397428001 .35927200-0.51630600$ C - $1.69096000-0.84945100-1.81267900$ $\mathrm{H}-1.803325000 .10223700-2.34732900$ $\mathrm{H}-1.23456200-1.57451200-2.49269400$ C $-3.09397600-1.33833200-1.36211100$ H -3.02855800 -2.38736300 -1.04460700 H -3.74326600 -1.32131300 -2.24710700 
C -3.72628300 -0.50321800 - 0.24747800

$\mathrm{H}-3.417648000 .54723100-0.34255400$

C -3.60978500 -0.94449100 1.24365000

$\mathrm{H}-3.67645000-2.039716001 .27404500$

C $-5.27613500-0.49752000-0.07706400$

C -5.03454400 -0.36524300 1.44635900

$\mathrm{H}-4.990714000 .687487001 .75231600$

H $-5.69930700-0.908982002 .12585200$

C $-5.93189500-1.83224300-0.43024200$

$\mathrm{H}-6.97517600-1.83509700-0.09412800$

$\mathrm{H}-5.42873800-2.682649000 .04261500$

H $-5.93150900-2.00184000-1.51329900$

C $-6.005575000 .64780400-0.76343700$

$\mathrm{H}-5.948962000 .54838000-1.85514000$

$\mathrm{H}-5.569466001 .61483400-0.48809300$

$\mathrm{H}-7.066022000 .66286800-0.48478500$

C - $2.53839600-0.525995002 .22360000$

C - $2.19123400-1.362579003 .20848200$

$\mathrm{H}-2.60641200-2.365500003 .27936700$

$\mathrm{H}-1.48091700-1.071341003 .97942400$

C - 0.528987000 .846710001 .42415800

$\mathrm{H}-0.035128001 .806963001 .60088300$

H 0.117889000 .075275001 .85575300

C - 1.892897000 .845529002 .15465300

$\mathrm{H}-2.562117001 .565316001 .66265700$

$\mathrm{H}-1.734213001 .212707003 .17434500$

C - $0.261415003 .44895500-1.88310100$

$\mathrm{H}-1.257748002 .99455300-1.79714200$

H $0.091836003 .22557200-2.89762700$

C - $0.378115004 .96053300-1.69741900$

$\mathrm{H}-1.055389005 .40184400-2.43428500$

H $0.598789005 .44362500-1.80662600$

H - $0.759537005 .20463000-0.69982000$

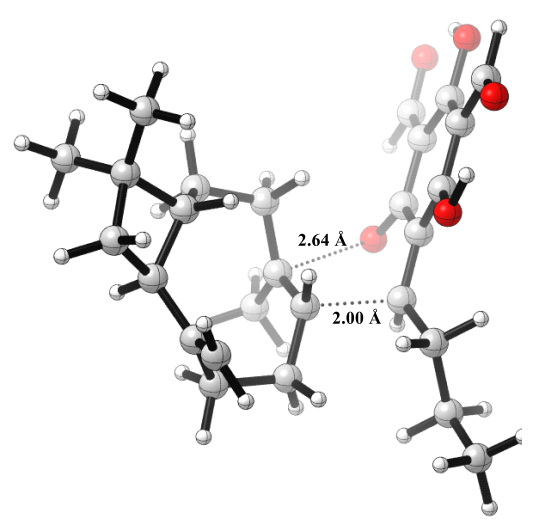

caryophyllene- $\beta \alpha$ - $E$-oQM-DA-exo-TS

$E_{(M 06-2 X / 6-31+G(d)):}-1426.009367$

TCG $($ M06-2X/6-31+G(d)): 0.52547

$E_{(M 06-2 X / 6-311+G(d, p)):}-1426.380946$

$E_{(M 06-2 X / 6-311+G(d, p), \text { smd=HFIP): }}-1426.4119$

Imaginary frequencies: 1 
C $0.916055000 .51642100-3.10911800$ H $0.31895000-0.15708700-3.72565400$ H $1.977501000 .36989300-3.35807000$ H $0.653826001 .54614400-3.36661800$ C $0.693261000 .23749500-1.65854600$ C $0.889432001 .19838400-0.66774400$ H 0.941608000 .801633000 .34835500 C $0.40404700-1.16196300-1.24603700$ $\mathrm{H}-0.17141000-1.15881300-0.30856900$ $\mathrm{H}-0.18622700-1.67453200-2.01135500$ C $1.71867600-1.94797000-1.00877900$ H $2.30682200-1.96659800-1.93654200$ H $1.41625200-2.98700300-0.82127600$ C $2.59274700-1.496893000 .15874000$ H $1.96329100-1.145154000 .98990800$ C $3.80256200-0.506853000 .00436200$ H $4.24536700-0.66949800-0.98848700$ C $3.59474800-2.546136000 .73247800$ C $4.55378400-1.373006001 .03814200$ H $4.40255100-1.011096002 .05992900$ H $5.62448700-1.531199000 .87104100$ C $4.17699700-3.47606700-0.33253800$ H $5.01785200-4.037733000 .09048500$ H $4.55122600-2.92785600-1.20439100$ H $3.43596100-4.20334100-0.68366600$ C $3.08892700-3.352085001 .91968800$ H $2.27819100-4.027479001 .61774900$ H $2.70542200-2.695205002 .70805300$ H $3.89094500-3.965483002 .34771900$ C 3.672380000 .981864000 .21872800 C 3.882996001 .569397001 .40063200 H 4.14990800 1.016352002 .29504500 H 3.798115002 .648988001 .50980800 C $1.842764002 .34869000-0.92152600$ H $1.773355003 .07702000-0.10814400$ H $1.599396002 .87570400-1.85143400$ C $3.294719001 .83364000-0.98411300$ H $3.967871002 .69627000-1.05060700$ H $3.437051001 .25377100-1.90323100$ C $-1.835692000 .86081600-0.09716900$ C $-0.946876001 .93637600-0.40151800$ $\mathrm{H}-1.056303002 .25121000-1.44033200$ C - 2.155239000 .418290001 .20213300 C -3.07285700 -0.64096700 1.42701900 C $-3.62855400-1.311886000 .30113900$ C $-3.26117900-0.97933400-1.00878600$ C $-2.311457000 .09405700-1.24416900$ C $-3.80691400-1.71022900-2.12255600$ H -3.47194900 -1.39727400 -3.12263100 C $-3.40181500-1.049329002 .76800500$ $\mathrm{H}-4.12476300-1.869965002 .87559800$ O $-4.61345000-2.64288000-2.00869500$ 
O -2.91581400 -0.53027300 3.78219000

O - $1.846709000 .34436700-2.37575100$

C -0.727579003 .089125000 .56929600$

H 0.138950002 .913773001 .21856000

$\mathrm{H}-1.581213003 .116879001 .25389100$

O -1.56459200 1.005409002 .23904200

$\mathrm{H}-1.921920000 .581025003 .07148300$

O - $4.49127800-2.292434000 .54277700$

$\mathrm{H}-4.76624900-2.67218900-0.33805000$

C - $0.623061004 .44102400-0.14093200$

$\mathrm{H}-1.573724004 .64283200-0.65079000$

H $0.141506004 .40802700-0.92515100$

C -0.310041005 .569272000 .83894100$

$\mathrm{H}-1.069398005 .626391001 .62639900$

$\mathrm{H}-0.274383006 .539302000 .33466300$

H 0.658902005 .405971001 .32516500

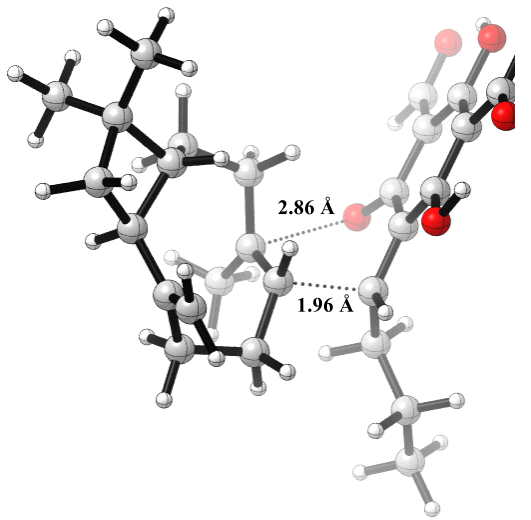

caryophyllene- $\beta \alpha$-Z-oQM-DA-exo-TS

$E_{(M 06-2 X / 6-31+G(d)):}-1426.011766$

TCG $(M 06-2 \times / 6-31+G(d)): 0.526119$

$E_{(M 06-2 X / 6-311+G(d, p)):}-1426.383722$

$E_{(M 06-2 X / 6-311+G(d, p) \text {, smd=HFIP): }-1426.415246}$

Imaginary frequencies: 1

C $0.871696001 .84001800-2.43376200$

$\mathrm{H}-0.106868001 .89833100-2.92866500$

H $1.571571001 .39326000-3.15185100$

H $1.216150002 .84598200-2.18899400$

C $0.732958000 .94585400-1.24913000$

C 0.798929001 .381882000 .06839700

H 0.888010000 .579728000 .80300300

C $0.60282200-0.51380900-1.50829700$

H $0.02872200-0.98257800-0.69614400$

H $0.07588300-0.69185100-2.45044200$

C $2.00130600-1.17621800-1.57545700$

H $2.57069500-0.74015400-2.40779500$

H $1.82393500-2.22520100-1.84846500$

C $2.84900000-1.16163400-0.30592200$

H $2.21251100-1.310928000 .57930000$

C $3.89950100-0.038144000 .02202200$

H $4.302640000 .33571000-0.92976400$ 
C $4.01424800-2.19782400-0.24248600$

C $4.82228600-1.153792000 .56011100$

H $4.69084300-1.309582001 .63507600$

H $5.89168500-1.060477000 .34320100$

C $4.66140700-2.47167300-1.60055900$

H $5.58941100-3.03688200-1.45636900$

H $4.91709400-1.54851800-2.13292400$

H $4.00826200-3.06648900-2.24933900$

C $3.68665800-3.506211000 .46165200$

H $2.96023400-4.09137800-0.11668000$

H $3.26003100-3.324093001 .45408800$

H $4.58630400-4.121000000 .58548500$

C 3.582772001 .151644000 .89869900

C 3.747273001 .142187002 .22504900

H 4.104911000 .275532002 .77069600

H 3.524654002 .025545002 .82040400

C 1.556671002 .633169000 .45704900

H 1.378599002 .843109001 .51817400

H $1.216081003 .51007900-0.10495000$

C 3.063400002 .415263000 .22630000

H 3.611333003 .286240000 .60378000

H $3.252066002 .36306800-0.85380400$

C - 1.855511000 .540583000 .36116300

C - 1.088007001 .738969000 .47844600

C - $1.99503300-0.329721001 .45960700$

C - $2.76493600-1.517590001 .38137000$

C $-3.36401700-1.851240000 .13315800$

C -3.17944500 - $1.06256400-1.01092700$

C - $2.361340000 .13849000-0.94512100$

C $-3.76545900-1.45890800-2.26426400$

$\mathrm{H}-3.57218200-0.79894500-3.12285000$

C - $-2.92301200-2.363517002 .53536400$

$\mathrm{H}-3.54455100-3.261841002 .41383900$

O $-4.45891800-2.47426600-2.41401000$

O - $2.39991600-2.129312003 .63324800$

O $-2.028472000 .78425900-1.95874900$

O $-1.38545100-0.006598002 .59834600$

$\mathrm{H}-1.60846000-0.710139003 .27135000$

O $-4.09050500-2.961829000 .08762800$

$\mathrm{H}-4.42121400-3.06454400-0.84879000$

$\mathrm{H}-0.797858001 .935300001 .51264500$

C - $1.501085003 .00511900-0.26216400$

$\mathrm{H}-2.451597002 .81929300-0.77008000$

$\mathrm{H}-0.798803003 .26515700-1.06103100$

C - 1.640441004 .186013000 .70357000

$\mathrm{H}-2.364465003 .926459001 .48668700$

$\mathrm{H}-0.684179004 .364551001 .21321800$

C - $2.088384005 .45607100-0.01549500$

$\mathrm{H}-3.052785005 .30421600-0.51187100$

$\mathrm{H}-1.362236005 .74773200-0.78306000$

$\mathrm{H}-2.195873006 .292772000 .68098300$ 


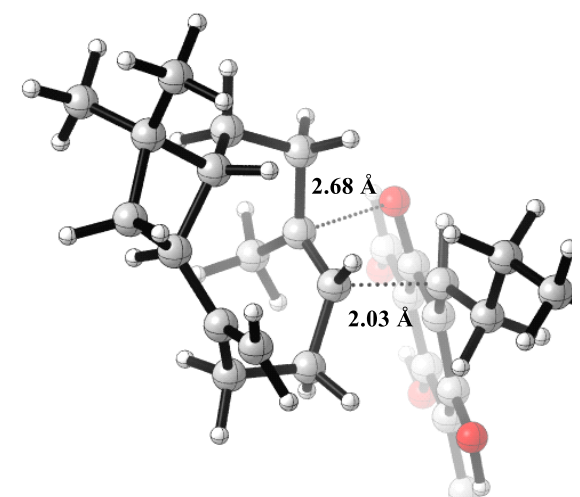

caryophyllene- $\beta \alpha$-E-oQM-DA-endo-TS

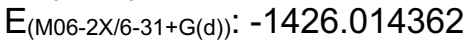

TCG $(\mathrm{M} 06-2 \mathrm{X} / 6-31+\mathrm{G}(\mathrm{d})):_{0.524671}$

$E_{(M 06-2 X / 6-311+G(d, p)):}-1426.385792$

$E_{(M 06-2 X / 6-311+G(d, p), \text { smd=HFIP): }-1426.416022}$

Imaginary frequencies: 1

C $0.13030100-1.95992600-0.68821800$

H $0.01562700-2.92585500-0.19265900$

H $0.75878500-2.09042600-1.58044000$

H - $0.85346500-1.62480100-1.04187400$

C $0.74286300-0.947230000 .22212400$

C $0.651441000 .40926600-0.03631400$

H 1.373522001 .015484000 .51006700

C $1.61307900-1.421866001 .33518900$

H $1.72918500-0.630566002 .08711100$

H $1.16018500-2.290448001 .82261700$

C $3.00538800-1.794955000 .77766400$

H $2.89749400-2.606327000 .04498700$

H $3.58441400-2.213531001 .61247600$

C $3.81766500-0.651709000 .17126100$

H 3.735812000 .244932000 .80583800

C $3.68709400-0.20252200-1.33075200$

H $3.39861700-1.07718800-1.92966900$

C $5.32950900-0.93757100-0.08824700$

C $5.22736100-0.05280700-1.35051900$

H $5.539769000 .97479800-1.14275900$

H $5.74226700-0.40042700-2.25248500$

C $5.61892400-2.39213500-0.45935300$

H $6.65138200-2.48082100-0.81675100$

H $4.96140400-2.75275500-1.25863400$

H $5.50489800-3.062104000 .40061600$

C $6.27933700-0.468851001 .00418200$

H $6.14399200-1.057675001 .92052800$

H 6.106543000 .584878001 .25050800

H 7.32434600 - 0.576979000 .68951000

C $2.838085000 .97866200-1.75524300$

C $3.224418002 .24603400-1.57442400$

H $4.166880002 .51486400-1.10661900$

H $2.593708003 .07264700-1.89571900$ 
C $0.310005000 .93696900-1.40996500$ H $0.119719002 .01276800-1.35023600$ $\mathrm{H}-0.605946000 .47761500-1.79911400$ C $1.483411000 .69294500-2.38687000$ H $1.342373001 .32675400-3.26929100$ H $1.45771800-0.34308600-2.74107200$ C -2.03988600 0.405556000 .70203200 C -0.844225000 .970459001 .21903400$ C - $2.949190001 .06753000-0.14977400$ C - $4.124276000 .43229200-0.62214900$ C $-4.36010800-0.92538000-0.25855800$ C -3.46108800 -1.64465500 0.53809300 C - $2.25692100-1.002590001 .03869200$ C - $3.72418200-3.022789000 .86279000$ $\mathrm{H}-2.97741000-3.525969001 .49452100$ C -5.04921400 1.14357400 -1.46763200 $\mathrm{H}-5.944010000 .59778700-1.79781000$ O $-4.72195000-3.645429000 .47589900$ O $-4.882945002 .31540500-1.83096100$ O $-1.39284400-1.615874001 .69582900$ O $-2.688197002 .32143600-0.50574100$ H -3.44096200 $2.63440300-1.08465600$ O $-5.46787400-1.49108200-0.72270400$ $\mathrm{H}-5.48239700-2.43387900-0.39483100$ $\mathrm{H}-0.467968000 .389397002 .06058300$ C -0.590129002 .458589001 .35418500$ $\mathrm{H}-1.534971002 .904138001 .69476800$ $\mathrm{H}-0.396933002 .931237000 .38669900$ C 0.514514002 .798431002 .35522100 H 0.264840002 .360501003 .33008700 H 1.466346002 .346132002 .04600200 C 0.701373004 .307693002 .49800900 H 1.482270004 .545533003 .22606100 $\mathrm{H}-0.225797004 .787227002 .82994500$ H 0.984954004 .758005001 .54038600

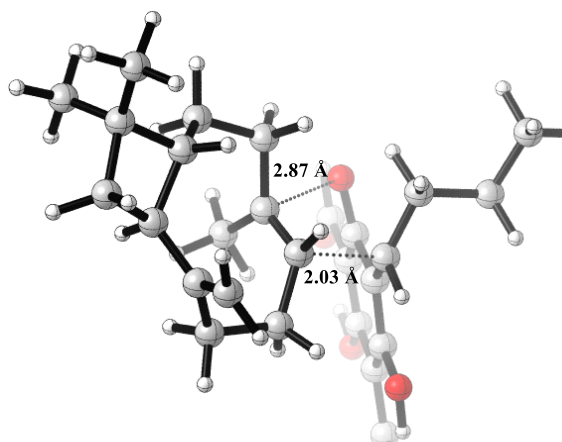

caryophyllene- $\beta \alpha$-Z-oQM-DA-endo-TS

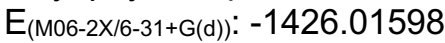
TCG $(\mathrm{M} 06-2 \mathrm{X} / 6-31+\mathrm{G}(\mathrm{d})): 0.526132$ $E_{(M 06-2 X / 6-311+G(d, p)):}-1426.387605$

$E_{(M 06-2 X / 6-311+G(d, p), \text { smd=HFIP): }-1426.417901}$ 
Imaginary frequencies: 1

C $-1.206965001 .76938400-1.93742100$

$\mathrm{H}-0.513364001 .53505800-2.75014000$

$\mathrm{H}-1.114984002 .82351000-1.67402500$

$\mathrm{H}-2.232146001 .59578300-2.29507100$

C $-0.947667000 .84412500-0.79819400$

C -0.831802001 .257751000 .53216400$

$\mathrm{H}-0.823026000 .436951001 .25368000$

C $-0.80508300-0.60678200-1.10599800$

$\mathrm{H}-0.80833600-0.75770000-2.18949500$

H $0.18243300-0.92683500-0.73267500$

C $-1.86583400-1.50571100-0.42931800$

$\mathrm{H}-1.60621900-1.657737000 .62547600$

$\mathrm{H}-1.79122600-2.49180300-0.90487300$

C $-3.30053100-0.99937700-0.52696700$

$\mathrm{H}-3.46825600-0.50345100-1.49511500$

C $-3.88122900-0.093789000 .60367400$

H -3.44363700 -0.42354700 1.55360900

C $-4.47959800-1.99020700-0.28370700$

C $-5.23337400-0.819998000 .39648700$

$\mathrm{H}-5.86588600-0.28267400-0.31768800$

H -5.82061700 -1.04462300 1.29226200

C $-4.14146400-3.093969000 .71840200$

H $-5.05147000-3.641109000 .98840600$

H $-3.70759000-2.698421001 .64324000$

H $-3.43283300-3.814686000 .29568700$

C $-5.10745500-2.58304800-1.53560400$

H $-4.40686100-3.26024500-2.03926500$

H $-5.38867000-1.79804100-2.24598800$

H $-6.00808500-3.15756100-1.29077000$

C -3.81327000 1.414368000 .53081900

C $-4.462894002 .10755200-0.40737300$

H -5.06789600 $1.62297800-1.16994700$

$\mathrm{H}-4.416644003 .19332800-0.44412200$

C - 1.553350002 .488462001 .05232700

$\mathrm{H}-1.645617003 .245553000 .26905200$

$\mathrm{H}-0.982236002 .941567001 .87150000$

C -2.96368400 2.13404300 1.56281400

$\mathrm{H}-3.454733003 .064748001 .86645100$

$\mathrm{H}-2.872128001 .515375002 .46332600$

C 1.844753000 .481674000 .35797900

C 1.037603001 .635748000 .64477800

C $2.19139100-0.400783001 .39875000$

C $3.05735300-1.506082001 .19355400$

C $3.54995000-1.74184600-0.12016100$

C $3.16301300-0.94041800-1.20321800$

C $2.246519000 .17430600-1.01259100$

C $3.64967600-1.24125600-2.52214900$

H $3.29460800-0.58394100-3.32959900$

C $3.41691300-2.357000002 .29389700$

H $4.10319600-3.187531002 .07556200$

O $4.42561600-2.17093100-2.78170800$ 
O $2.99849900-2.202052003 .44955500$

O $1.766705000 .81119100-1.97002500$

O $1.69230700-0.173436002 .61177000$

H $2.06720400-0.879044003 .21812800$

O $4.37249600-2.76778100-0.29013400$

H $4.60158900-2.79503300-1.26476200$

H 0.945046001 .782134001 .72294500

C $1.305503002 .94602600-0.07387900$

H 0.572130003 .698453000 .23884900

H $1.243126002 .81798200-1.15439000$

C 2.708684003 .452925000 .29396800

H $3.450305002 .72720900-0.05750600$

H 2.80954300 3.499860001 .38632000

C $2.991983004 .82381300-0.31434300$

H $2.929064004 .78448400-1.40673300$

H $3.993416005 .17345800-0.04720600$

H 2.27097900 5.570136000 .03706600

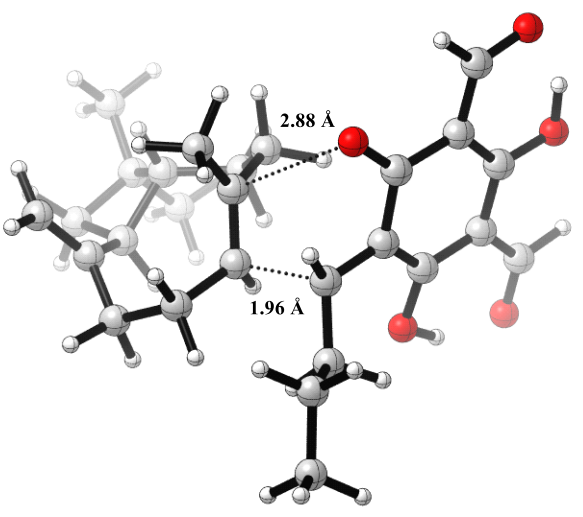

caryophyllene- $\beta \beta$ - $E$-oQM-DA-exo-TS

$E_{(M 06-2 X / 6-31+G(d)):}-1426.008401$

TCG $(\mathrm{M} 06-2 \times / 6-31+G(\mathrm{~d})): 0.525517$

$E_{(M 06-2 X / 6-311+G(d, p)):}-1426.379909$

$E_{(M 06-2 X / 6-311+G(d, p) \text {, smd=HFIP): }}-1426.411371$ Imaginary frequencies: 1

C $-1.111814000 .71539900-2.71055200$

$\mathrm{H}-2.196614000 .75281800-2.88772000$

$\mathrm{H}-0.66356400-0.02008100-3.37896600$

$\mathrm{H}-0.694519001 .69671500-2.95266700$

C - $0.869489000 .34532400-1.28350500$

C - $0.878002001 .29781100-0.25778400$

$\mathrm{H}-0.874658000 .888476000 .75573200$

C $-0.59255800-1.07859100-0.95524100$

$\mathrm{H}-0.62951300-1.68114500-1.86816700$

H $0.44827200-1.12533400-0.58383100$

C - $1.49717700-1.693773000 .13080800$

$\mathrm{H}-1.21096700-1.316062001 .12062900$

$\mathrm{H}-1.28786800-2.771479000 .14530800$

C - $2.98638900-1.46957600-0.09846400$

$\mathrm{H}-3.21868200-1.54869400-1.17235000$

C -3.67691500 -0.191527000 .46894500$

H -3.18895100 0.066888001 .41714700 
C $-4.01598700-2.333554000 .68938800$

C - $4.91559600-1.075306000 .76444300$

$\mathrm{H}-5.63126800-1.04253700-0.06416400$

$\mathrm{H}-5.44776800-0.887166001 .70306300$

C -3.51907500 -2.74867400 2.07397700

H -4.34548000 -3.17978300 2.65072400

H -3.12447000 -1.90096400 2.64523200

$\mathrm{H}-2.72892200-3.505423002 .00662900$

C $-4.57466800-3.53466900-0.05864500$

$\mathrm{H}-3.79874800-4.29552500-0.21187200$

$\mathrm{H}-4.96072200-3.24216500-1.04176100$

H -5.39347700 -4.00113000 0.50217400

C $-3.837091001 .06368100-0.35912200$

C $-4.550333001 .07901200-1.48936000$

H $-5.035892000 .18707700-1.87985700$

$\mathrm{H}-4.672461001 .99119300-2.06966200$

C - $1.733286002 .54469200-0.41668000$

$\mathrm{H}-1.799234002 .82679700-1.47222600$

$\mathrm{H}-1.306143003 .397606000 .11513600$

C -3.158106002 .324156000 .14037700$

H -3.75905600 $3.20141600-0.12378000$

$\mathrm{H}-3.104136002 .295628001 .23624000$

C $1.875166000 .70096300-0.09264800$

C $1.007487001 .83205000-0.23678600$

H $1.032455002 .19969200-1.26431400$

C 2.256402000 .145357001 .14328300

C $3.19517800-0.915411001 .23345800$

C $3.71544800-1.463001000 .02612300$

C $3.28582200-1.01777100-1.23054700$

C $2.298835000 .04672100-1.33142500$

C $3.79623800-1.63164800-2.42885300$

H $3.40851200-1.23958800-3.38049200$

C $3.57499000-1.445464002 .51546500$

H $4.30991100-2.262512002 .51974300$

O $4.62883000-2.54839900-2.43456800$

O $3.11884900-1.031972003 .59128100$

O $1.777654000 .38077400-2.41197300$

C 1.040460002 .955672000 .80218800

H 1.905278002 .798442001 .45212200

H 0.175949002 .925056001 .47575700

O 1.696315000 .624599002 .25217600

H 2.091424000 .128943003 .02710100

O $4.60428000-2.443256000 .13832400$

H $4.84962500-2.73060500-0.78585400$

C 1.165108004 .329674000 .13733500

H 2.11281400 4.36610000 -0.41472100

H $0.374578004 .46724100-0.61125200$

C 1.114592005 .461043001 .16110800

H 1.904857005 .346253001 .91087000

H 0.154347005 .467117001 .69002000

H 1.242248006 .437220000 .68439100 


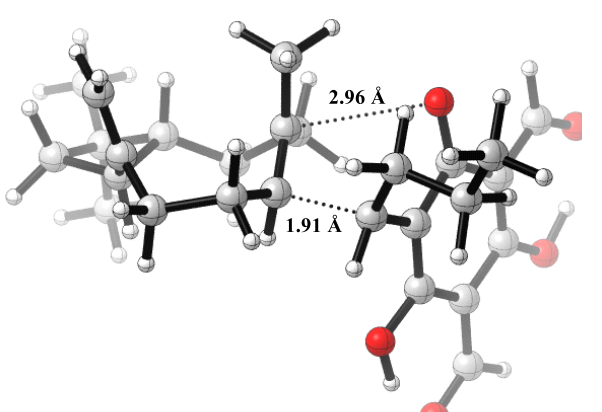

caryophyllene- $\beta \beta$-Z-oQM-DA-exo-TS

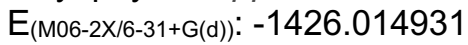

TCG $\left(M_{06}-2 X / 6-31+G(d)\right): 0.523749$

$E_{(M 06-2 X / 6-311+G(d, p)):}-1426.38694$

$E_{(M 06-2 X / 6-311+G(d, p), \text { smd=HFIP): }-1426.418456}$

Imaginary frequencies: 1

C -1.20696500 $1.76938400-1.93742100$

$\mathrm{H}-0.513364001 .53505800-2.75014000$

$\mathrm{H}-1.114984002 .82351000-1.67402500$

$\mathrm{H}-2.232146001 .59578300-2.29507100$

C - $0.947667000 .84412500-0.79819400$

C - 0.831802001 .257751000 .53216400

$\mathrm{H}-0.823026000 .436951001 .25368000$

C $-0.80508300-0.60678200-1.10599800$

$\mathrm{H}-0.80833600-0.75770000-2.18949500$

H $0.18243300-0.92683500-0.73267500$

C $-1.86583400-1.50571100-0.42931800$

$\mathrm{H}-1.60621900-1.657737000 .62547600$

$\mathrm{H}-1.79122600-2.49180300-0.90487300$

C $-3.30053100-0.99937700-0.52696700$

$\mathrm{H}-3.46825600-0.50345100-1.49511500$

C $-3.88122900-0.093789000 .60367400$

$\mathrm{H}-3.44363700-0.423547001 .55360900$

C -4.47959800 -1.99020700 -0.28370700

C $-5.23337400-0.819998000 .39648700$

$\mathrm{H}-5.86588600-0.28267400-0.31768800$

H $-5.82061700-1.044623001 .29226200$

C $-4.14146400-3.093969000 .71840200$

$\mathrm{H}-5.05147000-3.641109000 .98840600$

$\mathrm{H}-3.70759000-2.698421001 .64324000$

$\mathrm{H}-3.43283300-3.814686000 .29568700$

C $-5.10745500-2.58304800-1.53560400$

$\mathrm{H}-4.40686100-3.26024500-2.03926500$

H $-5.38867000-1.79804100-2.24598800$

$\mathrm{H}-6.00808500-3.15756100-1.29077000$

C -3.813270001 .414368000 .53081900$

C -4.46289400 $2.10755200-0.40737300$

H $-5.067896001 .62297800-1.16994700$

$\mathrm{H}-4.416644003 .19332800-0.44412200$

C - 1.553350002 .488462001 .05232700

$\mathrm{H}-1.645617003 .245553000 .26905200$

H -0.982236002 .941567001 .87150000$ 
C - 2.963684002 .134043001 .56281400

H -3.45473300 3.064748001 .86645100

$\mathrm{H}-2.872128001 .515375002 .46332600$

C 1.844753000 .481674000 .35797900

C 1.037603001 .635748000 .64477800

C $2.19139100-0.400783001 .39875000$

C $3.05735300-1.506082001 .19355400$

C $3.54995000-1.74184600-0.12016100$

C $3.16301300-0.94041800-1.20321800$

C $2.246519000 .17430600-1.01259100$

C $3.64967600-1.24125600-2.52214900$

H $3.29460800-0.58394100-3.32959900$

C $3.41691300-2.357000002 .29389700$

H $4.10319600-3.187531002 .07556200$

O $4.42561600-2.17093100-2.78170800$

O $2.99849900-2.202052003 .44955500$

O $1.766705000 .81119100-1.97002500$

O $1.69230700-0.173436002 .61177000$

H $2.06720400-0.879044003 .21812800$

O $4.37249600-2.76778100-0.29013400$

H $4.60158900-2.79503300-1.26476200$

H 0.945046001 .782134001 .72294500

C $1.305503002 .94602600-0.07387900$

H 0.572130003 .698453000 .23884900

H $1.243126002 .81798200-1.15439000$

C 2.708684003 .452925000 .29396800

H $3.450305002 .72720900-0.05750600$

H 2.809543003 .499860001 .38632000

C $2.991983004 .82381300-0.31434300$

H $2.929064004 .78448400-1.40673300$

H $3.993416005 .17345800-0.04720600$

H 2.27097900 5.570136000 .03706600

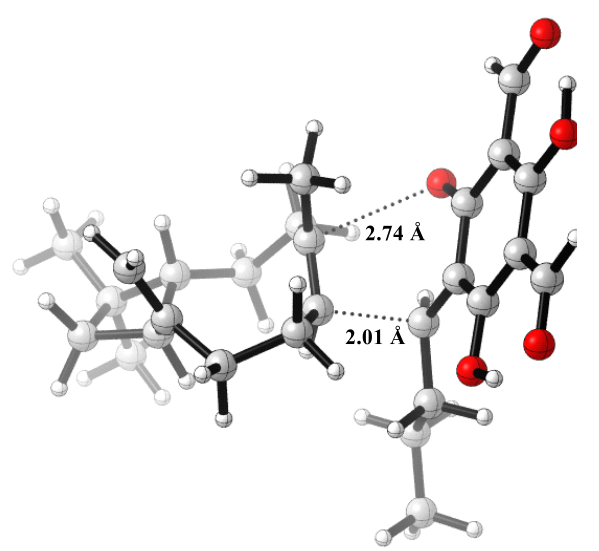

caryophyllene- $\beta \beta$ - $E$-oQM-DA-endo-TS

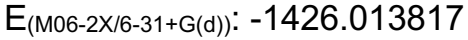

TCG $\left(\mathrm{M}_{\mathrm{M} 6-2 \times / 6-31+G(d)): 0.525369}\right.$

$E_{(M 06-2 X / 6-311+G(d, p)):}-1426.385293$

$E_{(M 06-2 X / 6-311+G(d, p), \text { smd=HFIP): }}-1426.414933$

Imaginary frequencies: 1 
C $0.11617700-1.962847000 .55061200$ H $0.71237600-2.153636001 .45540500$ H $0.00756000-2.88865500-0.01552100$ $\mathrm{H}-0.87594700-1.634767000 .88720900$ C $0.79235900-0.90605900-0.25615100$ C 0.702116000 .431899000 .10527800 H $1.423713001 .08724000-0.38275900$ C $1.63253300-1.31810300-1.43091000$ H $1.68500700-2.41157600-1.45673200$ H $1.10597000-1.02397300-2.35085000$ C $3.05623000-0.73395400-1.41622500$ H $3.039523000 .32957800-1.68967400$ H $3.62247100-1.23750200-2.21151900$ C $3.79801400-0.91151100-0.09283900$ H $3.55515900-1.894213000 .34180200$ C 3.704338000 .164071001 .03828800 H 3.608057001 .151294000 .56809000 C $5.34680500-0.73966100-0.07680100$ C $5.20135000-0.111408001 .33004900$ H $5.31888500-0.860812002 .11994800$ H 5.829325000 .754597001 .56565500 C $5.849285000 .28500200-1.09383300$ H $6.905986000 .50709400-0.90507900$ H $5.297833001 .23040900-1.03631400$ H $5.76579300-0.09192000-2.11982900$ C $6.15152800-2.02689300-0.17654600$ H $6.03753700-2.48358000-1.16807900$ H $5.82167800-2.756534000 .57166100$ H $7.21981600-1.83706500-0.01709800$ C 2.701838000 .055258002 .16894700 C $2.71630000-0.971289003 .02479000$ H $3.43511200-1.784058002 .94400100$ H $2.00091500-1.036221003 .84179200$ C 0.360192000 .842819001 .52220300 $\mathrm{H}-0.182775000 .041347002 .03095000$ $\mathrm{H}-0.291524001 .722235001 .54308800$ C 1.657361001 .149312002 .30233500 H 1.402438001 .298551003 .35704000 H 2.074551002 .097580001 .93765100 C - $1.998112000 .46571500-0.63485800$ C - $0.791298001 .06787500-1.08470500$ C - -2.921841001 .058320000 .25169500$ C -4.118572000 .400755000 .62942200$ C - $4.36799000-0.908330000 .12366300$ C - $3.46435000-1.55770700-0.72556600$ C - $-2.23579500-0.89338200-1.12880000$ C $-3.74526800-2.88848600-1.19925400$ $\mathrm{H}-2.99345700-3.33828500-1.86420300$ C -5.053364001 .040979001 .51888100$ $\mathrm{H}-5.963018000 .480159001 .77458200$ O $-4.76353800-3.52675200-0.90122200$ O -4.880230002 .169823001 .99795800$ 
O - $1.37485300-1.44895700-1.83805400$

O -2.65824800 2.27107700 0.73069600

$\mathrm{H}-3.423507002 .536218001 .31854000$

O $-5.49532700-1.497416000 .50355200$

$\mathrm{H}-5.51881400-2.400373000 .07786900$

$\mathrm{H}-0.416639000 .56734600-1.97808100$

C - $0.541294002 .56494200-1.09379600$

$\mathrm{H}-0.295765002 .94529300-0.09639900$

$\mathrm{H}-1.495366003 .04571400-1.34621000$

C $0.519853002 .98720300-2.11066600$

H $0.206098002 .66462100-3.11138200$

H $1.471080002 .47614400-1.90765800$

C $0.745706004 .49769200-2.10088200$

H $1.492971004 .79422900-2.84256500$

H $1.092957004 .83543500-1.11816300$

H - $0.183034005 .03260700-2.32661000$

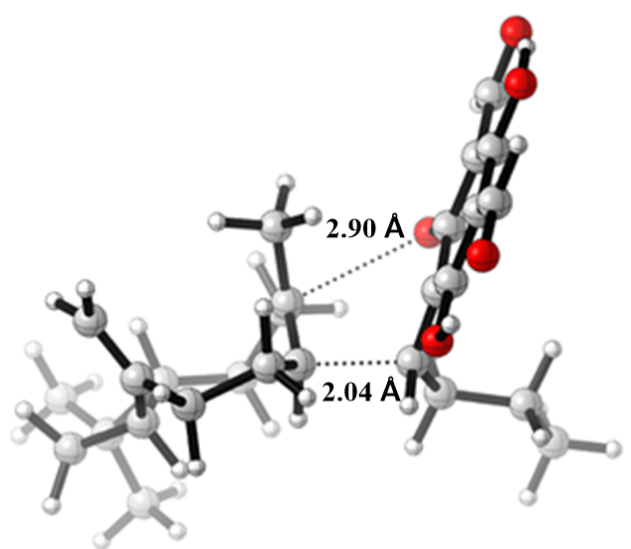

caryophyllene- $\beta \beta$-Z-oQM-DA-endo-TS

$E_{(M 06-2 X / 6-31+G(d)):}-1426.013723$

$\mathrm{TCG}_{(\mathrm{M} 06-2 \times / 6-31+\mathrm{G}(\mathrm{d}))}: 0.525687$

$E_{(M 06-2 X / 6-311+G(d, p)):}-1426.385407$

$E_{(M 06-2 X / 6-311+G(d, p), \text { smd=HFIP): }-1426.414787}$

Imaginary frequencies: 1

C - $-0.14447900-0.690844001 .69600800$

$\mathrm{H}-0.72088900-1.586959001 .96752300$

H $0.02285800-0.086989002 .58906600$

H $0.82368500-1.038970001 .31307200$

C -0.896722000 .071276000 .65748400$

C - $0.88072800-0.34841400-0.66454400$

$\mathrm{H}-1.568706000 .16471500-1.33692100$

C - 1.699783001 .257227001 .11542800

$\mathrm{H}-1.816328001 .177394002 .20313100$

$\mathrm{H}-1.101306002 .164300000 .95775100$

C - 3.088883001 .388522000 .47786600

$\mathrm{H}-3.010332001 .63005000-0.59067300$

$\mathrm{H}-3.583167002 .251264000 .94527900$

C -3.96228200 0.153119000 .67980400

$\mathrm{H}-3.80805900-0.236995001 .69827400$

C -3.91078200 $-1.04866800-0.31207500$ 
H - $3.74750100-0.65755400-1.32392600$

C -5.49639000 0.24837000 0.42954200

C - $5.43558700-1.21210300-0.08239200$

$\mathrm{H}-5.63670500-1.928503000 .72164800$

H $-6.04697700-1.47138500-0.95343700$

C -5.86114800 $1.22075900-0.69182800$

H -6.91912800 1.10686100 -0.95499600

H -5.27376100 $1.04521000-1.60014700$

$\mathrm{H}-5.703447002 .26148400-0.38511100$

C -6.350331000 .511716001 .66079700$

$\mathrm{H}-6.179417001 .524981002 .04642600$

$\mathrm{H}-6.11647800-0.198947002 .46155800$

$\mathrm{H}-7.417736000 .419416001 .42672600$

C - $2.99066000-2.22972100-0.08423600$

C -3.05039000 -2.96551600 1.02980100

$\mathrm{H}-3.75072300-2.743746001 .83269000$

$\mathrm{H}-2.39749700-3.822908001 .17779600$

C $-0.64011700-1.80254900-1.02565300$

$\mathrm{H}-0.03631300-2.28919500-0.25412100$

$\mathrm{H}-0.08100100-1.89758400-1.96101700$

C - $1.98748900-2.55044100-1.17518100$

$\mathrm{H}-1.78106900-3.62588800-1.19436200$

H - $2.42572000-2.29456700-2.14835300$

C $1.925751000 .29659500-0.60051500$

C $0.768360000 .62561800-1.35853100$

C $2.77581700-0.75165300-1.02455600$

C $3.96198800-1.08444800-0.32689800$

C $4.27953600-0.355491000 .85600200$

C 3.444586000 .654927001 .34845000

C 2.210766000 .993713000 .65386400

C 3.789564001 .346799002 .56321300

H 3.087867002 .124604002 .89785200

C $4.82509000-2.13135000-0.80899900$

H $5.73592500-2.33358400-0.22845600$

O 4.810642001 .113990003 .22444600

O $4.59084100-2.79897900-1.82557900$

O 1.385204001 .798858001 .12264700

O $2.45633500-1.41912900-2.12989700$

H $3.17369200-2.09782500-2.29198700$

O $5.40031800-0.684436001 .48510800$

H $5.47360300-0.098150002 .29105900$

H $0.697174000 .04496500-2.27844900$

C $0.300219002 .05042400-1.55012500$

H $0.073673002 .53463500-0.60141100$

$\mathrm{H}-0.603492002 .05098000-2.17293300$

C $1.397029002 .86394400-2.25746500$

H $1.722081002 .34089700-3.16618700$

H $2.268975002 .92231100-1.59652000$

C $0.908911004 .26766600-2.60784300$

H $0.597900004 .80682900-1.70655500$

H $0.052362004 .22956400-3.29014600$

H $1.698150004 .85000500-3.09244300$ 


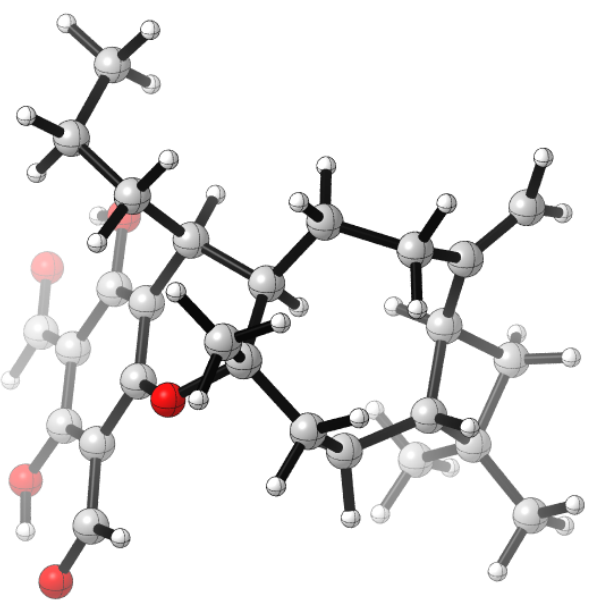

Littordial A - $\alpha$

$E_{(M 06-2 X / 6-31+G(d)):}-1426.090088$

TCG $(\mathrm{M} 06-2 \times / 6-31+G(\mathrm{~d})): 0.534693$

$E_{(M 06-2 X / 6-311+G(d, p)):}-1426.458849$

$E_{(M 06-2 X / 6-311+G(d, p), \text { smd=HFIP): }-1426.480928}$

Imaginary frequencies: none

C - $1.575896000 .56454700-0.00455600$

C - 0.729465001 .802694000 .09630000

C - $2.454767000 .29187100-1.05947800$

C - $3.24942200-0.88611500-1.08760100$

C -3.12993800 - $1.80752800-0.02788900$

C - $2.23001300-1.574096001 .03400400$

C - $1.46595500-0.376364001 .01030600$

C - $2.06915700-2.551736002 .09114500$

$\mathrm{H}-1.32391300-2.325595002 .86796400$

C $-4.16285500-1.13900700-2.18633000$

$\mathrm{H}-4.74878200-2.06813800-2.14000200$

O -2.71115200 -3.60195200 2.14990100

O $-4.30313800-0.37530400-3.14216000$

O $-0.64935500-0.166591002 .05419400$

C - 1.427879002 .888450000 .93755300

$\mathrm{H}-1.762728002 .444735001 .88303400$

$\mathrm{H}-0.708416003 .680739001 .18808800$

O - $2.533742001 .18605900-2.04473400$

$\mathrm{H}-3.193503000 .85980600-2.70868700$

O $-3.87928600-2.90834100-0.06607200$

$\mathrm{H}-3.66602100-3.453132000 .73423300$

C 0.613596000 .580823001 .93244300

C 0.666913001 .350819000 .59186600

C $1.69111600-0.514015002 .08057700$

H $2.63261400-0.053899002 .39874300$

H $1.36003200-1.123353002 .92989700$

C $1.94474900-1.418872000 .86340300$

H $2.11012100-2.441599001 .22916700$

H $1.04519400-1.478043000 .23365700$ 
C $3.16928000-1.056759000 .02206000$ H $4.02280000-0.893286000 .69923900$ C $3.196439000 .06729800-1.05728900$ H $2.217560000 .12626900-1.55109400$ C $3.62598900-2.06052100-1.07958200$ C $4.11593800-0.84535200-1.90315800$ H $5.17168300-0.62798200-1.70444800$ H $3.94986000-0.87289700-2.98543000$ C $2.45715300-2.76639200-1.76661600$ H 2.81580300 -3.30128100 - 2.65371400 H $1.68293800-2.06344500-2.09480800$ H $1.98553900-3.49945800-1.10158000$ C $4.68098700-3.07173300-0.65457200$ H $4.26858100-3.788520000 .06702400$ H $5.53791000-2.57402900-0.18729400$ H $5.04811500-3.64147600-1.51679500$ C $3.670496001 .45829400-0.70662500$ C $4.532721002 .09792700-1.50361800$ H $4.917158001 .64195200-2.41237900$ H $4.880053003 .10240900-1.27512600$ C 1.677291002 .506579000 .55849300 H $1.482235003 .08603500-0.35432300$ H 1.493380003 .190076001 .39517100 C 3.168823002 .124410000 .55861000 H 3.746984003 .043357000 .70616300 H 3.394765001 .484599001 .42003800 H 1.645422001 .925484003 .27821300 C 0.657658001 .466694003 .17454100 $\mathrm{H}-0.090508002 .260317003 .14433500$ H 0.473844000 .850449004 .05987000 H - $0.576860002 .19639700-0.91544800$ H $0.978083000 .62652200-0.16238700$ C - 2.634252003 .526527000 .23963000 $\mathrm{H}-3.141275004 .176441000 .96314000$ H -3.35659800 2.74705900 -0.03369700 C - $2.265350004 .34431100-0.99750300$ $\mathrm{H}-1.511727005 .10284700-0.75263800$ $\mathrm{H}-3.142618004 .86215100-1.39747900$ $\mathrm{H}-1.869531003 .71100800-1.79636100$ 


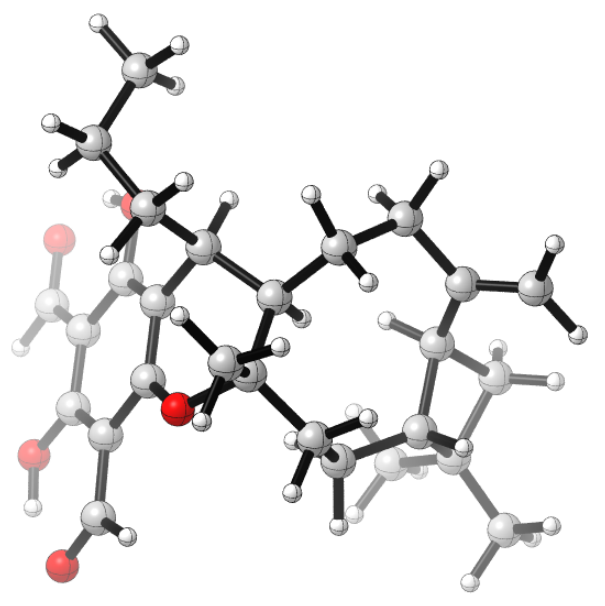

Littordial $A-\beta$

$E_{(\text {MO6-2X/6-31+G(d)): }:-1426.089845}$

TCG $(\mathrm{M} 06-2 \times 16-31+\mathrm{G}(\mathrm{d})): 0.53431$

$E_{(M 06-2 X / 6-311+G(d, p)):}-1426.45846$

$E_{(M 06-2 X / 6-311+G(d, p), \text { smd=HFIP): }-1426.480299}$

Imaginary frequencies: none

C - 1.556174000 .527150000 .04260000

C - 0.793013001 .822806000 .12497100

C - $2.439006000 .20540300-0.99547800$

C - $3.15407800-1.02198600-1.02246500$

C - $2.94805500-1.945429000 .02195900$

C - $2.03975800-1.665132001 .06482700$

C - $1.35578900-0.418715001 .04305000$

C - $1.78551100-2.646637002 .10068500$

$\mathrm{H}-1.03514800-2.385190002 .86090700$

C $-4.07141100-1.32347400-2.10528200$

$\mathrm{H}-4.59364900-2.28979000-2.05782400$

O -2.35574500 -3.73775500 2.15772900

O -4.28159500 - $0.56105200-3.04947100$

O - $0.52138500-0.190161002 .07036000$

C - 1.563596002 .860079000 .96532900

$\mathrm{H}-1.913153002 .380673001 .88730400$

$\mathrm{H}-0.891369003 .676823001 .26337600$

O -2.59547800 $1.10091300-1.97083300$

$\mathrm{H}-3.245722000 .73718300-2.62487300$

O -3.62318800 -3.09303000 -0.01403800

H -3.35332400 -3.63286200 0.77268100

C 0.630574000 .711195001 .96246900

C 0.623519001 .443950000 .61324200

C $1.86378800-0.201119002 .07967500$

H 2.75448600 0.440671002 .11651900

H $1.79878700-0.693102003 .05703700$

C $2.03059200-1.255750000 .96582700$

H 2.24306000 -2.22829500 1.43003600

H $1.08676000-1.395301000 .42005300$

C $3.16684600-0.96428600-0.00939600$

H $4.07260700-0.726583000 .56935200$

C $3.072747000 .09983900-1.15048600$ 
H $2.067694000 .08546900-1.58760600$

C $3.55840000-2.02336400-1.07832700$

C $3.97024300-0.84183900-1.99236600$

H $5.03253400-0.60285200-1.87823900$

H $3.73540500-0.91932000-3.05929100$

C $2.35402600-2.77935800-1.63903600$

H 2.65278100 -3.36064900 -2.51911600

H $1.54568000-2.10562800-1.94562200$

H $1.94470600-3.47785800-0.89937500$

C $4.65692700-2.99666300-0.67635200$

H $4.30761300-3.681644000 .10669600$

H $5.53451500-2.46348300-0.29344900$

H $4.97588400-3.60425900-1.53191700$

C $3.486609001 .53097500-0.88378100$

C $4.765822001 .86079500-0.67560300$

H $5.566165001 .12546600-0.69525000$

H $5.058354002 .88815800-0.47328900$

C 1.641831002 .595140000 .52579000

H 2.38127800 2.53800300 1.33247700

H 1.127372003 .555632000 .64985400

C $2.412307002 .60026400-0.80927700$

H $1.701599002 .47731200-1.63931400$

H $2.884006003 .58000800-0.93933200$

H 1.471126002 .194941003 .30107600

C 0.555181001 .607375003 .19627700

$\mathrm{H}-0.291429002 .292789003 .16032100$

H 0.451646000 .979875004 .08668100

$\mathrm{H}-0.675281002 .22013100-0.89104400$

H $0.939070000 .69104200-0.11185600$

C - 2.774123003 .461902000 .24226700

$\mathrm{H}-3.317531004 .090314000 .95830000$

$\mathrm{H}-3.463343002 .66065400-0.05152500$

C - $2.406448004 .29823800-0.98270500$

$\mathrm{H}-1.669037005 .06807000-0.72335300$

H -3.28844400 4.80429000 -1.38725400

$\mathrm{H}-1.991276003 .67985200-1.78359900$

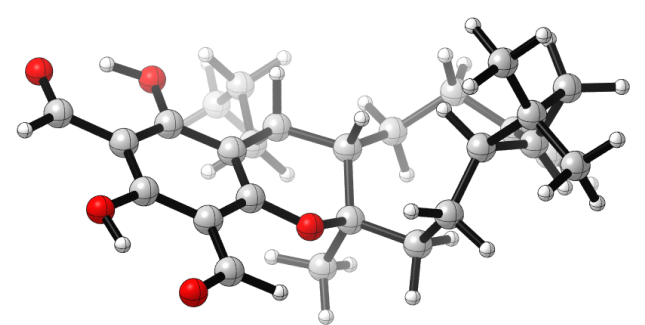

Littordial $\mathrm{B}-\alpha$

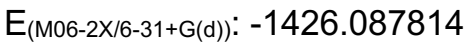

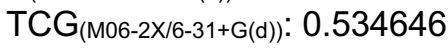

$E_{(M 06-2 X / 6-311+G(d, p)):}-1426.455989$

$E_{(M 06-2 X / 6-311+G(d, p) \text {, smd=HFIP): }-1426.477299}$

Imaginary frequencies: none

C $1.820856000 .31592200-0.10276300$ 
C $0.877822001 .49261000-0.22291700$ C $3.089217000 .35641400-0.70593300$ C $3.99157200-0.73676400-0.67243700$ C $3.59222300-1.91526700-0.01067400$ C $2.32935300-1.995568000 .60756400$ C $1.46753400-0.862167000 .54546200$ C $1.93174700-3.204758001 .30078400$ H $0.94131200-3.205587001 .77784600$ C $5.28962000-0.64962400-1.31319600$ H $5.93507600-1.53718100-1.24821900$ O $2.64276700-4.208952001 .37679300$ O $5.685904000 .35166000-1.91157800$ O $0.27783300-1.023101001 .14279000$ O $3.420464001 .48258000-1.33526300$ H $4.330011001 .36963000-1.71594700$ O $4.44317000-2.938923000 .01432500$ H $4.01519100-3.680530000 .51549200$ C -0.559778000 .137960001 .39960800$ C -0.559381001 .028223000 .13450200$ C - $1.91785400-0.480800001 .76871600$ $\mathrm{H}-1.77170300-0.967772002 .74033300$ $\mathrm{H}-2.633966000 .333843001 .93995300$ C $-2.51919100-1.522694000 .80894300$ $\mathrm{H}-1.74077800-2.227655000 .49085300$ $\mathrm{H}-3.24958800-2.104182001 .38641400$ C $-3.22941800-0.91899400-0.38887000$ $\mathrm{H}-2.50620800-0.46730600-1.07546700$ C $-4.353796000 .10297800-0.12513200$ $\mathrm{H}-4.95338200-0.230065000 .73204900$ C $-4.21336800-1.72735200-1.28832900$ C $-5.02182000-0.40850200-1.43008000$ $\mathrm{H}-4.687874000 .17087800-2.29970400$ H $-6.11505500-0.47241300-1.43927600$ C $-4.99830200-2.79736300-0.53320400$ H $-5.80372400-3.18754900-1.16631900$ $\mathrm{H}-5.45601500-2.410392000 .38388500$ $\mathrm{H}-4.35125500-3.63857600-0.25700900$ C - $3.61976800-2.30749900-2.56392500$ H - $2.92413000-3.12475900-2.33574400$ H $-3.07121600-1.54324300-3.12643400$ $\mathrm{H}-4.40491700-2.70835000-3.21638700$ C - 4.039491001 .563175000 .04453800 C -4.801921002 .338762000 .82334100$ $\mathrm{H}-5.643461001 .932444001 .37951300$ $\mathrm{H}-4.612757003 .405079000 .92804600$ C - 1.539137002 .227442000 .17431900 $\mathrm{H}-1.816818002 .473722001 .20699800$ $\mathrm{H}-0.994064003 .10055600-0.19535900$ C - $2.839544002 .15214000-0.66908300$ H -3.09479700 $3.17982700-0.94898900$ $\mathrm{H}-2.638830001 .62249900-1.60976100$ C 1.392346002 .763741000 .51745600 H 2.33895600 2.53399000 1.02342300 H 0.690085003 .058596001 .30660100 C $1.617284003 .96327300-0.40596500$ H 2.32268500 3.67576900 -1.19260600 H $0.676407004 .22413500-0.91002500$ C 2.143059005 .178874000 .35355900 H 3.099849004 .951103000 .83661500 H 1.440967005 .490119001 .13586100 
H $2.300375006 .03135400-0.31423700$ H $0.846238001 .75634000-1.29058900$ $\mathrm{H}-0.552861001 .721311002 .89849800$ C 0.023617000 .829366002 .63522300 H 1.063832001 .124614002 .47764600 $\mathrm{H}-0.008250000 .138978003 .48276700$ H - $0.851310000 .36350600-0.68629800$

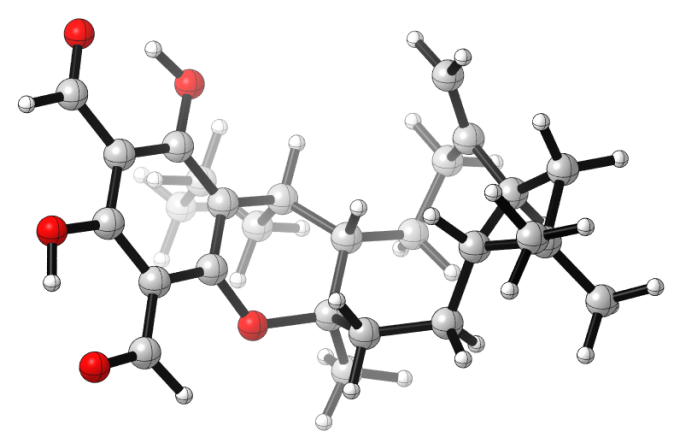

Littordial $B-\beta$

$E_{(M 06-2 X / 6-31+G(d)):}-1426.092068$

TCG $(\mathrm{M} 06-2 \times / 6-31+G(\mathrm{~d})): 0.534246$

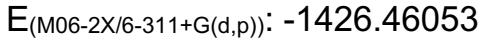

$E_{(M 06-2 X / 6-311+G(d, p), \text { smd=HFIP): }-1426.483029}$

Imaginary frequencies: none

C -1.645613000 .384141000 .08910100$

C - $0.765430001 .57917900-0.15852300$

C - 2.517797000 .251486001 .17678000

C - $3.35632800-0.886681001 .32504400$

C $-3.29586500-1.905927000 .35326100$

C - $2.41136800-1.80703100-0.74224400$

C $-1.60108500-0.64630700-0.83849500$

C $-2.31797700-2.87704300-1.71524800$

$\mathrm{H}-1.58473500-2.74887400-2.52510300$

C - $4.26128000-0.999351002 .45477700$

H -4.88125600 -1.90598100 2.50445600

O $-3.00388900-3.89953000-1.66876300$

O $-4.35870700-0.143706003 .33455200$

O $-0.80209100-0.54105900-1.91715100$

O -2.552227001 .241588002 .06702600$

$\mathrm{H}-3.212520001 .007517002 .76723300$

O $-4.08791300-2.966774000 .50587400$

H -3.91660700 -3.58830500 - 0.24671200

C $0.538030000 .04240100-1.77406600$

C $0.613948001 .02101900-0.57965500$

C $1.44661400-1.17943600-1.54922100$

H $1.00835700-1.76049400-0.72448700$

H $1.35748700-1.80205900-2.44856200$

C $2.94262300-0.96553800-1.24475600$

H $3.43149100-1.90734000-1.52852900$

H $3.38034500-0.20957900-1.91121700$

C $3.34456700-0.704487000 .21428600$

H 2.57910000 - 1.121957000 .88606000

C 3.764298000 .689959000 .80235400

H 4.296778001 .252968000 .02311900

C $4.72621100-1.255717000 .68562900$

C $4.79711100-0.063565001 .66768900$

H $4.38904900-0.337998002 .64547700$ 
H 5.769281000 .419656001 .81165000

C $5.81538900-1.15574700-0.38297900$

H $6.79660200-1.330881000 .07349800$

H $5.84186500-0.16853100-0.85788900$

H $5.67986400-1.90482600-1.17154100$

C $4.70429200-2.651841001 .28969400$

H $4.45488200-3.402961000 .52885300$

H $3.96043300-2.722656002 .09101200$

H $5.68279900-2.915657001 .70910900$

C 2.741993001 .596424001 .44437500

C 2.319978001 .449081002 .70344100

H 2.713086000 .684230003 .36674500

H 1.553450002 .100362003 .11595700

C $1.683678002 .10215900-0.78002600$

H $1.315174002 .90605400-1.42614000$

H $2.544876001 .67456700-1.30288700$

C 2.18215300 2.67989500 0.55028700

H 2.973732003 .411321000 .33469100

H 1.383462003 .221950001 .06954200

C - $1.427721002 .54960400-1.15383500$

$\mathrm{H}-1.665116002 .01708600-2.08491600$

$\mathrm{H}-0.723111003 .35077000-1.41457400$

C $-2.704858003 .18726100-0.60477300$

H -3.43907800 2.40353400 -0.38012300

$\mathrm{H}-2.482272003 .680076000 .35030700$

C - $3.310698004 .19087900-1.58374300$

$\mathrm{H}-3.561373003 .70784000-2.53501300$

$\mathrm{H}-2.608267005 .00427900-1.79871100$

H - $4.226352004 .63661600-1.18355000$

$\mathrm{H}-0.630492002 .108296000 .79321800$

H $1.844190000 .93888600-3.26314300$

C $0.790303000 .67783600-3.13730300$

H $0.192099001 .58194100-3.27669100$

H $0.52117000-0.03997700-3.91782000$

H 0.929208000 .420064000 .28327600

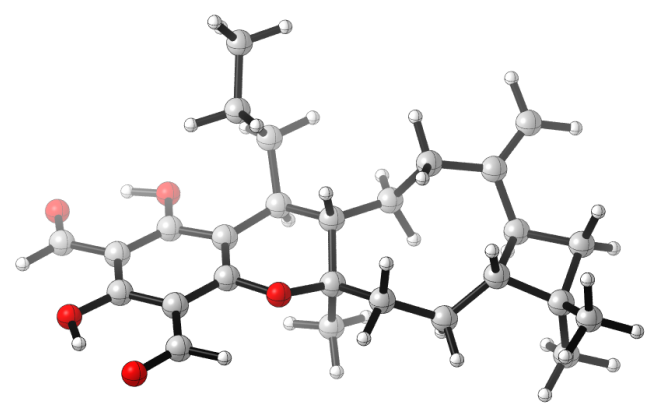

Littordial $\mathrm{F}-\alpha$

$E_{(M 06-2 X / 6-31+G(d)):-1426.089128}$

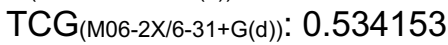

$E_{(M 06-2 X / 6-311+G(d, p)):}-1426.457787$

$E_{(M 06-2 X / 6-311+G(d, p), \text { smd=HFIP): }-1426.479869}$

Imaginary frequencies: none

C - $2.127277000 .31903900-0.40886800$

C $-0.976499001 .27877800-0.64479400$

C $-3.449944000 .65907200-0.74588100$

C $-4.55844100-0.16990500-0.43395300$

C - $4.32029800-1.396657000 .21708500$ 
C -3.00508100 -1.80309800 0.51298000 C - $1.93185400-0.933865000 .16626900$ C - $2.76081400-3.092122001 .13005300$ $\mathrm{H}-1.71299600-3.369367001 .31200900$ C -5.90904600 $0.23098400-0.77994800$ H -6.71716300 -0.45908400 -0.49849500 O -3.66011300 -3.87107400 1.45227300 O -6.17925900 1.28375100 -1.35969300 O $-0.70405400-1.416997000 .42656300$ C - $1.233995002 .68278500-0.04070900$ $\mathrm{H}-0.271085003 .210327000 .00435000$ $\mathrm{H}-1.872738003 .26286300-0.71161300$ O -3.64109700 1.81679000 -1.37486600 H -4.61355600 $1.92538600-1.54062300$ O $-5.36777300-2.160954000 .51876800$ H -5.02901600 -2.98805500 0.94938500 C $0.42649400-0.81390800-0.27019500$ C $0.322064000 .70167700-0.05050700$ C $1.62820600-1.479839000 .43522000$ H $1.74654900-1.025655001 .42719100$ H $1.28724700-2.504279000 .62110100$ C $2.99701000-1.56810300-0.28832800$ H $2.88146300-1.42318500-1.36958700$ H $3.33565100-2.60683500-0.17917400$ C $4.15006000-0.696749000 .22177700$ H $4.07406600-0.586299001 .31436900$ C $4.518239000 .68490000-0.39449900$ H $4.277926000 .66719800-1.46722400$ C $5.60562600-1.16444600-0.10341700$ C $6.007317000 .32815800-0.19871900$ H 6.377136000 .707704000 .76084000 H $6.709025000 .60711900-0.99195300$ C $5.73184700-1.85431400-1.46217500$ H $6.79100900-1.96247300-1.72342800$ H $5.25062000-1.28234300-2.26354200$ H $5.28877500-2.85680700-1.45014300$ C $6.28467100-1.994024000 .97647500$ H $5.79965300-2.973411001 .07933600$ H $6.23883300-1.489440001 .94778700$ H $7.33984200-2.168651000 .73357900$ C 3.959152001 .939385000 .22275000 C 4.704308003 .027696000 .42847800 H 5.759791003 .052216000 .16833700 H 4.282585003 .929821000 .86427900 C $1.582643001 .43525400-0.53867100$ H $2.130500000 .78837700-1.23082100$ H $1.314224002 .32198900-1.12412900$ C 2.508921001 .852112000 .61661400 H 2.16316900 2.79756800 1.05261300 H 2.43061700 1.10003000 1.41381900 $\mathrm{H}-0.839247001 .40410300-1.73029800$ C - 1.856789002 .683599001 .35667500 $\mathrm{H}-1.317690001 .992243002 .01848400$ $\mathrm{H}-2.887298002 .312763001 .30000800$ C - 1.854907004 .082695001 .97041300 $\mathrm{H}-0.831865004 .456890002 .09183000$ $\mathrm{H}-2.393483004 .789067001 .32885000$ H $1.05537600-0.69912900-2.35759600$ C $0.32647700-1.22945700-1.73935900$ H $0.50913900-2.30445400-1.83702000$ 


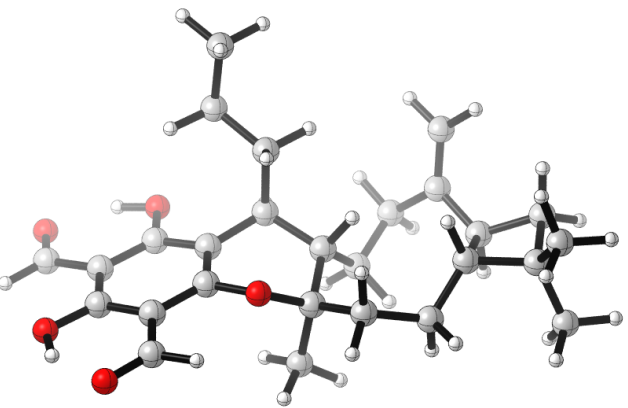

Littordial $\mathrm{F}-\beta$

$E_{(M 06-2 X / 6-31+G(d)):}-1426.091118$

$\mathrm{TCG}_{(\mathrm{M} 06-2 \times / 6-31+G(\mathrm{~d}))}: 0.533955$

$E_{(M 06-2 X / 6-311+G(d, p)):}-1426.459633$

$E_{(\text {MO6-2X/6-311+G(d,p), smd=HFIP): }}-1426.481796$

Imaginary frequencies: none

C - $1.858630000 .37162300-0.17516000$

C $-0.583295001 .17428600-0.14315700$

C -3.08955700 $0.89102100-0.60261300$

C - $4.295996000 .14930700-0.48005000$

C $-4.24680500-1.143800000 .07823800$

C -3.01606700 -1.70661700 0.47902000

C - $1.84833200-0.919823000 .32635100$

C - $2.95351500-3.061094000 .99247500$

$\mathrm{H}-1.95697200-3.448008001 .25191500$

C $-5.561614000 .71289900-0.91665500$

H -6.45491300 $0.08594900-0.78352800$

O $-3.94545500-3.775697001 .14256600$

O $-5.670131001 .83275100-1.41576500$

O $-0.66719500-1.469359000 .68172400$

C - 0.438673001 .902184001 .21126300

$\mathrm{H}-0.358774001 .144572002 .00574500$

H 0.521387002 .438927001 .19848900

O -3.09894800 2.11838100 -1.11908300

$\mathrm{H}-4.030440002 .34592400-1.36755000$

O $-5.38738200-1.822729000 .19579700$

$\mathrm{H}-5.17751700-2.710961000 .58145700$

C $0.42701300-1.22590500-0.26406300$

C $0.675519000 .29238900-0.39783800$

C $1.60148500-1.937265000 .41584300$

H $1.63544100-1.581701001 .45411800$

H $1.33660900-3.001045000 .46213000$

C $3.00804500-1.80734800-0.19882400$

H $2.97879100-1.91976400-1.29128600$

H $3.56101300-2.686174000 .15907000$

C $3.85360200-0.589315000 .19970000$

H $3.51846500-0.214218001 .17863500$

C $4.123840000 .66407100-0.70745000$

H $4.172469000 .33610800-1.75510900$

C $5.39870400-0.791809000 .28211800$

C $5.553426000 .69131200-0.12533300$

H 5.623972001 .332507000 .75872800

H $6.364474000 .94745400-0.81523800$

C $5.94728400-1.72126400-0.80111800$

H $7.03957000-1.63635800-0.84034000$ 
H $5.55951600-1.47416600-1.79576800$

H $5.70488700-2.77036400-0.59661600$

C $5.93596700-1.195584001 .64703300$

H $5.60254800-2.206677001 .91490400$

H $5.58887900-0.507751002 .42608000$

H $7.03276000-1.194104001 .65536600$

C $3.239828001 .88404500-0.62028700$

C 3.402162002 .838951000 .30023400

H 4.204129002 .818692001 .03191800

H 2.727432003 .690475000 .34603100

C $1.336788000 .65697500-1.73443600$

H $2.02411100-0.13281300-2.05298200$

H $0.571925000 .73235700-2.51796300$

C $2.127373001 .96619600-1.64313800$

H 2.56526500 2.17925400 -2.62858500

H $1.463594002 .80650500-1.40396900$

$\mathrm{H}-0.646867001 .93914600-0.92704300$

C - 1.556080002 .885394001 .54911500

$\mathrm{H}-2.513399002 .353240001 .61695600$

$\mathrm{H}-1.664374003 .609569000 .73201700$

C -1.28672400 3.615470002 .86415800

$\mathrm{H}-1.199334002 .906288003 .69513300$

$\mathrm{H}-0.351050004 .183867002 .81333400$

H $0.88524900-1.98871900-2.25530100$

C $0.03702000-1.92348200-1.56968000$

$\mathrm{H}-0.29522300-2.94209400-1.34381700$

$\mathrm{H}-0.77350000-1.40014000-2.08641500$

H 1.388084000 .544338000 .39832300

H -2.09194500 4.31619600 3.10477000

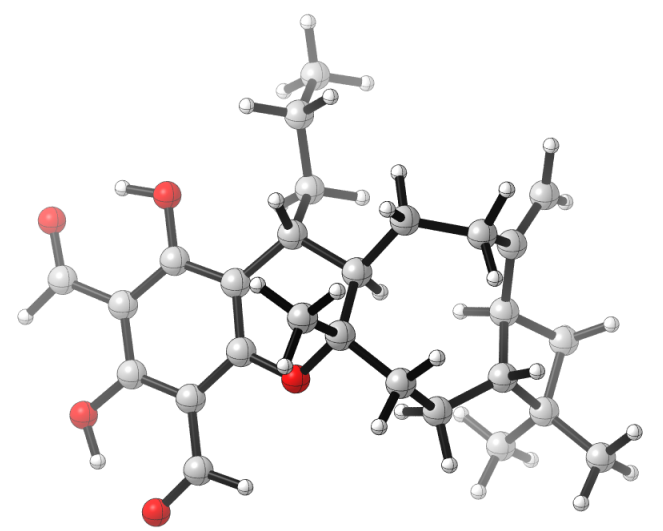

Littoridal Z $-\alpha$

$E_{(M 06-2 X / 6-31+G(d)):}-1426.055952$

TCG $(\mathrm{M} 06-2 \times / 6-31+G(\mathrm{~d})): 0.535309$

$E_{(M 06-2 X / 6-311+G(d, p))}:-1426.45999$

$E_{(M 06-2 X / 6-311+G(d, p), \text { smd=HFIP): }-1426.481311}$

Imaginary frequencies: none

C -3.13620000 $0.73850000-0.11300000$

C - $2.51980000-1.988700000 .00970000$

C -1.837600000 .371500000 .28340000$

C $-4.11970000-0.21340000-0.48680000$

C - $3.79110000-1.58160000-0.43810000$

C $-1.57890000-0.989800000 .38340000$

C $-5.439500000 .21540000-0.90570000$

H -6.14730000 - $0.57740000-1.18880000$

C - $2.20720000-3.399200000 .11500000$

$\mathrm{H}-1.21170000-3.660900000 .50240000$ 
O $-4.71370000-2.46620000-0.80290000$ $\mathrm{H}-4.31900000-3.37060000-0.68660000$ O -3.42870000 $2.03500000-0.13420000$ H - $4.371000002 .12890000-0.43860000$ O $-0.40570000-1.457200000 .83660000$ C -0.782500001 .412600000 .59640000$ $\mathrm{H}-1.098800001 .952500001 .50340000$ C 0.589400000 .751700000 .88800000 C $0.43850000-0.582700001 .63630000$ O - $2.99050000-4.29460000-0.20230000$ O $-5.790500001 .39390000-0.95590000$ C $1.72290000-1.413500001 .76640000$ H $2.41140000-0.908700002 .45100000$ H $1.41580000-2.333900002 .27660000$ C $-0.22310000-0.434900003 .00690000$ $\mathrm{H}-1.161700000 .123500002 .94510000$ H 0.441200000 .081200003 .70480000 C 3.004600001 .671500001 .51150000 H 3.422900002 .528200002 .05140000 C 3.552900001 .677400000 .10300000 C $3.500500000 .39690000-0.69580000$ H $2.602500000 .38380000-1.32760000$ C $3.63220000-0.970600000 .02310000$ C $4.723700000 .00760000-1.56040000$ H $5.648000000 .39110000-1.11530000$ H $4.700800000 .26750000-2.62340000$ C $4.47760000-1.47540000-1.18870000$ C $5.70050000-2.30810000-0.83470000$ H $6.32600000-1.79420000-0.09710000$ H $6.31350000-2.50400000-1.72200000$ H $5.40450000-3.27670000-0.41360000$ C $3.64110000-2.18240000-2.25400000$ H $2.76250000-1.59670000-2.54530000$ H $3.29270000-3.16090000-1.90490000$ H $4.24520000-2.34350000-3.15370000$ C $4.082100002 .78370000-0.42180000$ H 4.13440000 3.712500000 .14020000 H $4.483700002 .79790000-1.43190000$ C $2.42200000-1.796800000 .44670000$ H $1.67870000-1.81310000-0.35990000$ H $4.31620000-0.856000000 .87730000$ C - $0.621900002 .46560000-0.54090000$ H $0.414600002 .44060000-0.90730000$ $\mathrm{H}-1.252000002 .18910000-1.39260000$ C $-0.957100003 .89890000-0.12750000$ $\mathrm{H}-0.302300004 .207000000 .69860000$ $\mathrm{H}-1.980700003 .926700000 .25770000$ C - $0.806200004 .87550000-1.29030000$ H $0.216600004 .86470000-1.68280000$ $\mathrm{H}-1.480800004 .60750000-2.11030000$ H - $0.43980000-1.426800003 .41310000$ H 2.76940000 -2.83370000 0.54630000 C 1.474300001 .796300001 .59310000 H 1.230700002 .771100001 .15750000 H 1.175500001 .861800002 .64720000 H $1.023900000 .49840000-0.08590000$ H 3.357100000 .779900002 .03830000 H - $1.038800005 .90000000-0.98630000$ 


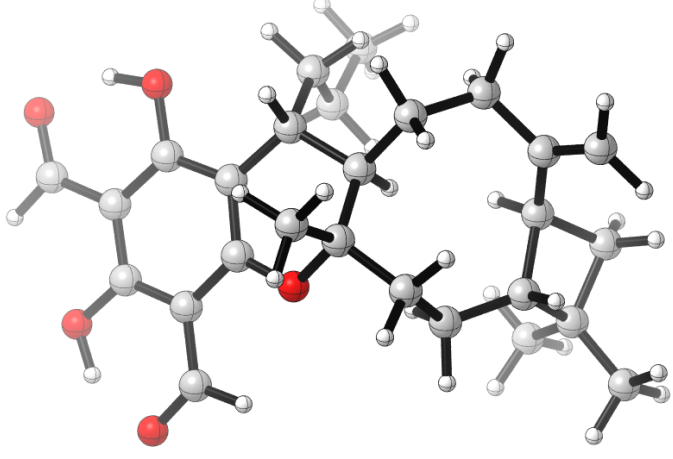

Littoridal $Z-\beta$

$E_{(M 06-2 X / 6-31+G(d)):}-1426.058801$

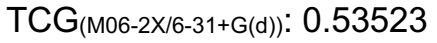

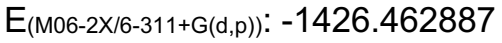

$E_{(M 06-2 X / 6-311+G(d, p), \text { smd=HFIP): }}-1426.483839$

Imaginary frequencies: none

C $3.20480000-0.926000000 .07880000$

C 2.575300001 .801800000 .12020000

C $1.89460000-0.556600000 .43990000$

C $4.187800000 .02230000-0.30840000$

C $3.852900001 .38980000-0.30150000$

C 1.632800000 .808200000 .50570000

C $5.51810000-0.40730000-0.69110000$

H $6.223400000 .38310000-0.98630000$

C 2.255100003 .213300000 .18430000

H 1.253700003 .481100000 .55110000

O $4.776100002 .26850000-0.67800000$

H $4.375900003 .17390000-0.59000000$

O $3.51700000-2.217500000 .10610000$

H $4.46620000-2.30690000-0.17960000$

O 0.458200001 .291200000 .93640000

C $0.82950000-1.585700000 .77020000$

H $1.10910000-2.067800001 .71920000$

C $-0.55270000-0.919700000 .97340000$

C -0.426900000 .430400001 .69780000$

O $3.039200004 .10320000-0.14700000$

O $5.88140000-1.58340000-0.69890000$

C - 1.737400001 .226800001 .73000000

$\mathrm{H}-2.496500000 .628100002 .24730000$

$\mathrm{H}-1.556400002 .103600002 .36110000$

C 0.159900000 .293000003 .10210000

H $1.14140000-0.189500003 .07620000$

H $-0.49850000-0.301100003 .74180000$

C $-2.66090000-2.480000000 .81230000$

$\mathrm{H}-2.25370000-2.81010000-0.15330000$

C $-3.81140000-1.527500000 .57390000$

C $-3.63150000-0.45480000-0.47110000$

$\mathrm{H}-2.74630000-0.68010000-1.07770000$

C -3.58470000 $1.03450000-0.03250000$

C $-4.78770000-0.06030000-1.42400000$

H -5.76250000 - $0.18780000-0.94200000$

$\mathrm{H}-4.80950000-0.54190000-2.40650000$

C -4.31180000 $1.41410000-1.35370000$

C - $5.389800002 .48050000-1.23540000$

H -6.09750000 2.23650000 - 0.43570000

H -5.95400000 2.57570000 - 2.17040000

H -4.94880000 $3.45930000-1.01090000$ 
C -3.34800000 $1.75070000-2.49010000$ $\mathrm{H}-2.570000000 .98900000-2.61320000$ $\mathrm{H}-2.851000002 .71150000-2.31400000$ $\mathrm{H}-3.893800001 .82350000-3.43730000$ C -4.92760000 -1.62030000 1.29930000 $\mathrm{H}-5.04830000-2.387900002 .05870000$ H -5.76190000 -0.936300001 .16480000$ C - 2.264600001 .680400000 .34980000 $\mathrm{H}-1.513500001 .48140000-0.42390000$ $\mathrm{H}-4.303100001 .155100000 .79180000$ C $0.71440000-2.71210000-0.28260000$ H $1.58120000-3.37110000-0.20750000$ $\mathrm{H}-0.15820000-3.32510000-0.02340000$ C $0.58490000-2.23800000-1.72990000$ H $1.50370000-1.71730000-2.02540000$ H - $0.22730000-1.50460000-1.82810000$ C $0.32820000-3.40510000-2.68050000$ H $1.13360000-4.14390000-2.61400000$ H - $0.60970000-3.91390000-2.43120000$ H 0.276300001 .282900003 .55140000 $\mathrm{H}-2.397600002 .769500000 .35730000$ C - $1.52430000-1.898600001 .67930000$ $\mathrm{H}-0.93870000-2.736700002 .07660000$ $\mathrm{H}-1.99080000-1.426700002 .55100000$ $\mathrm{H}-0.94590000-0.67850000-0.02130000$ $\mathrm{H}-3.04440000-3.372900001 .31760000$ H $0.26310000-3.07080000-3.71960000$

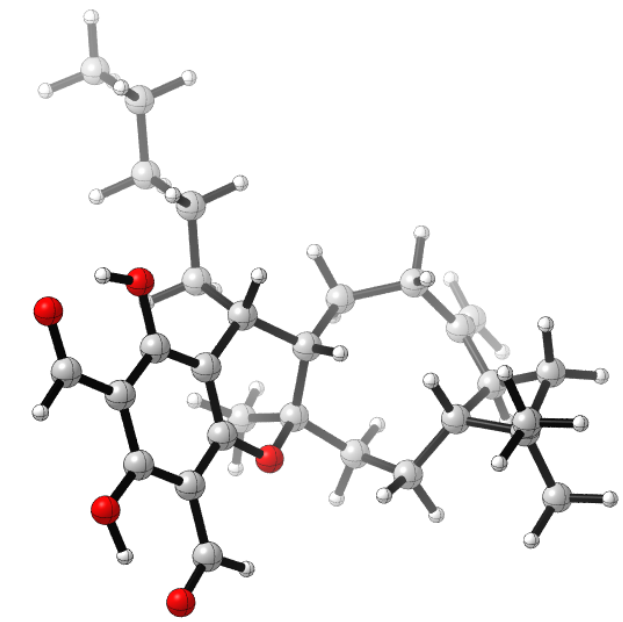

Littoridial $\mathrm{C}-\alpha$

$\mathrm{E}_{(\mathrm{MO6}-2 \times / 6-31+G(d)):}-1504.637837$

TCG $(\mathrm{M} 06-2 \times / 6-31+G(\mathrm{~d})): 0.589259$

$E_{(M 06-2 X / 6-311+G(d, p)):}-1505.062961$

$E_{(M 06-2 X / 6-311+G(d, p), \text { smd=HFIP): }-1505.086141}$ Imaginary frequencies: none

C $2.72398800-1.06764800-0.68279100$

C $1.20279800-3.036981000 .59571200$

C $1.51087700-0.68515900-0.08676700$

C $3.20187200-2.40197800-0.65850300$

C $2.42318700-3.38418600-0.01566400$

C $0.77407600-1.680597000 .54260200$

C $4.45864300-2.74625300-1.29278400$

H $4.76674300-3.80059400-1.23602200$ 
C $0.41981600-4.049935001 .27314200$

$\mathrm{H}-0.51502500-3.720446001 .74972400$

O $2.87530200-4.633971000 .00143400$

H 2.20933900 -5.18330900 0.49368500

O $3.42449200-0.11644100-1.29392100$

H $4.24412100-0.53784400-1.66709000$

O $-0.40600900-1.437499001 .13145100$

C $1.020099000 .74067400-0.20060100$

H $1.088307001 .00696300-1.26508700$

C -0.492484000 .787309000 .14640000$

$\mathrm{H}-0.987829000 .27103200-0.68317400$

C $-0.80264600-0.065790001 .39924000$

O $0.75009300-5.234432001 .33859200$

O $5.17320000-1.93100200-1.87543200$

C -0.027441000 .375554002 .64358100$

H -0.272490001 .405472002 .91844200$

H 1.051595000 .306015002 .48724200

$\mathrm{H}-0.29203200-0.274653003 .48159800$

C - $2.29192100-0.194903001 .75761600$

$\mathrm{H}-2.693249000 .810320001 .93946400$

H - $2.32319100-0.716866002 .72099800$

C -1.00472900 2.24809100 0.20174400

$\mathrm{H}-0.197624002 .88500900-0.17093500$

$\mathrm{H}-1.172381002 .565867001 .23826600$

C - $2.260869002 .62746200-0.62478300$

$\mathrm{H}-2.159713003 .68653800-0.88332600$

$\mathrm{H}-2.254919002 .08107800-1.57650700$

C -3.58272400 2.457679000 .09585900

C - $4.378085001 .20097400-0.11744100$

$\mathrm{H}-5.062760001 .069090000 .72966000$

C -3.66267500 $-0.13389200-0.40465400$

C $-5.167631000 .97427500-1.43410000$

$\mathrm{H}-4.646323001 .41893500-2.29003500$

$\mathrm{H}-6.216102001 .28699000-1.45072500$

C $-4.85764400-0.54349700-1.31793700$

C $-4.49138000-1.26872100-2.60409000$

$\mathrm{H}-3.71312500-0.72721800-3.15300700$

$\mathrm{H}-5.36241900-1.36757500-3.26196500$

$\mathrm{H}-4.11676600-2.27702900-2.39126100$

C $-5.96218700-1.29511000-0.57969700$

H -6.26297600 -0.791788000 .34534100$

$\mathrm{H}-5.64001100-2.31009700-0.32043600$

H $-6.85004900-1.37758900-1.21629500$

C -4.01814900 3.409318000 .92500000

$\mathrm{H}-3.469366004 .337157001 .06806700$

$\mathrm{H}-4.941775003 .295450001 .48618800$

C -3.19922300 -0.961913000 .77944000$

$\mathrm{H}-2.69417600-1.876876000 .44780200$

$\mathrm{H}-2.827732000 .06453600-1.08430000$

$\mathrm{H}-4.08381800-1.280715001 .34509400$

C 1.927357001 .758259000 .55483500

H 1.360061002 .265859001 .34379700 
H 2.736861001 .218417001 .06112000

C $2.549059002 .81696200-0.35749100$

H $1.755560003 .38547200-0.86436400$

H $3.120035002 .31343800-1.14573000$

C 3.453288003 .786671000 .39897700

H 4.251369003 .221611000 .90065700

H 2.879286004 .280278001 .19650400

C $4.079209004 .84929600-0.50242900$

H $3.281472005 .41241100-1.00401400$

H $4.652191004 .35436200-1.29674800$

C 4.984088005 .810454000 .26409400

H 4.423682006 .334821001 .04549000

H 5.803471005 .270352000 .75005400

H $5.423700006 .56339500-0.39623400$

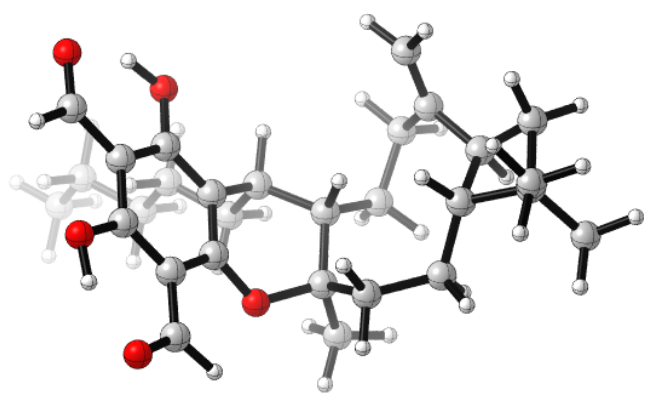

Littoridial $C-\beta$

$\mathrm{E}_{(\mathrm{M} 06-2 \times / 6-31+G(d)):}-1504.641707$

$\mathrm{TCG}_{(\mathrm{M} 06-2 \times / 6-31+\mathrm{G}(\mathrm{d}))}: 0.588419$

$E_{(M 06-2 X / 6-311+G(d, p)):}-1505.067514$

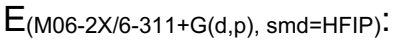

$-1505.091867$

Imaginary frequencies: none

C 2.014973000 .903285001 .29467800

C $1.447552002 .54152100-0.92309800$

C 1.280003000 .371309000 .22828900

C 2.486086002 .243781001 .27531100

C 2.200303003 .051160000 .15667100

C $1.006726001 .19653900-0.85132000$

C 3.255265002 .768408002 .38810300

H 3.590379003 .812968002 .30632700

C $1.116913003 .38959400-2.04961900$

H $0.500167002 .93875900-2.84177900$

O 2.648012004 .304014000 .15217800

H $2.347836004 .72395300-0.69560700$

O 2.278017000 .106373002 .32644800

H 2.805558000 .628983002 .98433800

O $0.341022000 .70874100-1.91582400$

C $0.78488000-1.047602000 .15931200$

H $0.74287300-1.445794001 .18064700$

C - $0.66547800-0.97600700-0.37297900$

$\mathrm{H}-1.19180800-0.369056000 .37490200$

C - $0.78679700-0.20533900-1.70766500$

O $1.481169004 .56080200-2.15041100$ 
O 3.538999002 .112065003 .38871600

C $-0.75947600-1.08255900-2.95487800$

$\mathrm{H}-1.68670600-1.65098600-3.05889400$

H $0.07630000-1.78604600-2.93225700$

H $-0.65050300-0.44205700-3.83437700$

C - $2.014096000 .72331400-1.70417000$

$\mathrm{H}-2.033791001 .21268700-2.68576300$

$\mathrm{H}-1.816636001 .51476500-0.96704600$

C $-1.37411000-2.33573800-0.41809400$

$\mathrm{H}-0.74925400-3.08792400-0.91037400$

$\mathrm{H}-2.27923900-2.25864800-1.02845300$

C $-1.78799500-2.821690000 .97583900$

$\mathrm{H}-2.32009600-3.776461000 .86489000$

$\mathrm{H}-0.90980500-3.018768001 .60056500$

C - $2.69616600-1.822940001 .65548100$

C $-3.88363500-1.367768000 .84266800$

$\mathrm{H}-4.17064700-2.170413000 .14998300$

C $-3.83464300-0.015040000 .04879500$

C $-5.15190200-0.832973001 .54055300$

H $-4.91399900-0.308175002 .47056000$

H -5.95353700 -1.55223800 1.73419600

C -5.348666000 .167776000 .37854400$

C -5.775740001 .581026000 .74350300$

$\mathrm{H}-5.149825001 .986330001 .54558200$

$\mathrm{H}-6.818376001 .603519001 .08084600$

$\mathrm{H}-5.692051002 .24976000-0.12222200$

C $-6.27327200-0.40772600-0.69398600$

$\mathrm{H}-5.98021200-1.41961100-0.99429900$

$\mathrm{H}-6.288490000 .21881300-1.59250200$

H -7.29792600 -0.45892900 -0.30948300

C - $2.42286100-1.355278002 .87400700$

$\mathrm{H}-1.53531600-1.678225003 .41108800$

$\mathrm{H}-3.06218700-0.641167003 .38377700$

C -3.40923400 $0.13423900-1.41883400$

H - $4.119561000 .84101200-1.86788200$

$\mathrm{H}-3.275686000 .701507000 .66856800$

H -3.56370800 - $0.80543900-1.96625400$

C $1.75200900-1.92378600-0.65686800$

H $1.31807400-2.92178900-0.80213300$

H $1.88751500-1.48578100-1.65466200$

C $3.11864600-2.080241000 .01012500$

H $2.98524400-2.466261001 .02973500$

H $3.59066200-1.094923000 .11819200$

C $4.05127100-3.00252900-0.77196500$

H $4.17423800-2.61665700-1.79394700$

H $3.58679600-3.99386500-0.87313400$

C $5.42533200-3.15483100-0.12240900$

H $5.30031800-3.536198000 .89906600$

H $5.88940500-2.16507200-0.02578800$

C $6.34743600-4.08191600-0.91006300$

H $5.91393300-5.08418000-0.99432100$

H $6.50646300-3.70345600-1.92542100$ 


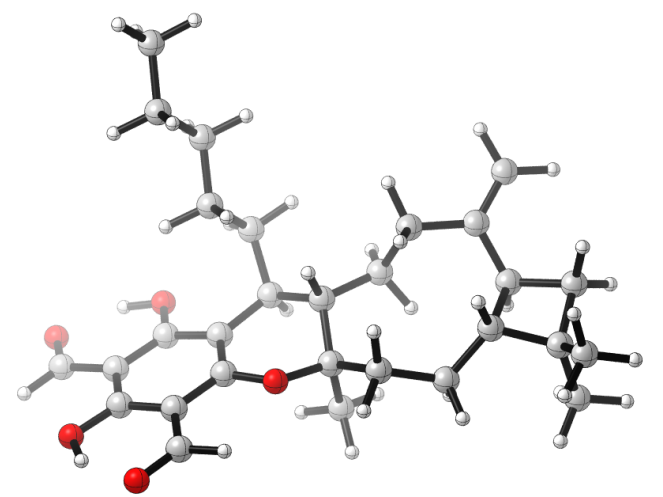

Littordial E - $\alpha$

$E_{(M 06-2 X / 6-31+G(d)):}-1504.676253$

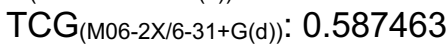

$E_{(M 06-2 X / 6-311+G(d, p)):}-1505.064759$

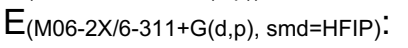

$-1505.088862$

Imaginary frequencies: none

C - $1.92325500-0.36494200-0.67953800$

C - $0.849406000 .59904800-1.14660900$

C $-3.25022400-0.26455700-1.13509300$

C $-4.29794400-1.07782000-0.63105700$

C -3.98963400 -2.04406100 0.34715800

C - $2.66204300-2.219846000 .78212500$

C - $1.65113900-1.380859000 .23283000$

C $-2.34058800-3.254387001 .74564200$

$\mathrm{H}-1.28407200-3.364896002 .02758900$

C -5.65750700 - $0.92455200-1.11326100$

H $-6.41680300-1.58460400-0.67025500$

O -3.18492100 -3.99716600 2.25035100

O $-5.98628100-0.10755000-1.97465000$

O $-0.40188700-1.647613000 .65353500$

C - $1.260140002 .08330400-0.97060200$

H $-0.350140002 .69410600-1.04846100$

$\mathrm{H}-1.908402002 .38789500-1.79586500$

O -3.50677500 0.64617300 -2.07170500

$\mathrm{H}-4.474652000 .60789300-2.28759900$

O $-4.98154000-2.792998000 .82427600$

H -4.59583200 -3.42945000 1.48034000

C $0.70802800-1.15587600-0.15510500$

C $0.460794000 .34232700-0.37883000$

C $1.92346200-1.474318000 .74289900$

H $1.94884800-0.749701001 .56665900$

H $1.66372000-2.433357001 .20446700$

C $3.33152500-1.626803000 .11113800$

H $3.26204000-1.80262700-0.96941100$

H $3.75310800-2.554574000 .51978700$

C $4.37523100-0.537865000 .38325100$

H $4.23073200-0.132624001 .39646400$

C $4.654480000 .64393100-0.59137700$

H $4.474411000 .30150700-1.62054100$

C $5.88135400-0.932030000 .24448000$

C $6.156307000 .50530500-0.26239400$

H 6.439930001 .174496000 .55813400

H $6.872500000 .61706600-1.08337800$ 
C $6.14017000-1.96077300-0.85682200$ H $7.21733100-2.03389800-1.04687000$ H $5.65578400-1.68745400-1.80106600$ H $5.78487400-2.95764900-0.57148000$ C $6.57118300-1.352276001 .53401600$ H $6.16939700-2.306344001 .89918600$ H $6.42898200-0.601853002 .31939200$ H $7.64919100-1.482234001 .37929600$ C $3.956207001 .96009600-0.37299000$ C $4.591982003 .13013500-0.46685200$ H $5.653774003 .18416200-0.69486900$ H $4.070766004 .07244300-0.31855400$ C $1.676915001 .03006600-1.02205600$ H 2.31586600 $0.27220600-1.48583800$ H $1.363648001 .68505800-1.84308700$ C $2.499945001 .84503600-0.00962200$ H 2.050478002 .836200000 .12862400 H 2.44451700 1.34440300 0.96685000 H $-0.665816000 .42943500-2.21913100$ C - 1.956621002 .408489000 .35197800 $\mathrm{H}-1.408344001 .967758001 .19745700$ $\mathrm{H}-2.955637001 .952683000 .36526600$ C - 2.093179003 .913963000 .57542800 $\mathrm{H}-1.092694004 .370508000 .60568400$ $\mathrm{H}-2.610208004 .36302300-0.28502100$ H $1.43498000-1.56978200-2.17167400$ C $0.72412700-1.97546100-1.44718400$ H $1.00666200-3.01054800-1.22969500$ H $-0.26180300-1.98012300-1.91966100$ H 0.324239000 .759937000 .63006600 C -2.846560004 .266490001 .85694700$ $\mathrm{H}-2.335172003 .808228002 .71420700$ $\mathrm{H}-3.848646003 .819228001 .82029700$ C - 2.963663005 .773908002 .07350000 $\mathrm{H}-3.507448006 .007706002 .99397900$ $\mathrm{H}-3.494793006 .247694001 .24034500$ H -1.97291300 6.23727100 2.14190500

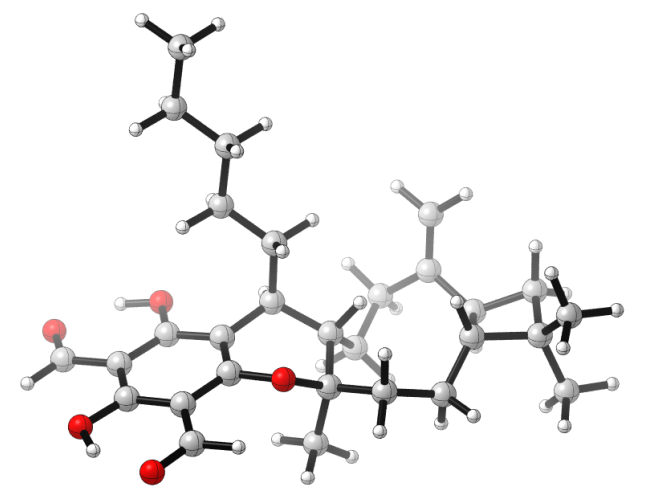

Littordial $E-\beta$

$E_{(M 06-2 X / 6-31+G(d)):}-1504.678204$

$\mathrm{TCG}_{(\mathrm{M} 06-2 \times / 6-31+G(d)): 0.587124}$

$E_{(M 06-2 X / 6-311+G(d, p)):}-1505.066487$

$E_{(M 06-2 X / 6-311+G(d, p), \text { smd=HFIP): } \quad-1505.090796}$

Imaginary frequencies: none

C - $1.858630000 .37162300-0.17516000$

C - $0.583295001 .17428600-0.14315700$

C - $-3.089557000 .89102100-0.60261300$ 
C -4.29599600 $0.14930700-0.48005000$

C $-4.24680500-1.143800000 .07823800$

C -3.01606700 -1.70661700 0.47902000

C $-1.84833200-0.919823000 .32635100$

C - $2.95351500-3.061094000 .99247500$

$\mathrm{H}-1.95697200-3.448008001 .25191500$

C $-5.561614000 .71289900-0.91665500$

H -6.45491300 $0.08594900-0.78352800$

O -3.94545500 -3.77569700 1.14256600

O -5.67013100 1.83275100 -1.41576500

O $-0.66719500-1.469359000 .68172400$

C - 0.438673001 .902184001 .21126300

$\mathrm{H}-0.358774001 .144572002 .00574500$

H 0.521387002 .438927001 .19848900

O $-3.098948002 .11838100-1.11908300$

H -4.03044000 2.34592400 -1.36755000

O $-5.38738200-1.822729000 .19579700$

H -5.17751700 -2.71096100 0.58145700

C $0.42701300-1.22590500-0.26406300$

C $0.675519000 .29238900-0.39783800$

C $1.60148500-1.937265000 .41584300$

H $1.63544100-1.581701001 .45411800$

H $1.33660900-3.001045000 .46213000$

C $3.00804500-1.80734800-0.19882400$

H $2.97879100-1.91976400-1.29128600$

H $3.56101300-2.686174000 .15907000$

C $3.85360200-0.589315000 .19970000$

H $3.51846500-0.214218001 .17863500$

C $4.123840000 .66407100-0.70745000$

H $4.172469000 .33610800-1.75510900$

C $5.39870400-0.791809000 .28211800$

C $5.553426000 .69131200-0.12533300$

H 5.623972001 .332507000 .75872800

H $6.364474000 .94745400-0.81523800$

C $5.94728400-1.72126400-0.80111800$

H $7.03957000-1.63635800-0.84034000$

H $5.55951600-1.47416600-1.79576800$

H $5.70488700-2.77036400-0.59661600$

C $5.93596700-1.195584001 .64703300$

H $5.60254800-2.206677001 .91490400$

H $5.58887900-0.507751002 .42608000$

H $7.03276000-1.194104001 .65536600$

C $3.239828001 .88404500-0.62028700$

C 3.402162002 .838951000 .30023400

H 4.204129002 .818692001 .03191800

H 2.727432003 .690475000 .34603100

C $1.336788000 .65697500-1.73443600$

H $2.02411100-0.13281300-2.05298200$

H $0.571925000 .73235700-2.51796300$

C $2.127373001 .96619600-1.64313800$

H 2.56526500 2.17925400 -2.62858500

H $1.463594002 .80650500-1.40396900$

$\mathrm{H}-0.646867001 .93914600-0.92704300$

C - 1.556080002 .885394001 .54911500

$\mathrm{H}-2.513399002 .353240001 .61695600$

$\mathrm{H}-1.664374003 .609569000 .73201700$

C - 1.286724003 .615470002 .86415800

$\mathrm{H}-1.199334002 .906288003 .69513300$

$\mathrm{H}-0.351050004 .183867002 .81333400$

H $0.88524900-1.98871900-2.25530100$ 
C $0.03702000-1.92348200-1.56968000$ H - $0.29522300-2.94209400-1.34381700$

$\mathrm{H}-0.77350000-1.40014000-2.08641500$

H 1.388084000 .544338000 .39832300

H -2.09194500 4.31619600 3.10477000 